\title{
Search for the Standard Model Higgs Boson in the Missing Energy Topology with D $\varnothing$
}

\author{
Theodoros Christoudias \\ Imperial College London
}

A thesis submitted for the degree of

Doctor of Philosophy

of the University of London

and the Diploma of Imperial College

June, 2009 


\title{
Search for the Standard Model Higgs Boson in the Missing Energy Topology with DØ
}

\author{
Theodoros Christoudias \\ Imperial College London
}

June, 2009

\begin{abstract}
A search for the Standard Model Higgs boson in the missing energy and acoplanar $b$-jet topology is reported, using an integrated luminosity of $0.93 \mathrm{fb}^{-1}$ recorded by the $\mathrm{D} \varnothing$ detector at the Fermilab Tevatron $p \bar{p}$ Collider. The analysis includes signal contributions from $p \bar{p} \rightarrow Z H \rightarrow \nu \bar{\nu} b \bar{b}$, as well as from $W H$ production in which the charged lepton from the $W$ boson decay is undetected. Neural networks are used to separate signal from background. In the absence of a signal, limits are set on $\sigma(p \bar{p} \rightarrow V H) \times B(H \rightarrow b \bar{b})$ at the 95\% C.L. of 2.6-2.3 pb, for Higgs boson masses in the range 105-135 GeV, where $V=W, Z$. The corresponding expected limits range from 2.8 to $2.0 \mathrm{pb}$. Potential improvements to the analysis with an extended dataset totalling $4 \mathrm{fb}^{-1}$ are also discussed.

Essential maintenance related to the increased luminosity and RunIIb upgrade was carried out on the impact parameter (IP) based b-tagging trigger tool and the effect of the changes on the $b$-tagger's performance was investigated.
\end{abstract}




\section{Acknowledgements}

Foremost, I would like to thank my supervisors Drs Gavin Davies and Per Jonsson for tirelessly providing me with their support and expertise. I am also sincerely thankful to Prof Peter Dornan for his guidance throughout my studies. Finally, I would like to thank everyone at the Imperial College High Energy Physics Group for their help, including Tim Scanlon and Michele Petteni.

I would like to thank the $\mathrm{D} \varnothing$ collaborators and Fermilab staff I worked with for their assistance, especially group conveners, physics coordinators and spokes for their leadership and advice. I would also like to thank the members of the $Z H \rightarrow \nu \bar{\nu} b \bar{b}$ analysis team who directly contributed to aspects of the work in my thesis.

I thank my family for providing me with support and encouragement.

I am grateful for the financial support I received from Fermilab and from the Leventis Foundation. 


\section{Contents}

Acknowledgements

List of Figures vi vi vis

List of Tables viii

Preface ix

1 Theory 1

1.1 The Standard Model . . . . . . . . . . . . . . . . . . . . . . 1

1.2 The SM Lagrangian . . . . . . . . . . . . . . . . . . . . . 3

1.2.1 Kinetic Part . . . . . . . . . . . . . 4

1.2.2 The Higgs Potential . . . . . . . . . . . . 5

1.2.3 Yukawa Interactions . . . . . . . . . . . . 6

1.3 Limits on the Higgs Boson Mass . . . . . . . . . . . . . 7

1.3.1 Theoretical Limits . . . . . . . . . . . . . . . 7

1.3.2 Experimental Limits . . . . . . . . . . . . 8

1.4 Higgs Boson Production and Decay . . . . . . . . . . . . . . . 10

1.5 ZH $\rightarrow \nu \bar{\nu} b \bar{b}$ at the Tevatron . . . . . . . . . . . 11

2 The Tevatron and the DØ Detector 14

2.1 The Tevatron .................... . . 14

2.1.1 Proton Production ................ 14

2.1.2 Anti-proton Production . . . . . . . . . . 15

2.1.3 Collisions . . . . . . . . . . . . . . . 15

2.2 The DØ Detector . . . . . . . . . . . . . . . . 17 
2.2.1 Central Tracking System . . . . . . . . . . . . . 17

2.2 .2 Calorimeter . . . . . . . . . . . . . . . . . 22

2.2 .3 The Muon Spectrometer . . . . . . . . . . . . . . . 24

2.2.4 Luminosity Monitor . . . . . . . . . . . . . . . 25

2.2 .5 The Trigger . . . . . . . . . . . . . . . 26

2.2.6 Offline Reconstruction . . . . . . . . . . . . . 30

3 Level-3 Trigger Impact Parameter $b$-tagging 31

3.1 Introduction . . . . . . . . . . . . . . . . . . . . 31

3.2 Signed Impact Parameter _ . . . . . . . . . . . . . . . 32

3.3 The Level-3 Physics Tools . . . . . . . . . . . . . . . . . . . 33

3.3.1 The Level-3 Tracker . . . . . . . . . . . . . . . . . 34

3.3.2 The Level-3 Vertex Finder . . . . . . . . . . . . . . . 36

3.3.3 The Level-3 Jet Finding Algorithm . . . . . . . . . . 37

3.4 The Level-3 IP $\boldsymbol{b}$-tagging tool . . . . . . . . . . . . . 37

3.5 Trigger Studies Samples and Tools _. . . . . . . . . . . 42

3.6 IP Significance Fit . . . . . . . . . . . . . . . . . 45

3.7 Certification . . . . . . . . . . . . . . . 45

3.8 Summary . . . . . . . . . . . . . . . . . . 48

4 Search for $Z H \rightarrow \nu \bar{\nu} b \bar{b}$ using $0.9 \mathrm{fb}^{-1}$

4.1 Introduction . . . . . . . . . . . . . . . . 51

4.2 Samples . . . . . . . . . . . . . . . . . . . 52

4.2 .1 Data . . . . . . . . . . . . . . . . . . 52

4.2.2 Monte Carlo Simulations . . . . . . . . . . . . . . . 53

4.3 MC Trigger Parametrisation . . . . . . . . . . . 56

4.4 Event Selection . . . . . . . . . . . . . . . . 57

4.4.1 Basic Selection . . . . . . . . . . . . . . 58

4.4 .2 Jets $\ldots \ldots \ldots \ldots \ldots$

4.4 .3 Leptons . . . . . . . . . . . . . . . . . . . . . 59

4.4.4 Instrumental Multijets . . . . . . . . . . . . . 60

$4.5 \quad W+$ jets Control Sample . . . . . . . . . . . . . . 61

4.6 Normalisation of the Backgrounds . . . . . . . . . . . . 63 
4.7 Taggability and $b$-tagging . . . . . . . . . . . . . . 65

4.7 .1 Introduction . . . . . . . . . . . . . 65

4.7.2 Taggability . . . . . . . . . . . . . 67

4.7.3 Operating Point Optimisation . . . . . . . . . 67

4.7 .4 b-tagging . . . . . . . . . . . . . . . . 68

4.8 Final Selection . . . . . . . . . . . . . . . . 69

4.9 Artificial Neural Network Event Classification . . . . . . . . . 72

4.9.1 Artificial Neural Networks . . . . . . . . . . . . . . 72

4.9.2 NN Variable Selection . . . . . . . . . . . . . 73

4.9.3 NN Training Procedure . . . . . . . . . . . . . . . . 74

4.9 .4 NN Results . . . . . . . . . . . . . . 80

4.10 Systematic Uncertainties . . . . . . . . . . . . . . 80

4.10 .1 Overall Uncertainties . . . . . . . . . . . . . 80

4.10.2 Differential Uncertainties . . . . . . . . . . . 85

4.11 Limits on the SM Higgs Mass . . . . . . . . . . . . . . . . . 90

5 Evolution of the $Z H \rightarrow \nu \bar{\nu} b \bar{b}$ Analysis 95

5.1 Samples . . . . . . . . . . . . . . . . . . . 96

5.1.1 Data . . . . . . . . . . . . . 96

5.1 .2 Monte Carlo Simulations . . . . . . . . . . . . . 96

5.2 Increased Signal Acceptance . . . . . . . . . . . . . . . 98

5.3 Multijet Background Treatment . . . . . . . . . . . . . 100

5.4 The $E_{T}$ Significance . . . . . . . . . . . . . . . . . . . . . . 106

5.5 Event Selection . . . . . . . . . . . . . . . . . 107

5.6 Taggability and $b$-tagging . . . . . . . . . . . . . . 110

5.7 Systematic Uncertainties . . . . . . . . . . . . . . . 117

5.8 Outlook . . . . . . . . . . . . . . . . . . . . 118

6 Conclusion $\quad 119$

$\begin{array}{lr}\text { References } & 122\end{array}$ 


\section{List of Figures}

1.1 The Higgs potential . . . . . . . . . . . . . . . . . . 5

1.2 Fit to precision electroweak observables . . . . . . . . 9

1.3 Tevatron SM Higgs combination . . . . . . . . . . . . 10

1.4 SM Higgs production and decay . . . . . . . . . . . . . . 12

1.5 Feynman diagrams for signal processes . . . . . . . . . . 13

2.1 The Fermilab accelerator complex . . . . . . . . . . . 16

2.2 Cross-sectional view of the $\mathrm{D} \emptyset$ detector . . . . . . . . . . . 18

2.3 Cross-sectional view of the central tracking system . . . . . . 19

2.4 The silicon microstrip tracker . . . . . . . . . . . . 20

2.5 The DØ Run II calorimeter . . . . . . . . . . . . . . . . 23

2.6 Trigger systems overview . . . . . . . . . . . . . . 27

3.1 L3 signed impact parameter . . . . . . . . . . . . . . 34

3.2 L3 b-tagging tools flowchart . . . . . . . . . . . 35

3.3 L3 $b$-tag online variables . . . . . . . . . . . . . . 40

3.4 L3 track parameters . . . . . . . . . . . . . . . . . . 41

3.5 IP significance fit . . . . . . . . . . . . . . 46

3.6 L3 $b$-tagging probabilities and acceptance . . . . . . . . . 49

$4.1 W+$ jets control sample . . . . . . . . . . . . . 62

$4.2 \mathcal{A}\left(\mathbb{E}_{T}, \mathscr{H}_{T}\right)$ vs. $\Delta \phi\left(\mathbb{E}_{T}, \mathbb{Z}_{T}\right) \ldots \ldots \ldots \ldots$

4.3 Normalisation of the backgrounds . . . . . . . . . . . 65

4.4 Number of jets . . . . . . . . . . . . . . 70

4.5 Dijet invariant mass . . . . . . . . . . . . 71 
$4.6 \mathrm{NN}$ input variables before $b$-tagging . . . . . . . . . . . . 75

$4.7 \mathrm{NN}$ input variables before $b$-tagging . . . . . . . . . . . . 76

4.8 NN input variables after $b$-tagging . . . . . . . . . . . . 77

$4.9 \mathrm{NN}$ input variables after $b$-tagging . . . . . . . . . . 78

$4.10 \mathrm{NN}$ training optimisation . . . . . . . . . . . . . . 81

4.11 NN output . . . . . . . . . . . . . . . . 82

$4.12 \mathrm{NN}$ output after $b$-tagging . . . . . . . . . . . . 83

4.13 Trigger uncertainty derivation . . . . . . . . . . . . 86

4.14 JES differential uncertainty . . . . . . . . . . . . . . . 87

$4.15 m_{b \bar{b}}$ differential uncertainty . . . . . . . . . . . . . . 88

$4.16 \mathrm{MC} /$ data agreement impact . . . . . . . . . . . . . 89

4.17 LLR against Higgs mass . . . . . . . . . . . . . . . . . . 93

4.18 Limits on Higgs mass . . . . . . . . . . . . . . . . . . 94

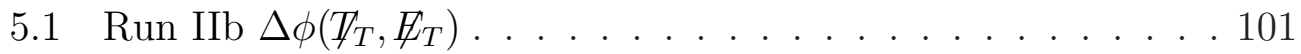

5.2 Multijet control sample before b-tagging. . . . . . . . . . . 103

5.3 Multijet control sample before $b$-tagging. . . . . . . . . . . . 104

5.4 Multijet control sample NN b-tag . . . . . . . . . . 105

$5.5 \quad E_{T}$ significance . . . . . . . . . . . . . . . 108

5.6 Signal sample before $b$-tagging . . . . . . . . . . . . . . 111

5.7 Signal sample before $b$-tagging . . . . . . . . . . . . . . . . 112

5.8 Signal sample with one tight $b$-tag . . . . . . . . . . . . . 113

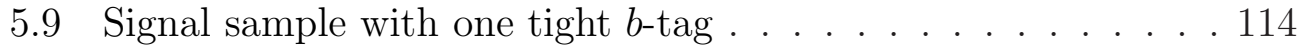

5.10 Signal sample with one tight and one loose $b$-tag . . . . . . . 115

5.11 Signal sample with one tight and one loose $b$-tag . . . . . . 116 


\section{List of Tables}

2.1 Trigger rates and latency . . . . . . . . . . . . . . 27

3.1 L3 IP $b$-tag certification samples . . . . . . . . . . . . . 47

3.2 L3 IP $b$-tag sample composition . . . . . . . . . . . . . . . 47

4.1 Run IIa luminosity trigger breakdown . . . . . . . . . . . . . . 52

4.2 Signal Monte Carlo samples . . . . . . . . . . . . . . . . 55

4.3 Background Monte Carlo samples . . . . . . . . . . . . 55

4.4 NN operating points . . . . . . . . . . . . . . 68

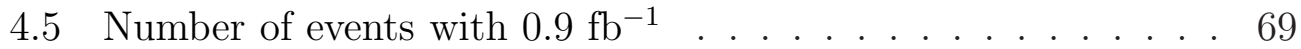

4.6 Overall systematic uncertainties . . . . . . . . . . . . . . 80

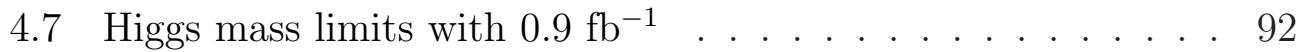

5.1 Run IIb luminosity trigger breakdown . . . . . . . . . . . . . 96

5.2 MC $K$-factors . . . . . . . . . . . . . . . . . . . . . . . . . 97

5.3 Observed/Expected number of events with $4 \mathrm{fb}^{-1}$. . . . . 117 


\section{Preface}

This thesis describes work carried out by the author between June 2006 and February 2009 as a member of the DØ collaboration. The structure of the thesis is outlined below.

Chapter 1 A brief review of the Standard Model (SM), paying particular attention to the Higgs sector.

Chapter 2 Description of the Tevatron and the D $\varnothing$ detector.

Chapter 3 The DØ Level-3 trigger impact parameter $b$-tagging tool and its certification for Run IIb.

Chapter $4 \mathrm{~A}$ search for the SM Higgs boson in the $Z H \rightarrow \nu \bar{\nu} b \bar{b}$ channel using $0.9 \mathrm{fb}^{-1}[1]$.

Chapter 5 Work in progress and the evolution of the $Z H \rightarrow \nu \bar{\nu} b \bar{b}$ analysis.

The work the author undertook as part of this thesis is outlined below, broken into Level-3 and Higgs related work.

Level-3: The author has been a member of the DØ Level-3 (L3) algorithms group since March 2006. The author's work for the L3 group centred around $b$-tagging. During this time, the online impact parameter algorithm 
was re-parametrised and re-certified a number of times to take into account changes in the tracking algorithm, the inclusion of Layer 0 in the SMT tracker and the high instantaneous luminosities of Run IIb. As part of this work, a method was developed to allow trigger development over a large sample of b-enriched data events spanning a long period of time, which has since been used in the development and certification of other tools.

Higgs: The author has been a member of the DØ Higgs group since October 2006 and has participated in two generations of the search for $Z H \rightarrow$ $\nu \bar{\nu} b \bar{b}$. The author is the main contributor to the result presented twice as preliminary [2, 3] and published with $1 \mathrm{fb}^{-1}$ in December 2008 [1]. At the time of publication, this set the most stringent limit using the missing energy and acoplanar b-jet topology at a hadron collider. Between September 2008 and February 2009, the author was one of the primary contributors in a team of analysers that has extended the $Z H \rightarrow \nu \bar{\nu} b \bar{b}$ analysis to a $4 \mathrm{fb}^{-1}$ dataset. The results of the analysis are expected to be published in 2009 . The framework developed by the team is been used for other searches by the DØ Higgs and New Phenomena groups. 


\section{Chapter 1}

\section{Theory}

This Chapter briefly covers the Standard Model (SM) of particle physics and the Higgs sector. More detail on the SM can be found in $[4,5]$. Sections 1.1 and 1.2 give a theoretical overview of the SM based on [6]. Limits currently placed on the Higgs boson mass by theory and experiment, from [7], are described in Section 1.3. The production and decay of the Higgs boson and the search strategy at hadron colliders are discussed in Section 1.4. Finally, Section 1.5 concentrates on the $Z H \rightarrow \nu \bar{\nu} b \bar{b}$ search channel at the Tevatron collider.

\subsection{The Standard Model}

The Standard Model is a powerful and far-reaching theory of elementary particles and their interactions. The elementary particles consist of quarks and leptons - collectively known as fermions, and gauge bosons, the mediators of the interactions. 
In the Standard Model there are three generations of fermions. The fermions experience two types of interactions: gauge interactions (two fermions couple to a gauge boson), and Yukawa interactions (two fermions couple to a scalar). There are twelve gauge bosons, related to the gauge symmetry

$$
G_{S M}=S U(3)_{C} \times S U(2)_{L} \times U(1)_{Y}
$$

The $S U(3)_{C}$ phase transformations operate on the colour fields, the $S U(2)_{L}$ transformations are in weak isospin $(T)$ space, and the $U(1)_{Y}$ transformations are in hypercharge $(Y)$ space $^{1}$.

Each of the three fermion generations $(i=1,2,3)$ consists of five representations of $G_{S M}$ :

$$
q_{L i}(3,2)_{+1 / 6}, u_{R i}(3,1)_{+2 / 3}, d_{R i}(3,1)_{-1 / 3}, l_{L i}(1,2)_{-1 / 2}, e_{R i}(1,1)_{-1} .
$$

The notation means that, for example, left handed quarks $q_{L}$ are triplets of $S U(3)_{C}$, doublets of $S U(2)_{L}$ and carry hypercharge $Y=+1 / 6$. When the $G_{S M}$ symmetry is manifest, all quarks, leptons and gauge bosons remain massless. However, each of the three generations of quarks and leptons have different mass scales and massive gauge bosons have been observed experimentally.

\footnotetext{
${ }^{1}$ The electric charge, $Q$, is given by $Q=T^{3}+Y / 2$ where $T^{3}$ is the eigenvalue of the third component of weak isospin.
} 
The Higgs mechanism postulates a single scalar representation

$$
\phi(1,2)_{+1 / 2}=\left(\begin{array}{c}
\phi^{+} \\
\phi^{0}
\end{array}\right)
$$

The scalar $\phi^{0}$ assumes a vacuum expectation value (vev)

$$
\left\langle\phi^{0}\right\rangle=\frac{v}{\sqrt{2}}
$$

leading to the spontaneous symmetry breaking

$$
G_{S M} \rightarrow S U(3)_{C} \times U(1)_{E M}
$$

and the generation of masses for quarks, leptons and gauge bosons. The interactions dictated by symmetry principles are best discussed within the framework of Lagrangian field theory.

\subsection{The SM Lagrangian}

The Standard Model Lagrangian, $\mathcal{L}_{S M}$, can be divided into three parts:

$$
\mathcal{L}_{S M}=\mathcal{L}_{\text {Kinetic }}+\mathcal{L}_{\text {Higgs }}+\mathcal{L}_{\text {Yukawa }}
$$

where $\mathcal{L}_{\text {Higgs }}$ and $\mathcal{L}_{\text {Yukawa }}$ are the terms describing the Higgs and Yukawa sectors respectively. 


\subsubsection{Kinetic Part}

In the kinetic part, to maintain local gauge invariance, the covariant derivative is used:

$$
D^{\mu}=\partial^{\mu}+i g_{s} G_{a}^{\mu} L_{a}+i g W_{b}^{\mu} T_{b}+i g^{\prime} B^{\mu} Y
$$

$G_{a}^{\mu}$ are the eight gluon fields, $W_{b}^{\mu}$ the three weak interaction bosons $(b=$ $1,2,3)$ and $B^{\mu}$ the single hypercharge boson. The $L_{a}$ are the $S U(3)_{C}$ generators $^{2}$ (the $3 \times 3$ Gell-Mann matrices $\frac{1}{2} \lambda_{a}$ for triplets, 0 for singlets), the $T_{b}$ are the $S U(2)_{L}$ generators (the $2 \times 2$ Pauli matrices $\frac{1}{2} \tau_{b}$ for doublets, 0 for singlets), and $Y$ is the $U(1)_{Y}$ charge.

For example, for the left handed quarks $q_{L}$ :

$$
\mathcal{L}_{\text {Kinetic }}\left(q_{L}\right)=i \overline{q_{L i}} \gamma_{\mu}\left(\partial^{\mu}+\frac{i}{2} g_{s} G_{a}^{\mu} \lambda_{a}+\frac{i}{2} g W_{b}^{\mu} \tau_{b}+\frac{i}{6} g^{\prime} B^{\mu}\right) q_{L i}
$$

where $\gamma_{\mu}$ are the Dirac matrices and $q_{i}$ denote Dirac spinors [4].

The couplings are related by $g^{\prime}=g \tan \theta_{W}$, where $\theta_{W}$ is the weak mixing angle. The physical bosons (photon $A, W^{ \pm}$and $Z^{0}$ ) exist as linear superpositions of the gauge fields, and are given by:

$$
\begin{aligned}
W_{\mu}^{ \pm} & \equiv\left(W_{\mu}^{1} \mp i W_{\mu}^{2}\right) / \sqrt{2} \\
Z_{\mu}^{0} & \equiv \cos \theta_{W} W_{\mu}^{3}-\sin \theta_{W} B_{\mu} \\
A_{\mu} & \equiv \cos \theta_{W} B_{\mu}-\sin \theta_{W} W_{\mu}^{3}
\end{aligned}
$$

\footnotetext{
${ }^{2} S U(n)$ groups have $n^{2}-1$ generators.
} 


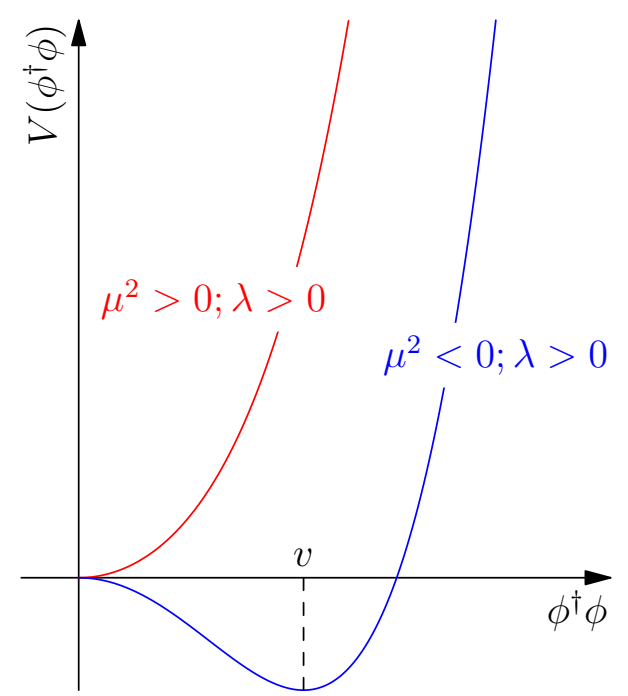

Figure 1.1: Higgs Potential $V\left(\phi^{\dagger} \phi\right)$ as a function of $\phi^{\dagger} \phi$ for $\mu^{2}>0$ and $\mu^{2}<0$. The vacuum expectation value (vev) is denoted as $v$.

\subsubsection{The Higgs Potential}

The Higgs potential, which describes the self interactions of the scalar field, is given by:

$$
\mathcal{L}_{\text {Higgs }}=\mu^{2} \phi^{\dagger} \phi-\lambda\left(\phi^{\dagger} \phi\right)^{2}
$$

The Higgs potential is plotted in Fig. 1.1 for positive and negative values of $\mu^{2}$. The parameter $\lambda$ is a coupling constant that has to be positive since the potential may not be arbitrarily negative for large $\phi$. When $\mu^{2}>0$, there is a trivial minimum at the centre of the potential $\phi=0$. The more interesting case is that for $\mu^{2}<0$, where there is an unstable maximum at $\phi=0$ and a minimum at a finite value of $|\phi|$ where $\phi^{\dagger} \phi=-\frac{-\mu^{2}}{2 \lambda}$.

$\mathcal{L}_{\text {Higgs }}$ gives interaction terms of the scalar (Higgs), $W$ and $Z$ bosons, as well as mass terms for the bosons other than the photon: 


$$
\begin{aligned}
m_{W} & =\frac{g v}{2} \\
m_{Z} & =\frac{g v}{2 \cos \theta_{W}} \\
m_{H} & =v \sqrt{2 \lambda} .
\end{aligned}
$$

The couplings of the Higgs to the gauge bosons are proportional to the square of their masses.

The choice of minimum and the assumption of a vacuum expectation value (Eqn. 1.3) are equivalent to a spontaneous breaking of the $S U(2)$ symmetry. The symmetry is no longer manifest but is said to be hidden, and the Lagrangian describes three massive gauge fields (the $W^{ \pm}$and $Z$ bosons), a massless gauge field (the photon) and one massive scalar (the Higgs boson $H)$.

\subsubsection{Yukawa Interactions}

With the replacement $\operatorname{Re}\left(\phi^{0}\right)=\frac{v+H}{\sqrt{2}}$ in Equation 1.3 that allows expansion around the vacuum, the Yukawa interactions give rise to mass terms for fermions. Taking the electron (the first lepton generation) as an example, the Lagrangian contains

$$
\mathcal{L}(e)_{Y u k a w a}=-\frac{G_{e}}{\sqrt{2}} v\left(\bar{e}_{L} e_{R}+\bar{e}_{R} e_{L}\right)-\frac{G_{e}}{\sqrt{2}}\left(\bar{e}_{L} e_{R}+\bar{e}_{R} e_{L}\right) H
$$


The electron mass is given by $m_{e}=\frac{G_{e} v}{\sqrt{2}}$. As the choice of $G_{e}$ is arbitrary, the actual value of the electron mass can not be predicted. In a similar way, all quark masses are generated. Besides the electron mass term, $\mathcal{L}(e)_{\text {Yukawa }}$ contains an interaction term coupling the Higgs scalar to the electron. The fact that the Higgs coupling to the fermions is proportional to their masses and is flavour conserving is an important experimentally testable prediction of the theory.

In summary, the choice of a single Higgs doublet is sufficient to generate the masses of the gauge bosons and fermions, but the fermion masses are not predicted and have to be taken as empirical parameters in the SM. The mass of the Higgs boson, $m_{H}$, is also not predicted.

\subsection{Limits on the Higgs Boson Mass}

The SM Higgs boson has not been observed experimentally. Various constraints are placed on possible Higgs mass values from theory, indirect measurements and direct searches.

\subsubsection{Theoretical Limits}

A very severe constraint on the Higgs boson mass comes from the requirement of unitarity of diboson scattering amplitudes [8]. From this requirement, the upper limit $m_{H} \lesssim 1 \mathrm{TeV}$ or the existence of new physics at the upper limit scale (or both) are obtained.

There are renormalisation group constraints on the Higgs boson mass. For the theory to be valid up to the Planck scale, the allowed Higgs mass window 
is $140<m_{H}<180 \mathrm{GeV}$. For a cut-off scale of new physics $\Lambda \sim 1000 \mathrm{TeV}$, the Higgs boson should lie in the mass window $110<m_{H}<300 \mathrm{GeV}[8]$.

\subsubsection{Experimental Limits}

\section{Indirect Measurements}

Fits to precision measurements of electroweak observables can indirectly provide limits on the Higgs boson mass. A global fit to measurements such as masses, cross sections and couplings of the heavy electroweak gauge bosons from the LEP, SLAC and Tevatron colliders, and elsewhere gives $m_{H}=90_{-27}^{+36} \mathrm{GeV}$ at $68 \%$ confidence level (C.L.), or $m_{H}<163 \mathrm{GeV}$ at 95\% C.L. (Fig. 1.2). If the direct limit from LEP is taken into account, the 95\% C.L. upper limit obtained is $m_{H}<191 \mathrm{GeV}[9]$.

\section{Direct Searches}

The combination of data analysed by the four LEP collider experiments (ALEPH, DELPHI, L3, and OPAL) placed a lower bound at 95\% confidence

level (C.L.) on the SM Higgs boson mass of $114.4 \mathrm{GeV}$ [10]. The principal mechanism for producing and searching for the SM Higgs boson at LEP was $e^{+} e^{-} \rightarrow H Z$.

The search continues at the Tevatron collider, using the data collected by the CDF and D $\varnothing$ collaborations. The full Tevatron combination excludes masses between $160<m_{H}<170 \mathrm{GeV}$ at 95\% C.L. (Fig. 1.3) [11] and the sensitivity is improving throughout the $115<m_{H}<185 \mathrm{GeV}$ range as the integrated luminosity increases and analysis improvements are implemented. 


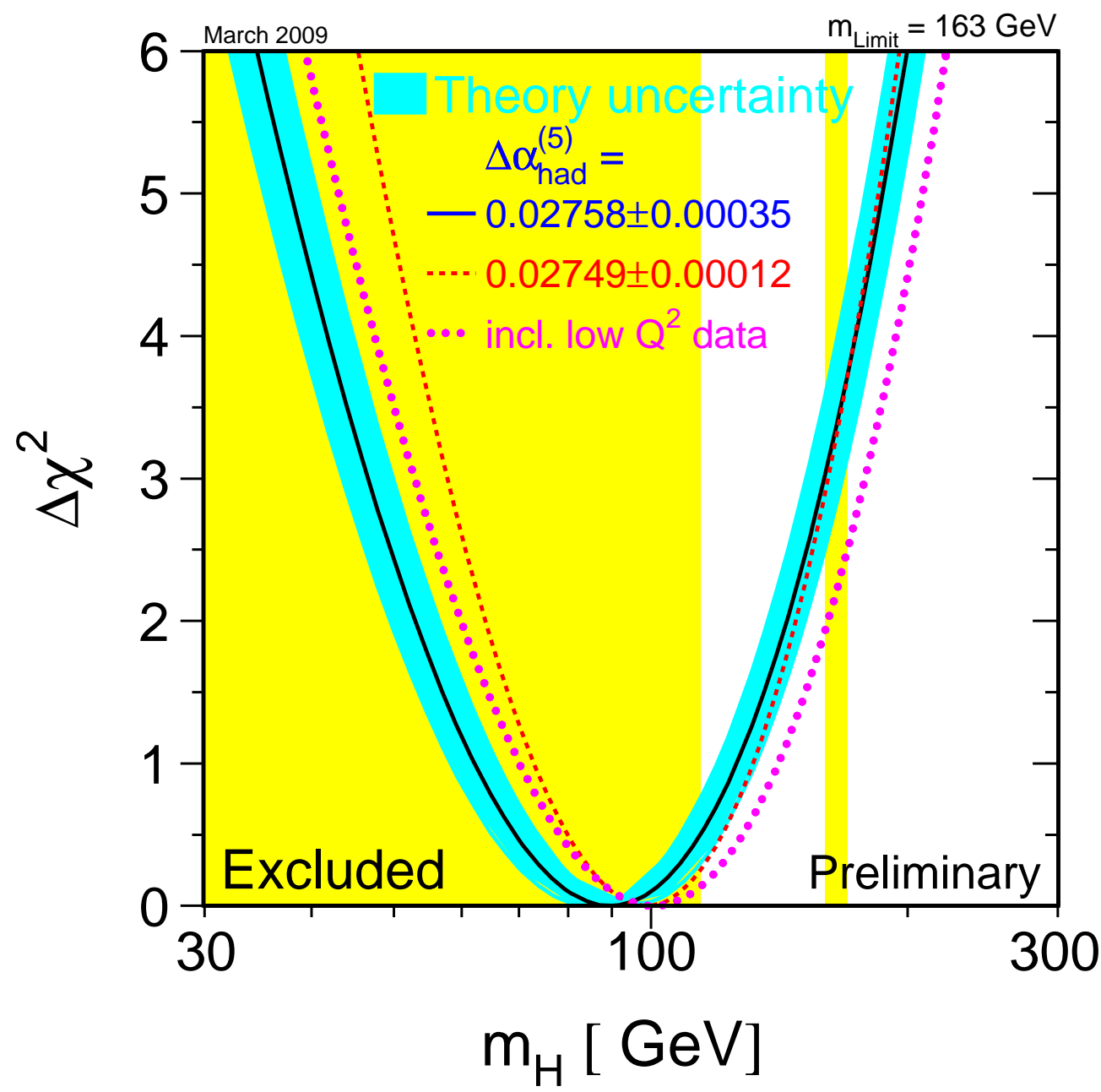

Figure 1.2: $\Delta \chi^{2} \equiv \chi^{2}-\chi_{\min }^{2}$ curve from fit to precision electroweak measurements by the LEPEWWG [9]. The preferred value for $m_{H}$, corresponding to the minimum of the curve, is $90 \mathrm{GeV}$ and the experimental uncertainty is +36 and $-27 \mathrm{GeV}$ at $68 \%$ C.L. (derived from $\Delta \chi^{2}=1$ ). The blue band corresponds to the uncertainty from theory and is not taken into account in the fit. The mass range excluded by direct searches at LEP and the Tevatron is shaded in yellow. 


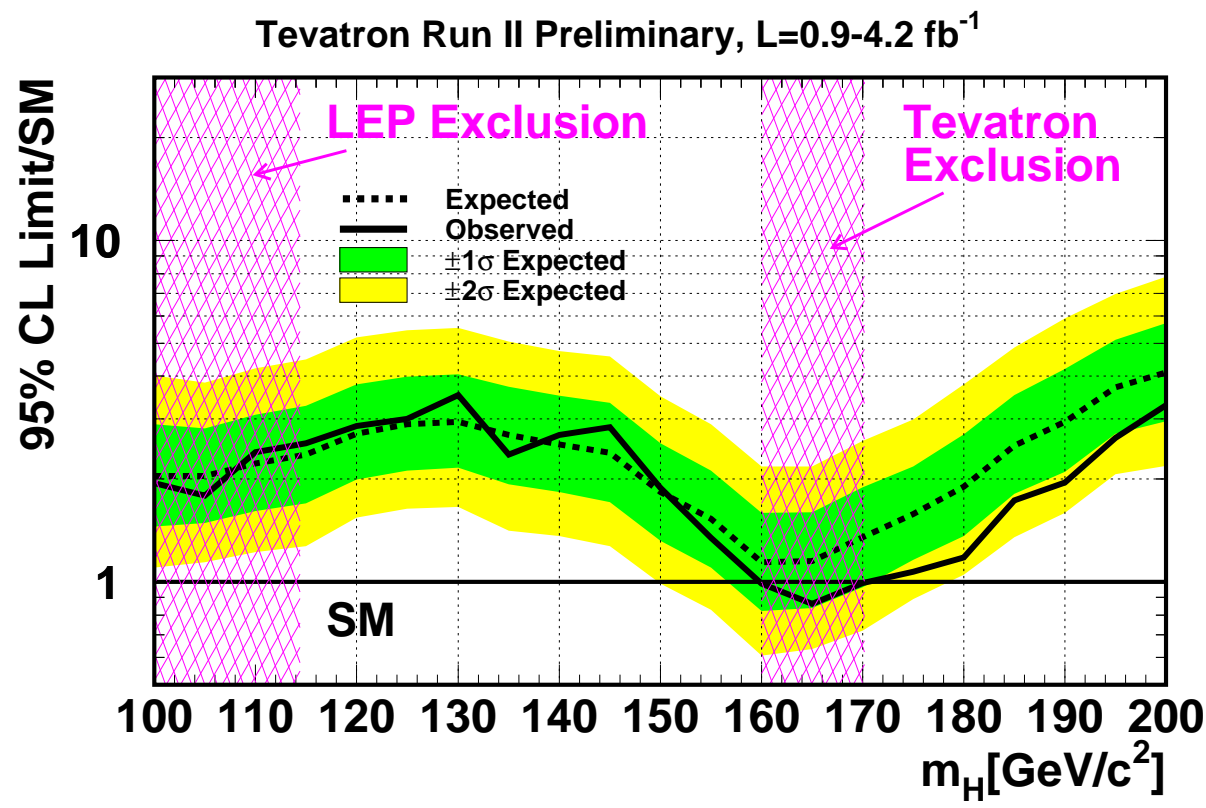

Figure 1.3: Combined CDF and DØ 95\% C.L. upper limits on Standard Model Higgs boson production as a ratio to the SM expectation. The expected limit and the 1 and 2 standard deviation bands around the median value are derived from the background prediction assuming no signal production [11].

The Large Hadron Collider (LHC) is to come into operation in the near future with significantly higher sensitivity for masses up to about $1 \mathrm{TeV}[7]$.

\subsection{Higgs Boson Production and Decay}

The dominant Higgs boson production processes in $p \bar{p}$ collisions at a centreof-mass energy of $1.96 \mathrm{TeV}$ are gluon fusion $(g g \rightarrow H)$ through a virtual $t \bar{t}$ loop and associated production with a vector boson $\left(W^{ \pm} H\right.$ and $\left.Z H\right)$ [12]. 
The SM Higgs boson decays predominantly to $b \bar{b}$ and other fermion pairs for masses up to about $135 \mathrm{GeV}$ and to $W^{+} W^{-}$at higher masses [13]. The SM Higgs boson production cross sections and decay branching ratios as a function of Higgs mass are shown in Fig. 1.4. Due to the very high background rates for multijet final states, the searches focus on associated production with a vector boson with the Higgs decaying to $b \bar{b}$ at low mass, and gluon fusion with the Higgs decaying to $W^{+} W^{-}$for intermediate and high values of the Higgs mass. An important channel in the intermediate mass range is the decay to two photons via loops, with a virtual $W^{+} W^{-}$pair providing the dominant contribution.

\section{5 $Z H \rightarrow \nu \bar{\nu} b \bar{b}$ at the Tevatron}

For Higgs searches at the Tevatron, the signal-to-background ratio is small and the signal rates are typically smaller than the systematic uncertainties on the estimated backgrounds rates. The burden of showing evidence or exclusion of the SM Higgs boson does not rest on a single channel, but on a combination of several channels. Searches in the missing energy and acoplanar $b$-jet topology have been published by CDF [14] and D $\varnothing[1,15]$.

At the Tevatron, the missing energy and acoplanar $b$-jet channel provides the best sensitivity at low mass along with the search for $W H \rightarrow \ell \nu_{\ell} b \bar{b}$, due in part to the large branching ratio of $Z \rightarrow \nu \bar{\nu}$. It is sensitive to $Z H$ associated production when the $Z$ decays to neutrinos and $W H$ production when the charged lepton from the $W$ decay is undetected (Fig. 1.5). It is complementary to searches with visible leptons in the final state. 

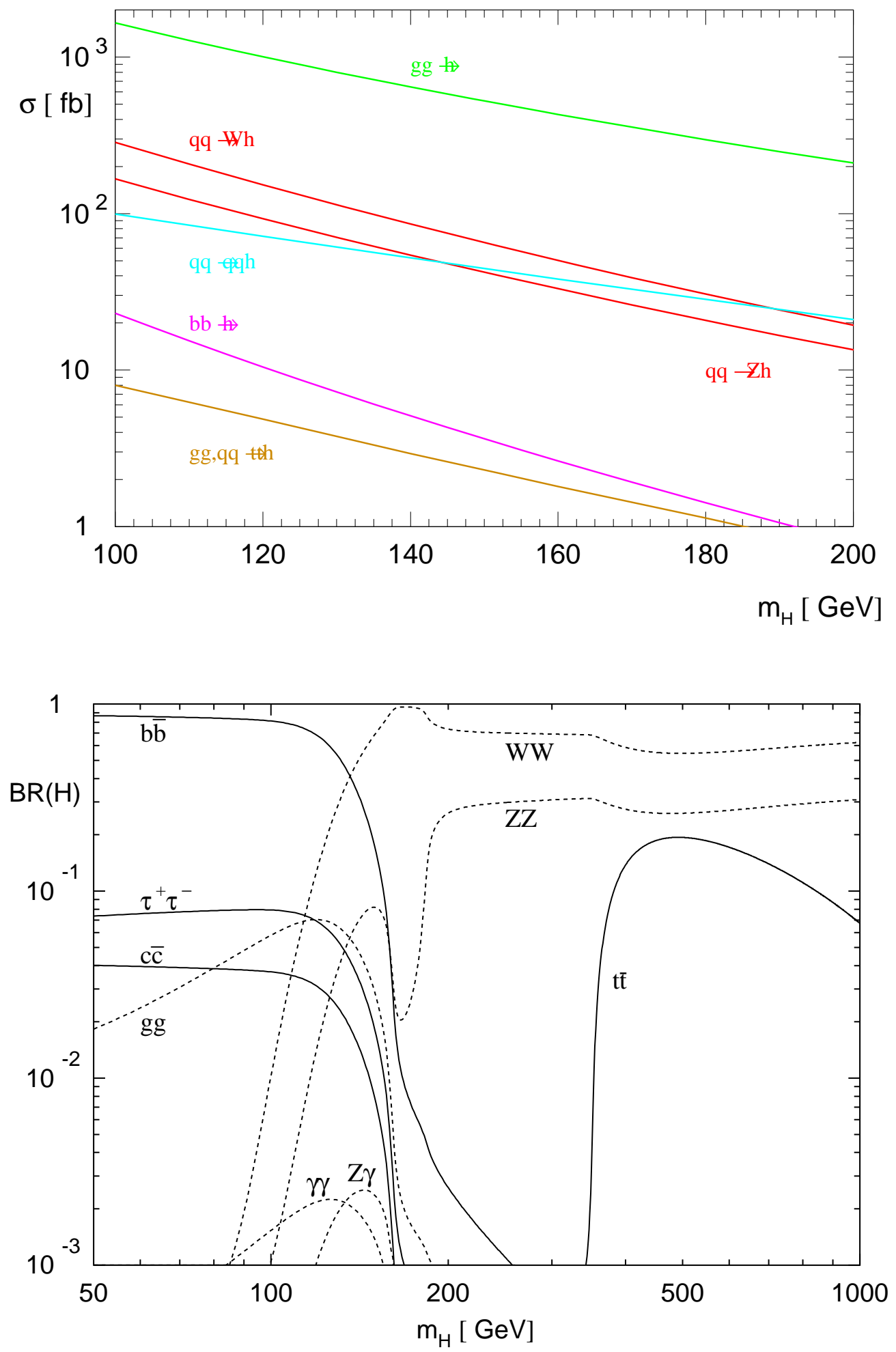

Figure 1.4: SM Higgs production cross sections in $p \bar{p}$ collisions at $1.96 \mathrm{TeV}[12]$ (top) and decay branching ratios [13] (bottom) as a function of Higgs mass. 

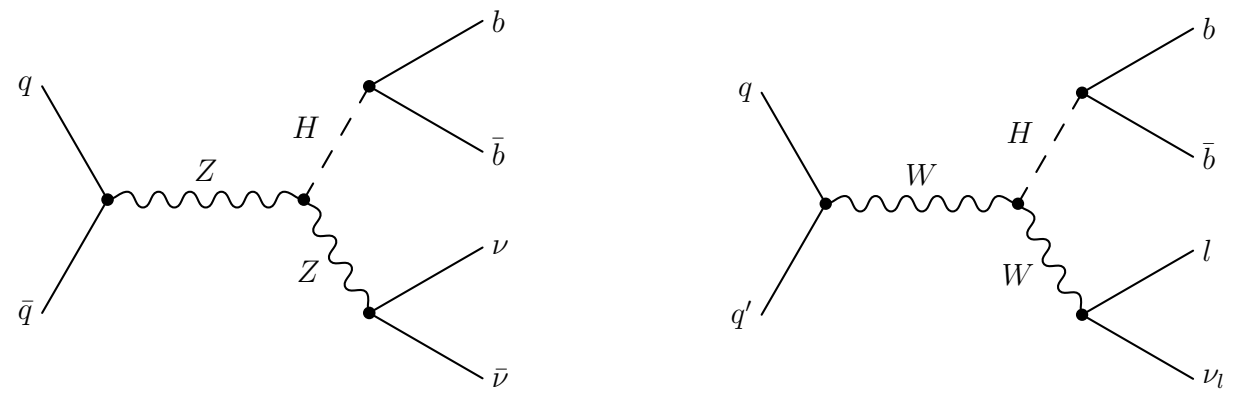

Figure 1.5: Feynman diagrams of the $p \bar{p} \rightarrow Z H \rightarrow \nu \bar{\nu} b \bar{b}$ and $p \bar{p} \rightarrow W H \rightarrow$ $\ell \nu_{\ell} b \bar{b}$ processes.

The largest sources of background are $W$ and $Z$ boson production in association with light $(u, d, s)$ and heavy $(b, c)$ quark flavour jets, top pair $(t \bar{t})$ and single top production, and diboson $(W W, Z Z$ and $W Z)$ processes. All these processes are modeled by Monte Carlo (MC) simulations. As the final state is characterised by the presence of two jets and missing transverse energy, special care is required for the rejection and modeling of multijet and instrumental backgrounds. The rates and shapes of these backgrounds are estimated using data in signal depleted regions. 


\section{Chapter 2}

\section{The Tevatron and the DØ Detector}

\subsection{The Tevatron}

The Tevatron is a superconducting synchrotron located at the Fermi National Accelerator Laboratory (Fermilab) [16]. The Fermilab accelerator complex is shown in Fig. 2.1. The Tevatron is $6.3 \mathrm{~km}$ in circumference and collides beams of protons and antiprotons at two points, where the general purpose detectors CDF and D $\varnothing$ are located. Run I of the Tevatron took place between 1992-1996 and Run II began in 2001.

\subsubsection{Proton Production}

Hydrogen ions, $\mathrm{H}^{-}$, are accelerated using a Cockcroft-Walton accelerator to $750 \mathrm{keV}$ and then by a linear accelerator (Linac) to $400 \mathrm{MeV}$. The ions pass through a fine graphite sheet which strips the electrons leaving the 
bare protons of the hydrogen nuclei. The protons are then accelerated by a synchrotron (the Booster) to $8 \mathrm{GeV}$ and passed to the Main Injector where they are accelerated to $120 \mathrm{GeV}$ for anti-proton production or $150 \mathrm{GeV}$ for injection into the Tevatron.

\subsubsection{Anti-proton Production}

Anti-protons are produced by colliding $120 \mathrm{GeV}$ protons onto a nickel target and sorting the products with a lithium lens and a magnetic field designed to act as a charge-mass spectrometer. The Debuncher and Accumulator rings are used to collect and cool the resulting anti-protons. For every $10^{7}$ protons hitting the target one anti-proton is collected at $8 \mathrm{GeV}$.

The Recycler ring which occupies the same tunnel as the Main Injector is used for further anti-proton cooling and storing. Using the Recycler as an intermediary stage gives extra freedom in choosing transfer windows from the anti-proton source to the Tevatron and helps minimise losses.

\subsubsection{Collisions}

During Run I, the Tevatron operated using six bunches each of protons and antiprotons, with 3500 ns between bunch crossings and a centre-of-mass energy of $1.8 \mathrm{TeV}$. The peak luminosity was typically $1-2 \times 10^{31} \mathrm{~cm}^{-2} \mathrm{~s}^{-1}$ and approximately $120 \mathrm{pb}^{-1}$ of data were recorded by $\mathrm{D} \varnothing$.

In Run II, which began in March 2001, the Tevatron is operated with 36 bunches of protons and antiprotons with a bunch spacing of 396 ns and at an increased centre-of-mass energy of $1.96 \mathrm{TeV}$. Run II is split into two epochs: 


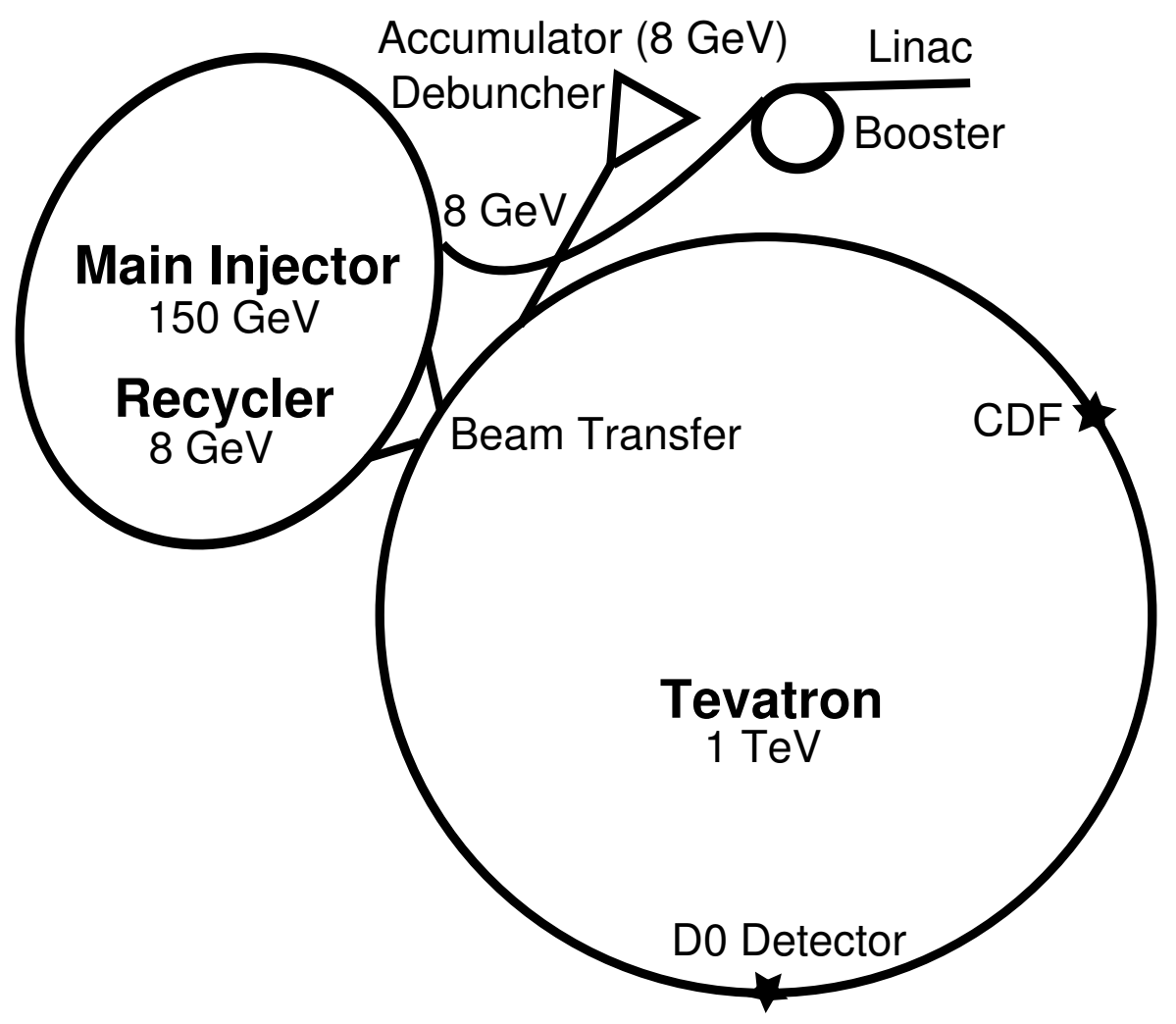

Figure 2.1: The Fermilab accelerator complex for Run II of the Tevatron.

Run IIa and Run IIb.

Run IIa finished in April 2006 with more than $1 \mathrm{fb}^{-1}$ of integrated luminosity delivered by the Tevatron. Both detectors were upgraded to operate at the higher instantaneous luminosities $\left(2-3 \times 10^{32} \mathrm{~cm}^{-2} \mathrm{~s}^{-1}[17]\right)$ in Run IIb, which began in June 2006. It is expected that up to a total of $12 \mathrm{fb}^{-1}$ of integrated luminosity will be delivered by the Tevatron to each of the detectors by the end of Run II. 


\subsection{The DØ Detector}

$\mathrm{D} \varnothing$ is a general purpose hermetic particle detector. It consists of three major subsystems: central tracking detectors, uranium/liquid-argon calorimeters, and a muon spectrometer (see Fig. 2.2). All sub-detectors are described in more detail in the following sections, and full details can be found in $[18,19]$.

In the detector description and data analysis, DØ's customary righthanded coordinate system is used, in which the z-axis is along the proton beam direction and the y-axis is upward. The angles $\phi$ and $\theta$ are the azimuthal and polar angles respectively. The $r$ coordinate denotes the perpendicular distance from the z-axis. The pseudorapidity, $\eta=-\ln [\tan (\theta / 2)]$, approximates the true rapidity, $y=(1 / 2) \ln \left[\left(E+p_{z}\right) /\left(E-p_{z}\right)\right]$, for finite angles in the limit that $(m / E) \rightarrow 0$. The term "forward" is used to describe the regions at large $|\eta|$. Distance in $\eta-\phi$ space is defined as $\Delta \mathcal{R}=\sqrt{(\Delta \eta)^{2}+(\Delta \phi)^{2}}$.

\subsubsection{Central Tracking System}

The DØ central tracking system comprises the Silicon Microstrip Tracker (SMT) and the Central Fibre Tracker (CFT) inside a solenoidal magnet (see Fig. 2.3). The generated magnetic field allows for charge sign determination and momentum calculation for charged particles.

\section{The Silicon Microstrip Tracker}

The SMT, shown in Fig. 2.4, provides tracking and vertexing for almost the full $\eta$ range of the calorimeter and muon detectors, using 792576 read out 


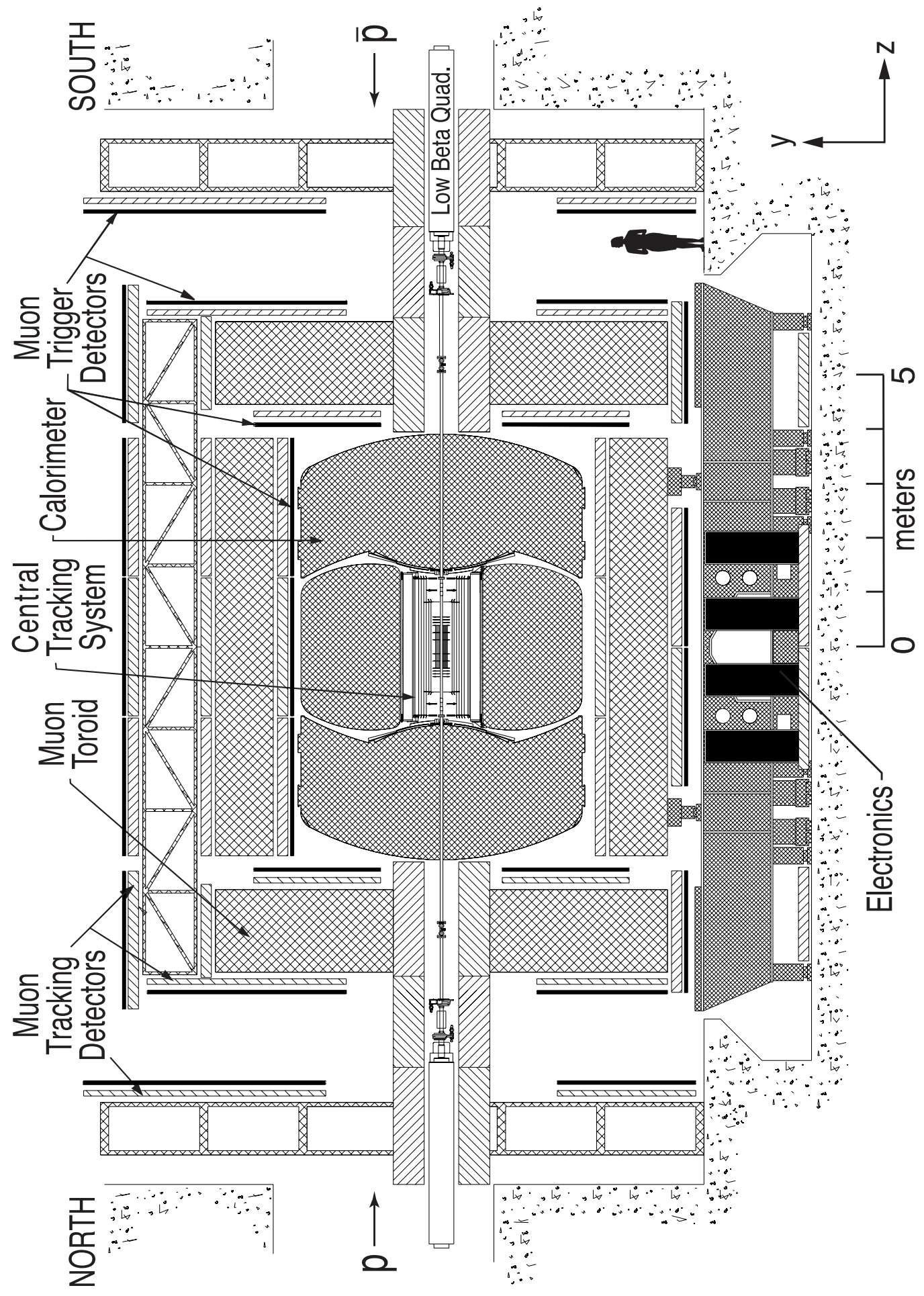

Figure 2.2: Cross-sectional view of the D $\varnothing$ detector [18]. 


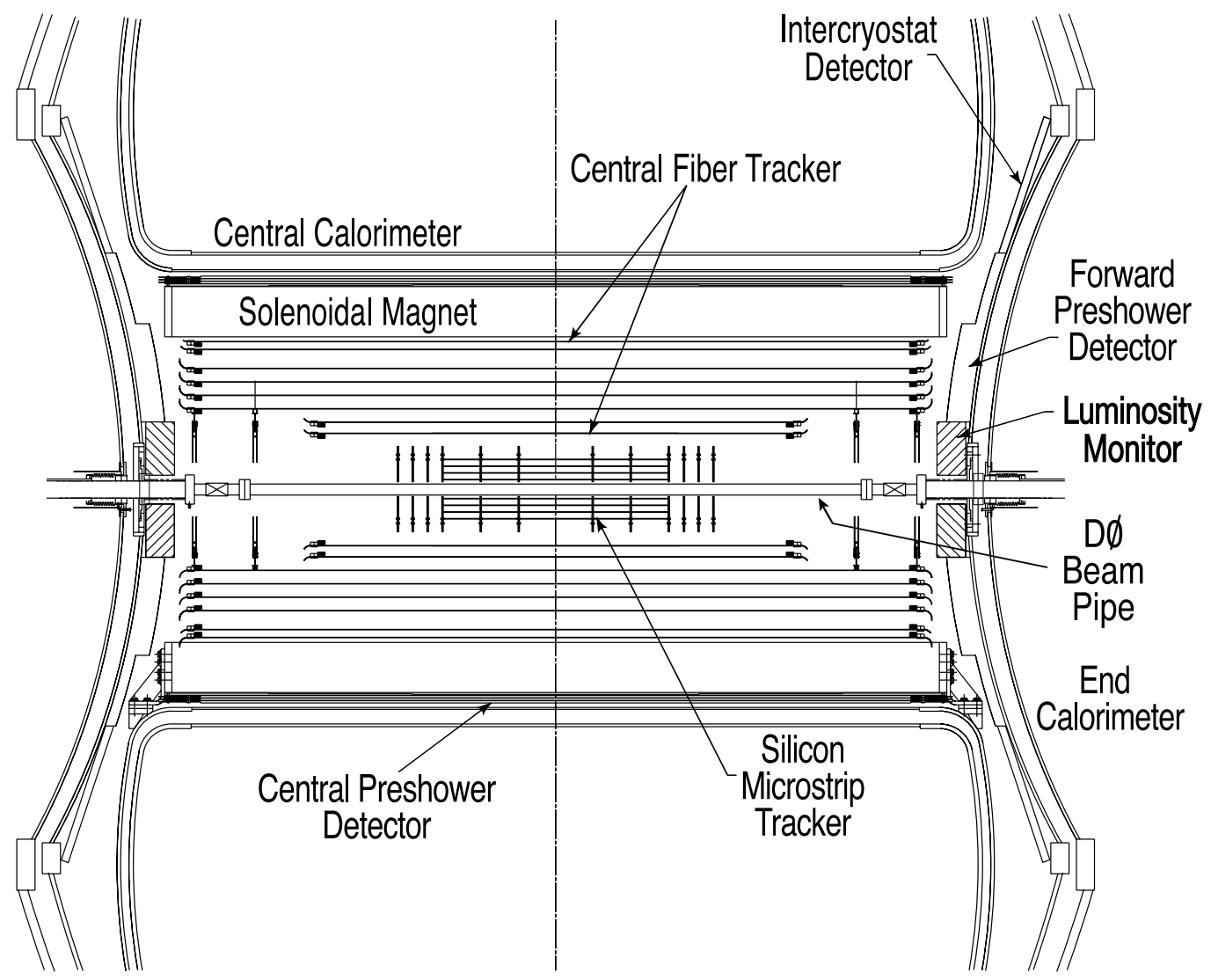

Figure 2.3: Cross-sectional view of the central tracking system in the $x-z$ plane. Also shown are the locations of the solenoid, the preshower detectors, luminosity monitor, and the calorimeters [18]. 


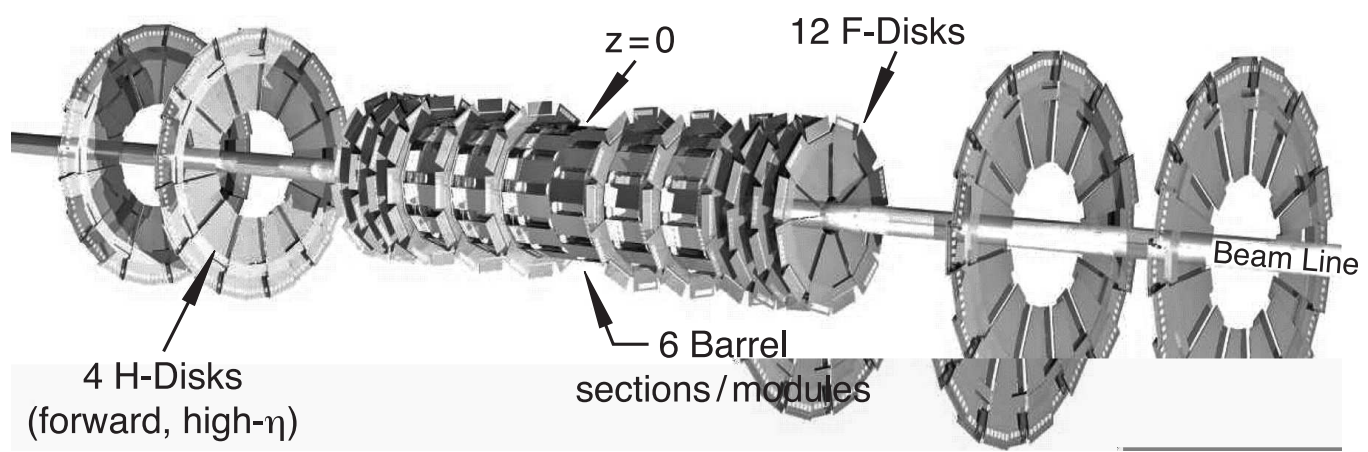

Figure 2.4: The disk/barrel design of the silicon microstrip tracker [18].

channels. Around $90 \%$ of the sensors are functional. The SMT detector is made from high precision silicon wafers arranged into three subgroups:

Barrels There are 6 barrel detectors in total, each made from 4 concentric layers of double sided rectangular silicon wafers. The barrel detectors cover $2.7 \mathrm{~cm}<r<10.5 \mathrm{~cm}$ and $|\mathrm{z}|<38 \mathrm{~cm}$, providing tracking in the region $|\eta|<2.4$.

F-Disks There are 12 F-disks, 6 cap each barrel at high $\mathrm{z}$ and 2 triplets of F-disks spaced 5, 10 and $15 \mathrm{~cm}$ from either end of the barrel detectors. Each F-disk is constructed from 12 double sided wedge shaped silicon modules.

H-Disks The H-disks are designed for high $\eta$ coverage. Two doublets are placed at $1 \mathrm{~m}$ on either side of the barrel detector. Each H-disk is made from 24 wedges, each wedge is constructed from back to back single sided silicon modules. The H-disks extend the coverage of the SMT tracking in the forward region up to $|\eta|<3$. 
As part of the Run IIb upgrade an additional layer of silicon has been added to the detector, referred to as Layer-0. Layer-0 is located inside the Run IIa barrel detector at a radius of $1.6 \mathrm{~cm}$, and its purpose is to recover performance loss due to radiation damage and improve the $b$-tagging resolution.

\section{The Central Fibre Tracker}

The CFT is made of scintillating fibres of diameter $835 \mu \mathrm{m}$ mounted onto eight concentric cylinders. The fibres form stereo and axial doublets to allow hit separation in the $\mathrm{z}-\theta$ plane. There are ribbons of 256 fibres in each doublet layer.

The scintillating fibres are made up of a polystyrene core surrounded by an acrylic layer and an outermost flouro-acrylic layer. They are connected to clear waveguides which carry the light to visible light photon counters (VLPC) where the light is converted to an electrical signal. The VLPCs

have a fast response time, a quantum efficiency of greater than $75 \%$ and a high gain of $2.2-6.5 \times 10^{4}$. The CFT requires 76,800 channels of VLPC read out.

Working together, the SMT and CFT detectors locate the primary interaction vertex with a resolution of about $35 \mu \mathrm{m}$ along the beamline. They can tag $b$-quark jets with an impact parameter resolution of better than $15 \mu \mathrm{m}$ in $r-\phi$ for particles with transverse momentum $p_{T}>10 \mathrm{GeV} / c$ at $|\eta|=0$. The transverse momentum $\left(\mathrm{p}_{T}\right)$ resolution is of the order of

$$
\frac{\delta \mathrm{p}_{T}}{\mathrm{p}_{T}}(\text { in } \%)=2+0.2 \times \mathrm{p}_{T}(\text { in } \mathrm{GeV}) .
$$




\section{The Solenoidal Magnet}

The 2 T superconducting solenoid was designed to optimise the momentum resolution. The size of the solenoid was determined by the size of the calorimeter and the tracking requirements and it is $2.73 \mathrm{~m}$ in length, $1.42 \mathrm{~m}$ in diameter, and 1.1 radiation lengths, $X_{0}$, thick. The magnetic field is uniform to $0.5 \%$ within the tracking volume.

\subsubsection{Calorimeter}

Calorimetry is the process of identifying photons, electrons and jets and measuring their energy. Calorimetry at D $\varnothing$ is conducted by preshower detectors, by the Central and End Calorimeters and by an intercryostat detector.

\section{The Preshower Detectors}

Triangular scintillating tiles called preshower (PS) detectors are used for calorimetry and tracking, facilitate electron identification, background rejection and aide in matching central tracks to calorimeter clusters. The central preshower (CPS) detector is placed between the solenoid and the central calorimeter and covers $|\eta|<1.3$ and the Forward Preshower detectors are attached to the inner faces of the end calorimeters and cover $1.5<|\eta|<2.5$ (See Fig. 2.3).

\section{The Calorimeter}

DØ uses a sampling calorimeter divided into a central and two end components (see Fig. 2.5). Each calorimeter component is enclosed in a cryostat 


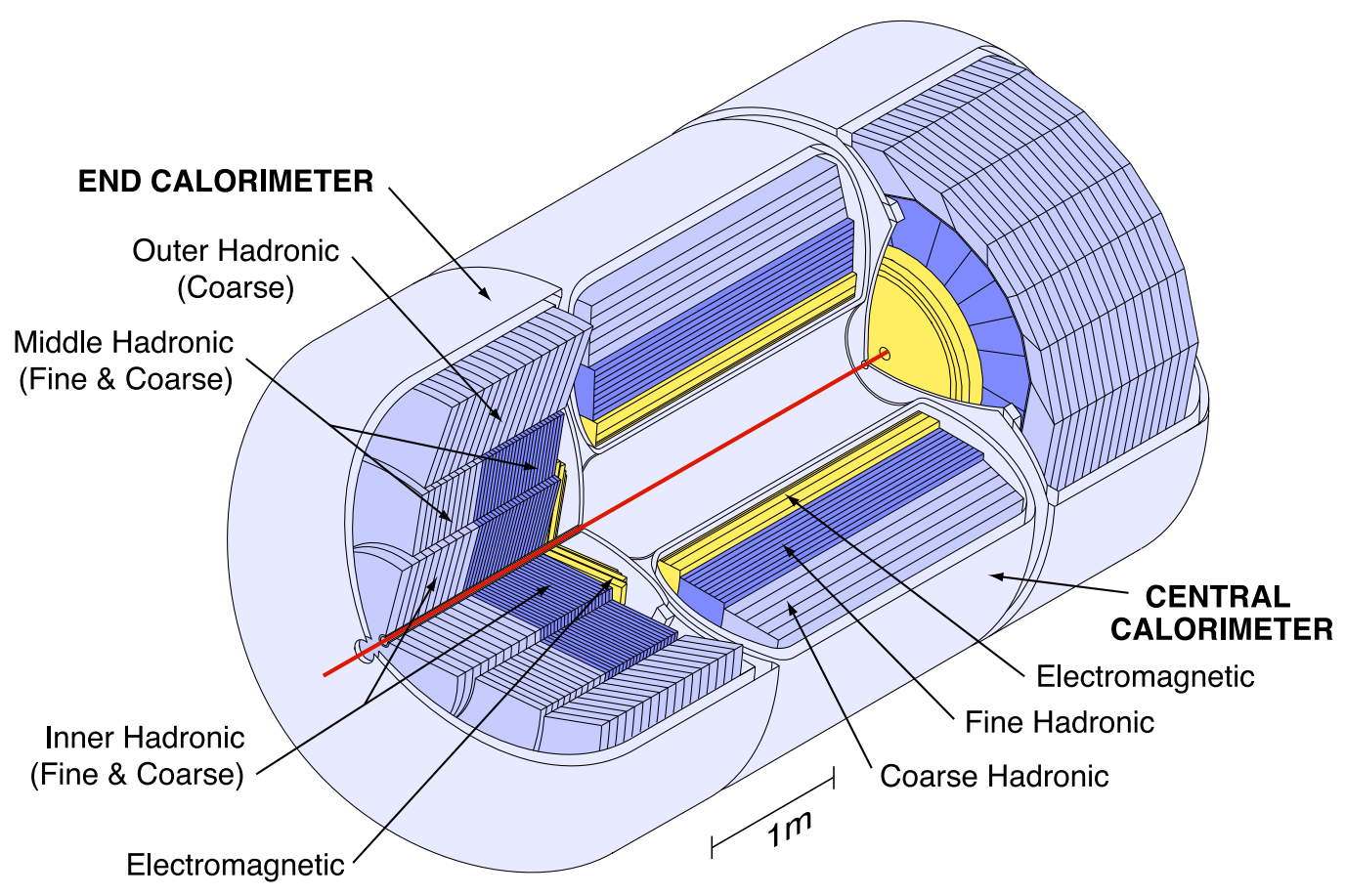

Figure 2.5: The DØ Run II calorimeter [18].

to keep the sampling medium (liquid Ar) at approximately $90 \mathrm{~K}$. There are 55296 calorimeter read out channels.

The central calorimeter $(\mathrm{CC})$ section provides coverage up to $|\eta| \sim 1$ and comprises three concentric regions along $r$ : the electromagnetic (3 layers, 20 radiation lengths $X_{0}$ ), the fine hadronic (3 layers, 3.1 nuclear absorption lengths $\left.\lambda_{A}\right)$ and the coarse hadronic $\left(3.2 \lambda_{A}\right)$.

The two end calorimeters (EC) provide complementary coverage up to $|\eta| \sim 4$ and have four regions each: the electromagnetic (4 layers, 21.4 $X_{0}$ ), the inner hadronic, the middle hadronic and the outer hadronic. The hadronic regions are further divided up into fine (inner: $4.4 \lambda_{A}$, middle: 3.6 $\lambda_{A}$ ) and coarse (inner: $4.1 \lambda_{A}$, middle: $4.6 \lambda_{A}$, outer: $6.0 \lambda_{A}$ ) sections.

Different sections use different absorbers: the electromagnetic (EM) layers 
use 3-4 mm thick uranium, the fine hadronic ( $\mathrm{FH}$ ) layers use $6 \mathrm{~mm}$ thick uranium and the coarse hadronic $(\mathrm{CH})$ layers use $46.5 \mathrm{~mm}$ thick plates of copper (CC) or stainless steel (EC). The EM layers are designed to collect most of the EM energy, the FH layers most of the hadronic energy and the $\mathrm{CH}$ layers any leakage. Towers in both EM and hadronic modules have a width of $\Delta \eta=0.1$ and $\Delta \phi=2 \pi / 64 \sim 0.1$.

The energy resolution provided by the calorimeter has been measured using test beam data and found to be:

$$
\begin{aligned}
& \frac{\sigma_{E}}{E}=15.7 \% / \sqrt{E}+0.3 \%(\mathrm{EM}) \\
& \frac{\sigma_{E}}{E}=41.0 \% / \sqrt{E}+3.2 \%\left(\pi^{ \pm}\right)
\end{aligned}
$$

where the energy $E$ is measured in $\mathrm{GeV}$.

\section{The Intercryostat Detector (ICD)}

The intercryostat region (ICR) has incomplete coverage by the calorimeter. A detector covers the region $1.1<\eta<1.4$ and provides additional sampling. It consists of scintillating tiles mounted on the cryostat walls of the EC calorimeters. There are 12 tiles in total and each is read out in twelve subdivisions of size $\Delta \eta \times \Delta \phi \sim 0.1 \times 0.1$.

\subsubsection{The Muon Spectrometer}

The muon spectrometer is the outermost layer of the detector (Fig. 2.2). It is split into two systems: the central muon system which provides coverage up 
to $|\eta|<1$ and the forward muon system which covers the region $1<|\eta|<2$.

Each system has three layers of drift tubes; Layer A lies inside a $1.8 \mathrm{~T}$ solid iron toroid magnet and B/C lie outside. The central muon system uses proportional drift tubes (PDT) and the forward system mini drift tubes (MDT). Layer A has four decks of drift tubes and layers B/C have three.

The central muon system has two layers of scintillator counters, one before Layer A and one after Layer C. The forward muon system has three layers of scintillator counters. The scintillator counters facilitate quick trigger decisions on muons, and provide accurate timing information for track reconstruction in the drift chambers. They also allow reduction of backgrounds such as cosmic rays.

The scintillator counters have a time resolution of $\sim 2 \mathrm{~ns}$, and both the PDT and MDT have a hit resolution of $\sim 1 \mathrm{~mm}$. The standalone momentum resolution of the forward muon system is approximately $20 \%$ for muon momentum below $40 \mathrm{GeV} / \mathrm{c}$. The muon system improves the momentum resolution achieved by the tracking detectors for muons with momentum greater than $100 \mathrm{GeV} / \mathrm{c}$ and for those that do not go through all the CFT layers.

\subsubsection{Luminosity Monitor}

The luminosity monitor $(\mathrm{LM})$ is used to determine the luminosity $(\mathcal{L})$ at the interaction point by detecting inelastic $p \bar{p}$ collisions. Two arrays of 24 plastic scintillation counters are placed at $\mathrm{z}= \pm 140 \mathrm{~cm}$, just in front of the EC calorimeter and in the region between the beampipe and the frontal preshower detectors (see Fig. 2.3). 
The luminosity $\mathcal{L}$ is determined from the average number of inelastic collisions per beam crossing $\bar{N}_{L M}$ :

$$
\mathcal{L}=\frac{f \bar{N}_{L M}}{\sigma_{L M}}
$$

where $f$ is the beam crossing frequency and $\sigma_{L M}$ is the effective cross section for the LM [20]. $\bar{N}_{L M}$ is determined from the fraction of beam crossings with no collisions using Poisson statistics.

\subsubsection{The Trigger}

A powerful and flexible trigger is the cornerstone of a modern hadron collider experiment. The trigger system at D $\varnothing$ consists of three levels, as shown in Figure 2.6. Different levels are used to allow for both a high degree of rejection and also for a high degree of flexibility when making triggering decisions. The trigger at $\mathrm{D} \varnothing$ has three levels of increasing complexity referred to as Level-1 (L1), Level-2 (L2) and Level-3 (L3). Each level has increasingly sophisticated event reconstruction, and an event will proceed through each of the trigger levels dependent on conditions being met. The trigger rates at each level of the trigger and the latency are shown in Table 2.1. A program called COOR handles the overall coordination and control of the trigger system, and interacts directly with the trigger framework (which makes the L1 and L2 trigger decisions). 


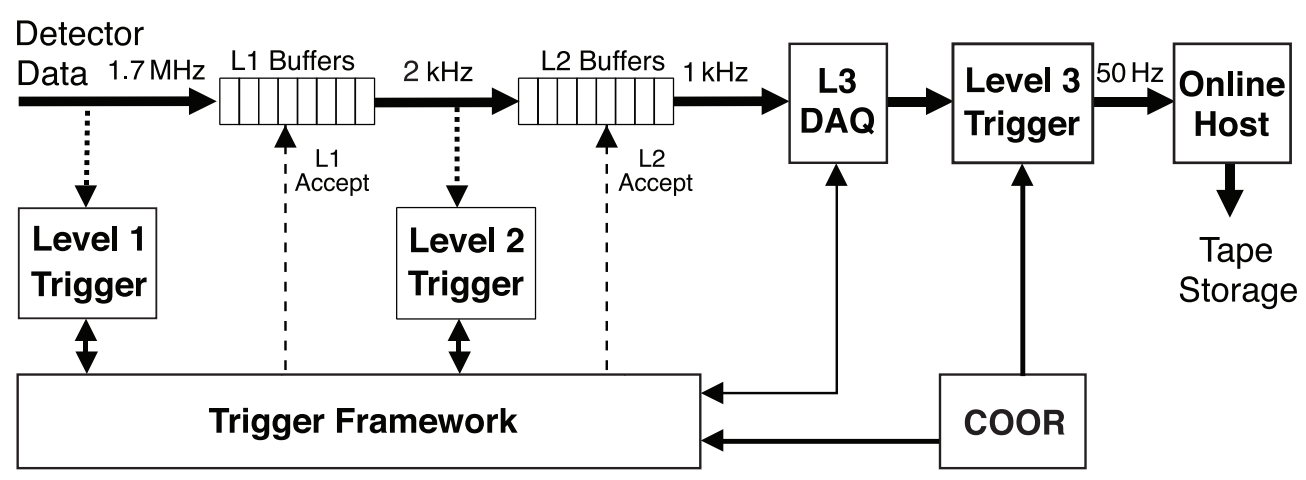

Figure 2.6: Overview of the $\mathrm{D} \varnothing$ trigger and data acquisition systems [18].

\begin{tabular}{ccc} 
& Rate & Latency \\
\hline Collisions & $1.7 \mathrm{MHz}$ & \\
Level-1 & $1.6 \mathrm{kHz}$ & $3.6 \mu \mathrm{s}$ \\
Level-2 & $800 \mathrm{~Hz}$ & $\sim 100 \mu \mathrm{s}$ \\
Level-3 & $50 \mathrm{~Hz}$ & $\sim 150 \mathrm{~ms}$ \\
\hline
\end{tabular}

Table 2.1: Approximate trigger rates and latency for the three trigger levels.

\section{Level-1}

The Level-1 trigger involves a series of Field Programmable Gate Arrays (FPGAs), installed on certain of the sub-detectors. These include a reduced form of the readout electronics for the calorimeter, the axial components of the CFT and preshower detectors and also the muon systems. The results from each of these components are logically combined into up to 128 different triggers. If all the requirements for a particular trigger are satisfied, a Level-1 trigger bit is created and the data are digitised and buffered for processing in the next level trigger, Level-2. The maximum decision time for Level-1 is $4.2 \mu \mathrm{s}$ and it has no dead-time.

For the calorimetry a decision is required if the energy deposited within a region, known as a trigger tower $(\Delta \phi \times \Delta \eta=0.2 \times 0.2)$, is above a threshold. 
The result is then passed to the combination process to decide if the data should be kept. The CFT trigger uses only the axial component of the CFT and tests whether hit patterns that have been created within $4.5^{\circ}$ sectors of the CFT are consistent with programmable $p_{T}$ thresholds. This can then be matched to energy deposits in the central preshower (CPS). The other main component of the Level-1 trigger system is the muon system. It uses information from the muon scintillation counters and wire chambers and matches them with data from the Level-1 tracking component.

For Run IIb a newly designed L1 track trigger is utilised, which generates track-based triggers and provides tracking information to several other trigger systems. A new L1 calorimeter trigger also replaced the old one. The calorimeter upgrade employs digital filtering to associate energy with the correct beam crossing in the Run IIb environment and provides the capability of clustering energy from multiple trigger towers.

\section{Level-2}

The Level-2 trigger consists of a combination of FPGAs and microprocessors to perform a more detailed analysis of the data passed on by Level-1. It is designed to handle a $1.6 \mathrm{kHz}$ input from Level-1 and to provide a factor of 10 rejection, resulting in an output rate of $200-900 \mathrm{~Hz}$. The maximum decision time for L2 is $100 \mu s$ with a maximum dead-time of 5\%. Level-2 uses all the data from each of the detector components. For each Level-1 trigger bit there is a corresponding Level-2 trigger bit. The information from the data passed on from Level-1 is combined and used to produce a decision.

The individual components of the detector: calorimetry, tracking and 
muon systems, each have a dedicated preprocessor system which handles the data analysis for that component and also finds and marks the possible presence of physics objects (for example jets or electrons). The results from the preprocessors are then passed onto the L2 global processor which, like the L1 system, combines the data to produce a L2 trigger bit if the given conditions are passed. The triggering conditions depend upon the corresponding L1 trigger bit and can involve a combination of data from different detector components. If a L2 trigger bit is set, the information is passed to the Level-3 trigger.

\section{Level-3}

The Level-3 trigger is entirely software based, and consists of a high performance computing farm that performs a partial event reconstruction. Each node of the farm runs an independent instance of the L3 code on complete events sequentially. If the criteria are satisfied, the data are sent to the data logger to be written to tape.

Almost all of the code for the reconstruction and programmable triggers (Levels 2 and 3 ) is written using the $\mathrm{C}^{++}$programming language. The algorithms that process the event data are known as tools. Tools are called by filters and tools can also call other tools. There are several different types of physics, unpacking and trigger tools. High level physics tools are described in Sec. 3.3. The parameters used by the tools can be modified through the use of L3 filter scripts. A filter script, which can comprise several filters, is associated with each L3 trigger bit. Filters can be either physics object filters, which apply selection cuts on the reconstructed physics objects, or 
relational filters, which compare the results to those of other filters.

An event is passed by the trigger if all the filters for any one of the filter scripts pass. It would be possible for processing to stop at this point. However, there is little performance gain in stopping processing as soon as an event passes the trigger and it simplifies timing analysis and luminosity calculations if it is not stopped. For this reason all the relevant filter scripts are always run on an event, even after it is accepted.

\subsubsection{Offline Reconstruction}

After an event has been passed by the trigger system and written out to tape, it will undergo a full event reconstruction on an offline CPU farm. The reconstruction time depends on the instantaneous luminosity and typically is $\sim 20 \mathrm{~s}$. The various versions of $\mathrm{D} \varnothing$ code which are used to reconstruct the offline physics objects are referred to by the terminology pXX, where XX is an integer incremented for each new major release of the code. 


\section{Chapter 3}

\section{Level-3 Trigger Impact}

\section{Parameter $b$-tagging}

\subsection{Introduction}

Many physics studies at D $\varnothing$ - from top quark measurements to Higgs boson searches - require the identification of $b$ quarks ( $b$-tagging). It is thus desirable to have algorithms for selecting events with high $b$-quark content probability when trigger decisions are made. Because of the limited rate-totape, the rarity of the interesting processes and the very high background rates at hadron colliders, it is important to achieve high efficiency, purity and background rejection. Online processing time constraints place limitations on the complexity of the algorithms implemented in the trigger.

$B$-hadrons have a long lifetime, large mass, high decay multiplicity and substantial leptonic branching rate compared to light quark jets. In particular, the $B$-hadron lifetimes are about 1.6 ps [21], corresponding to a decay 
flight of about $3 \mathrm{~mm}$ for a momentum of $40 \mathrm{GeV} / \mathrm{c}$. The Level-3 impact parameter $b$-tag tool uses the information from several other Level-3 tools to calculate probabilities for a jet or event to originate from the primary vertex. The Level-3 b-tagging algorithm uses a probability distribution, parametrised from lifetime information in data, to produce an overall probability that the event originated from heavy or light quark production.

$b$-tagging was introduced in the highest level trigger and certified during Run IIa [22], but changes in the tracking algorithm, the inclusion of Layer 0 in the SMT tracker and the high instantaneous luminosities necessitated the re-parametrisation and re-certification of the algorithm for Run IIb. The underlying track based probability distribution was re-parametrised using data taken at higher luminosities and certified over a sample of events that were $b$-tagged using the D $\varnothing$ offline Neural Net (NN) b-tagging algorithm [23].

\subsection{Signed Impact Parameter}

Lifetime tagging can be used to identify events with $b$-quark content. Reconstructed tracks originating from the decays of b-quarks, when extrapolated backwards, do not pass exactly through the primary interaction vertex. The Impact Parameter (IP) is defined as the minimal distance between the estimated primary interaction point and the track trajectory. Therefore, the decay of a long-lived particle produces tracks with large impact parameters, which is not the case for particles from the primary interaction.

The sign of the IP is set to be negative (positive) if the point of closest approach of each track to the estimated particle flight path is upstream 
(downstream) of the primary interaction vertex (PV) position. The IP significance is the ratio of the measured value of the IP over the expected precision of the measurement. The expected precision is a convolution of the uncertainties in the track-vertex distance of closest approach (DCA) measurement and in the spatial coordinates of the PV. The significance is assigned the same sign as the IP.

Tracks from decays of long-lived particles have predominantly positive IP signs while tracks coming directly from the PV are equally likely to have a positive or negative IP. The distribution of the negative track significance is determined mainly by tracks coming from the PV, including scatters in the detector material and tracks with wrong hit association. The excess of positively signed tracks with large significance is observed in the plot of Fig. 3.1. For $b$-tagging, only tracks with positive IP are used, thus reducing by half the number of background tracks.

\subsection{The Level-3 Physics Tools}

The $b$-tagging tools are high level trigger tools, processing physics objects returned by other tools rather than handling raw data. The following section provides an overview of the lower level tools and the relevant physics objects that they generate and provide as input to the higher level tools. Fig. 3.2 is a schematic representation of the dependencies and information flow across the different tools. 


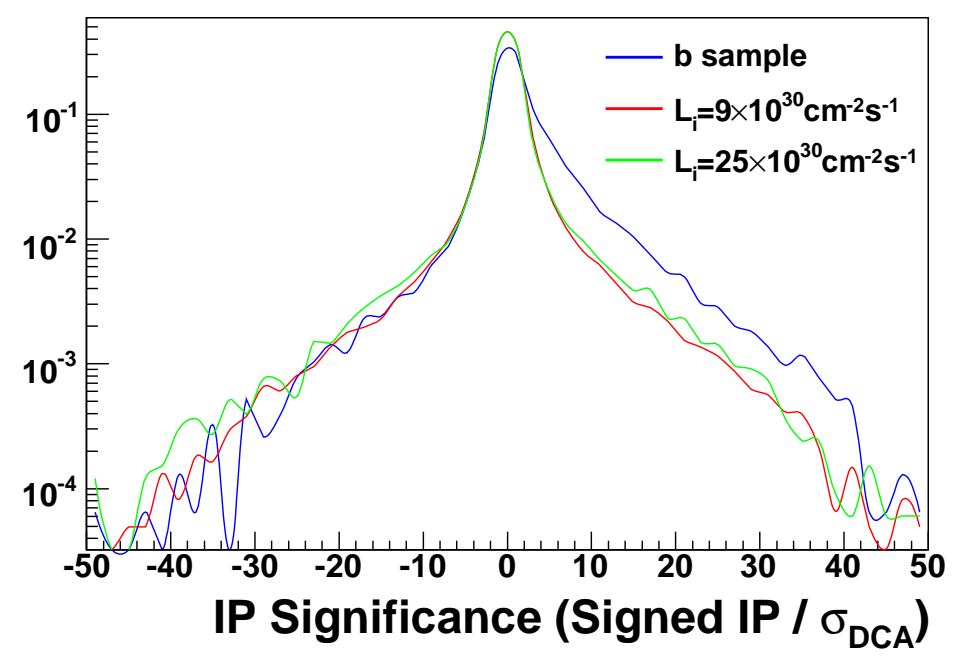

Figure 3.1: Normalised signed impact parameter distributions for events from runs with different initial luminosities and for a sample of events with enhanced $b$-content. Details about the sample selection can be found in section 3.7 .

\subsubsection{The Level-3 Tracker}

The raw output of detector components in both the SMT and CFT is passed through an analogue to digital converter (ADC). Pedestal counts are subtracted from the ADC count in each readout channel to remove noise. If the number of counts is greater than a predefined threshold after the pedestal subtraction, it is considered a hit. SMT and CFT unpacking tools [24] are then used to construct 'clusters' $[25,26]$. The position of each cluster is given by the mean of the magnitude-weighted hit positions in adjacent hit components of each sub-detector. The total ADC count of all hits in a cluster must exceed a threshold. Level-3 clusters are constructed in the $x-y$ (axial) and $z$ (stereo) detector layers. 


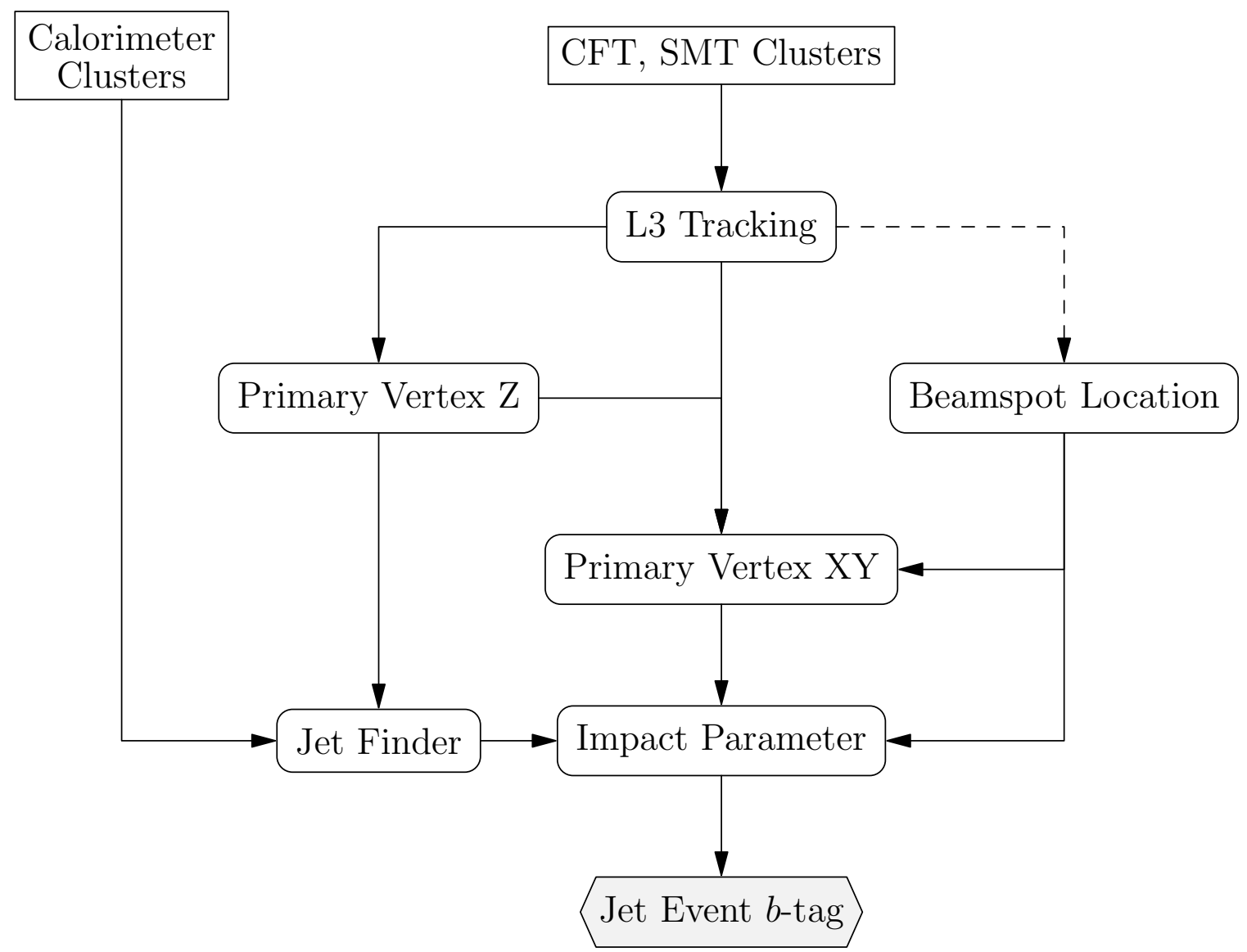

Figure 3.2: Flow chart of information in Level-3 tools for $b$-tagging. The dashed line denotes flow of non-realtime information from the previous data taking run. 
The tracking algorithm [27] uses the clusters in the CFT and SMT barrel layers (including layer 0) to reconstruct track helices. A minimum of $7 \mathrm{CFT}$ axial hits is required for each track. Multiple scattering and non-solenoidal magnetic field effects are neglected. In the CFT, both axial and stereo candidate roads are determined using a histogramming technique. Given a candidate road, nearby clusters are sought to build the track candidates. The SMT tracking uses a projective approach starting from a CFT track.

The L3 tracker achieves track finding efficiencies of 70-30\% with respect to the offline tracking algorithm with a corresponding purity (tracks matched to offline) of $48-30 \%$ for luminosities ranging from $1-3 \times 10^{32} \mathrm{~cm}^{-2} \mathrm{~s}^{-1}$ [27].

\subsubsection{The Level-3 Vertex Finder}

The Level-3 vertex finder [28] takes tracks as input. The $z$-position of the vertex is found using a $p_{T}$ weighted histogramming technique. The tracks inside the two adjacent $1 \mathrm{~cm}$ bins with the highest summed $p_{T}$ on the $z$-axis are selected. The $z$-position is taken to be the mean DCA from the origin $\left(Z_{0}\right)$ of the selected tracks. Using the track $p_{T}$ information makes the method more robust against secondary vertices.

To find the vertex position in the $x-y$ plane, an impact parameter minimisation routine is used. The tracks are approximated by straight lines at the minimum distance from the beamspot position and a $\chi^{2}$ quantity that takes into account the impact parameter and its error for each track is minimised. The nominal beamspot is taken to be the average position of primary vertices from the previous data taking run and is parametrised as a 
function of $z$.

The performance of the vertex finder tool was evaluated on different MC processes. Both the efficiency and purity are very high for a multitude of topologies $(\sim 97 \%)$. For data from a physics run, the efficiency of finding a $z$-vertex is $\sim 94 \%$ and given a $z$-vertex, the $x-y$ vertex finding efficiency is $\sim 81 \%[28]$.

\subsubsection{The Level-3 Jet Finding Algorithm}

The L3 jet finding algorithm [29] is a simplified version of the jet reconstruction algorithm run offline. One of the main differences is that there is no jet energy scale correction applied online. Jets are formed using clusters of calorimeter hits within cones of $\Delta R<0.5$. For $b$-tagging purposes, a track is attached to the nearest jet online if the angle between them is less than $45^{\circ}$.

\subsection{The Level-3 IP $b$-tagging tool}

The Level-3 impact parameter $b$-tag tool [30] uses the information from several other Level-3 tools to calculate probabilities for a jet or event to be consistent with originating from the primary vertex . Inside the tool, tracks from the Level-3 tracker are selected to pass the following criteria:

- The minimum $p_{T}$ of a reconstructed track is required to be $0.4 \mathrm{GeV}$. This value has a great impact on the L3 CPU processing time.

- General track quality requirements based on quantities from the tracker:

- Track fit $\chi^{2}$ over the number of degrees of freedom, $\chi^{2} /$ n.d.f. $<5$ 
- Number of SMT hits, $N_{S M T} \geq 1$,

- 2D distance of closest approach between track and primary vertex, $\mathrm{DCA}_{r \phi}<0.5 \mathrm{~cm}$

- Uncertainty on the 2D DCA, $\sigma_{\mathrm{DCA}}<0.075 \mathrm{~cm}$.

- Jet matching:

$-\hat{p}_{\text {jet }} \cdot \hat{p}_{\text {track }}>0.7$, where $\hat{p}_{\text {jet }}$ and $\hat{p}_{\text {track }}$ are the $3 \mathrm{D}$ jet and track momentum directions,

- jet rank $\leq 3$, where jet rank is the number of leading jets to include in the tag. The choice depends on the physics channel of interest.

- Minimum bias (soft interaction) track rejection:

- Distance in $z$ from primary vertex, $\left|z_{P V}-z_{\text {track }}\right|<0.5 \mathrm{~cm}$.

- Long lived particle rejection:

$-0 \leq 2 \mathrm{D}$ Impact Parameter $\leq 0.25 \mathrm{~cm}$,

- Decay length ${ }^{1}, D_{j} \leq 1 \mathrm{~cm}$,

- Closest distance from track arc to jet axis, $S_{j} \leq 0.07 \mathrm{~cm}$,

- Significance of $S_{j} \leq 10$.

Distributions of the variables on which quality cuts are placed online can be seen in Fig. 3.3 for three different samples: events from two runs with different initial instantaneous luminosities and for a sample of events with

\footnotetext{
${ }^{1}$ Distance along the jet axis from the PV to the point with the closest distance between the jet and the track arc.
} 
enhanced $b$-content. As can be seen the quality cuts remove tracks with outlying values of variables, that originate from secondary interactions, long lived particles and mismeasurements.

The impact parameter of the track is calculated with an iterative approach by following the arc of the track to its closest point to the jet-axis, marked by a red dot in Fig. 3.4. The impact parameter is defined as the closest distance between the tangent from this point of the track arc to the parallel line of the tangent passing through the PV as shown in Fig. 3.4. The sign of the impact parameter is positive (negative) if the closest point of the track to the jet-axis is downstream (upstream), along the jet direction, of the primary vertex. The IP significance is defined as the ratio of the IP over the DCA error, $\sigma_{D C A}$.

Figure 3.1 shows the signed impact parameter distributions for events from runs with different initial luminosities and for a sample of events with enhanced $b$-content, normalised to unit area. The positive excess of the $b$ enhanced sample over the more symmetric distribution from the other runs is clearly visible and illustrates the discriminating power of the signed IP variable.

The probability that a track originates from the primary vertex is calculated based on a fitted resolution function $R(x)$, where $x$ is the impact parameter significance. In both Run IIa and Run IIb, a combination of a Gaussian and exponential terms were used for $R(x)$ :

$$
R(x)=c_{0} e^{-0.5 \frac{x^{2}}{\sigma^{2}}}+c_{1} e^{-\frac{x}{\lambda_{1}}}+c_{2} e^{-\frac{x}{\lambda_{2}}} .
$$



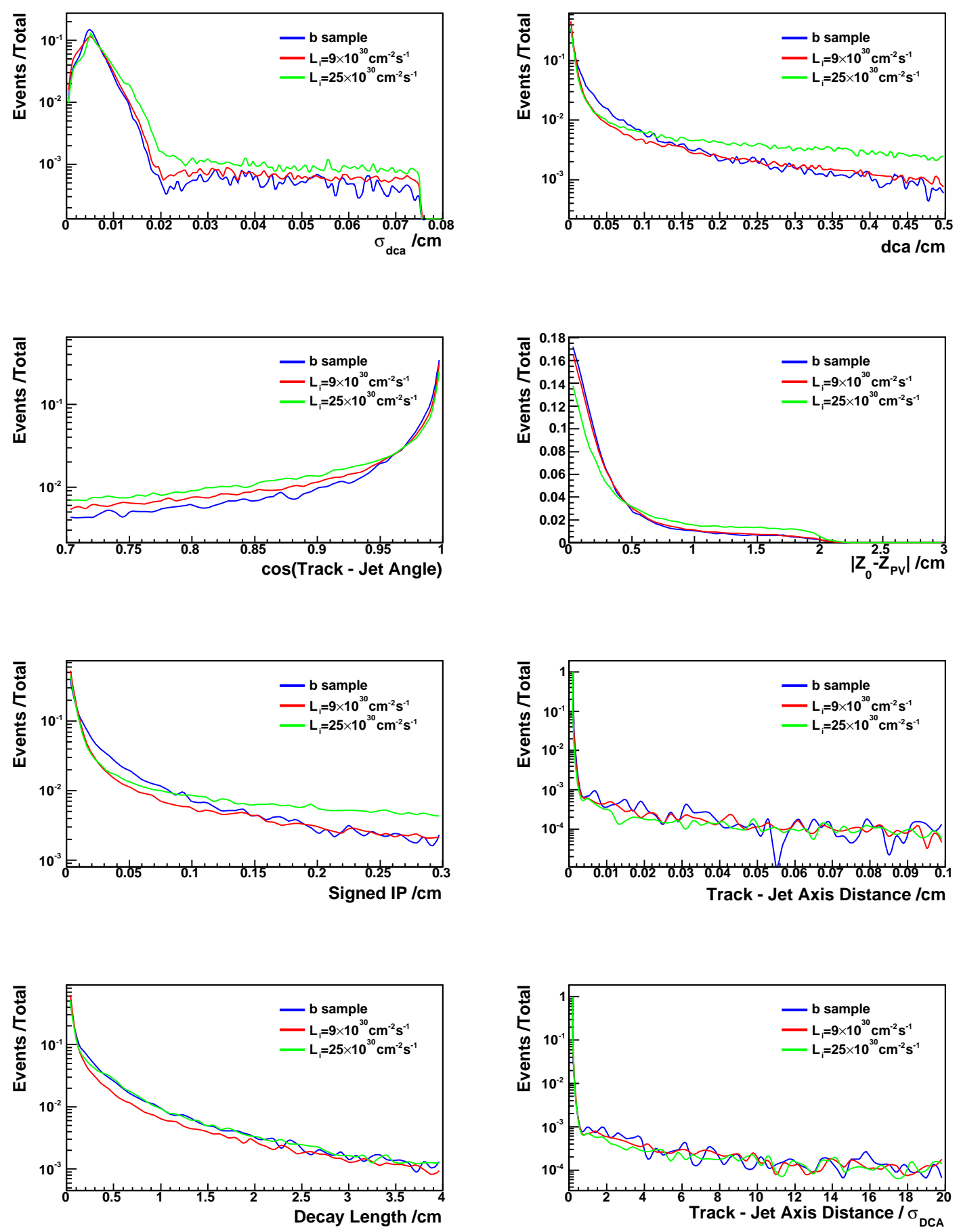

Figure 3.3: Normalised distributions of variables on which online tool cuts are placed for events from runs with different initial luminosities and for a sample of events with enhanced $b$-content. Details about the sample selection can be found in section 3.7 . 


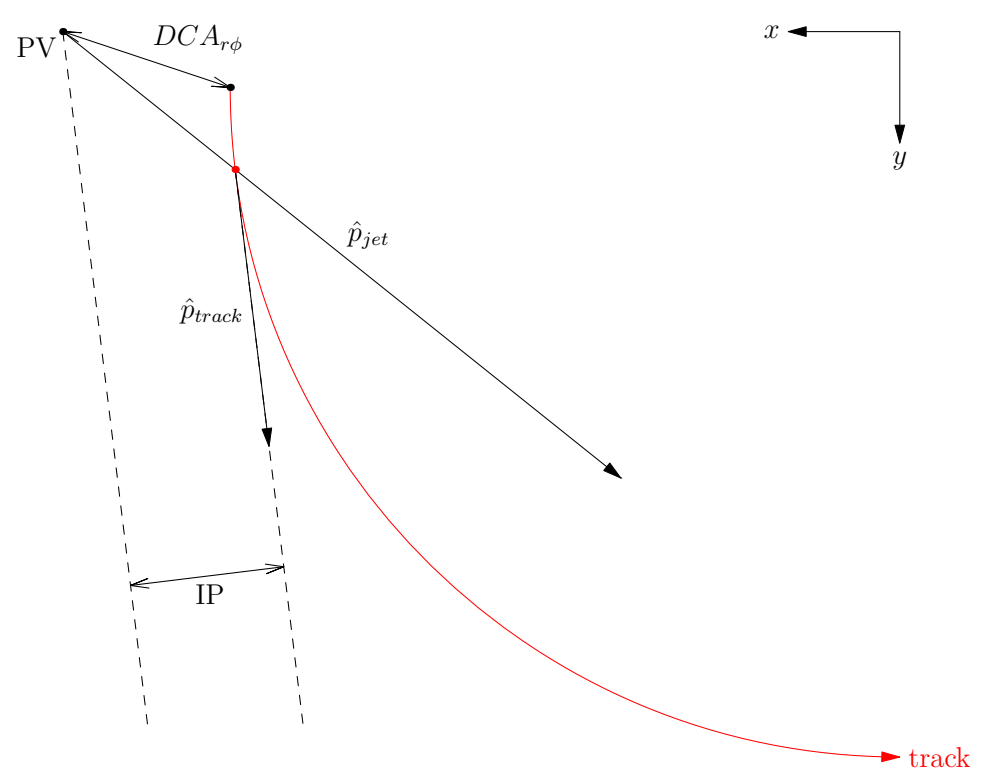

Figure 3.4: Track parameters of the Level-3 Impact Parameter calculation algorithm.

The parameters in $R(x)$ are obtained from a fit to the negative side of the signed impact parameter significance for a light-jet dominated data sample. The track probability is then calculated according to:

$$
P_{\text {track }}(x)=\frac{\int_{x}^{+\infty} R(x) d x}{\int_{0}^{+\infty} R(x) d x} .
$$

The track probabilities are combined to form jet and event probabilities according to:

$$
P_{\text {event }}=\Pi \times \sum_{j=0}^{N_{\text {tracks }}-1} \frac{(-\ln (\Pi))^{j}}{j !}
$$

with:

$$
\Pi=\prod_{i=1}^{N_{\text {tracks }}} P_{\text {track }}^{i}
$$


where $N_{\text {tracks }}$ is the number of tracks, each with track probability $P_{\text {track}}^{i}$. Since $\Pi$ is a probability, it always has a value in the range $[0,1]$ and therefore $-\ln (\Pi)$ is a positive number.

The quantity $P_{\text {event }}$, referred to as 'the $b$-tag', is the probability that tracks in an event are consistent with the primary vertex. A $b$-tag close to zero therefore suggests the presence of one or more $b$ flavour jets in the event. A jet probability is also calculated in the same way, but is not utilised in the existing trigger decision.

\subsection{Trigger Studies Samples and Tools}

Studies of the D $\varnothing$ trigger on data and Monte Carlo simulated events are carried out using an offline trigger simulation framework called trigsim [31]. To minimise the utilsation of computing resources by reducing file sizes, the standard data and MC samples used by physics analyses only contain standardised reconstructed physics objects, and the raw detector readout is removed. However, for studies concerning trigger tools, the raw detector readout is necessary, and as the standard samples cannot be used, alternative samples containing the raw readout have to be made.

Also, due to the complicated environment of a hadron collider significant data/MC differences occur when simulating the trigger [22]. Jets in MC generated events have a softer $p_{T}$ spectrum, are fewer in number and contain fewer tracks and the track errors are also not exactly reproduced. As a result, b-tagging appears to be less efficient in data than in MC. The results of tests using MC samples are thus unreliable and trigger studies are required to be 
data based.

On the other hand, purely data-driven studies avoid the problems with the simulation but are less straight-forward because the true jet flavour information is not available. For the purposes of studying and certifying the online $b$-tagging tool it was deemed sufficient to use a $b$-enhanced data sample to test signal efficiency and standard data samples to test background acceptance.

The signal samples that can be produced from single data runs are extremely limited in size. To be able to compare the tool performance for different scenarios without the errors resulting from the small signal samples being a limiting factor, a $b$-enhanced sample that spans multiple runs is necessary.

The sparse population of signal events across large numbers of data files complicates the technical process used to generate such a large $b$-enhanced sample. Furthermore, the fact that offline code is built to only run on reconstructed events while online code can only run on raw data necessitates a two pass process.

First, event lists were generated by running the offline NN b-tagging algorithm over a large data skim requiring the presence of $b$-tagged jets. The data skim was selected using triggers that require the presence of a muon. This was done to avoid biasing the jet content of the skim by the trigger requirements and because of the relatively high percentage of $b$ jets that decay semileptonically to muons.

Due to the scarcity of signal events, each event is typically recorded on a different data storage tape. The files containing each individual event were 
accessed from central storage and the raw data for these events is concatenated to form new files that are stored on local storage. This process overcomes data access limitations posed by the tape storage system and greatly increases the processing speed.

Finally, the infrastructure for running the offline trigger simulation framework over events from disparate runs was greatly improved. For the online trigger code, parameters that are constant during each run, namely the beamspot position and the magnetic polarity of the solenoid, are retrieved from a database at the beginning of each run. This behaviour was simulated in trigsim which required run-specific parameters to be set manually and assumed their values to be constant over all events processed. An addition to the framework was developed, based on earlier work [22], that automatically updates the relevant information for each event, allowing us to process files that contain concatenated events from different runs.

There was continuous development of the Level 3 tools during the long Tevatron shutdown between Run IIa and Run IIb to take the new running conditions into account. In particular, the online tracking algorithm went through a number of iterations to implement the added SMT Layer 0 and to improve timing performance and maintain efficiency for higher instantaneous luminosities. The IP significance had to be parametrised with each major reworking of the tracking algorithm. Considerable effort went into producing, testing and commissioning each parametrisation, often using limited datasets and in short time intervals, in order to maintain high trigger uptime. In this chapter, the IP significance parametrisation corresponding to the final iteration of the tracking is presented and evaluated. It is not foreseen that 
any significant modifications will be made to the Level-3 algorithms for the rest of the lifetime of the Tevatron and it is therefore expected that the parametrisation presented here will be used to collect the majority of the data recorded by the $\mathrm{D} \varnothing$ detector.

\subsection{IP Significance Fit}

The fit to the negative IP significance for a light-jet dominated data sample that was used to parametrise the L3 lifetime probability distribution function for Run IIb is shown in Fig. 3.5. Events from a run with instantaneous luminosity $L_{i}=0.9-0.7 \times 10^{31} \mathrm{~cm}^{-2} \mathrm{~s}^{-1}$ were used. This run was selected as it covers a range of luminosities for which the SMT occupancy allows for good tracking performance and there were no problems reported for any of the components of the detector.

Events are required to have at least 2 jets, each with $p_{T}>12 \mathrm{GeV}$, and $\left|z_{P V}\right|<35 \mathrm{~cm}$, using the L3 jet and vertex finding algorithms, to mimic the trigger requirements in the $b$-tagging filter that is run online. Events for which the online tracking algorithm has failed to associate any tracks to a primary vertex (and thus returns the beamspot position as the PV) were excluded for the fit. The same function as in Run IIa (Eqn. 3.1) was used.

\subsection{Certification}

To evaluate the online IP $b$-tagging efficiency, a sample of Run IIb events with two offline NN b-tagged jets was selected randomly from a skim of 


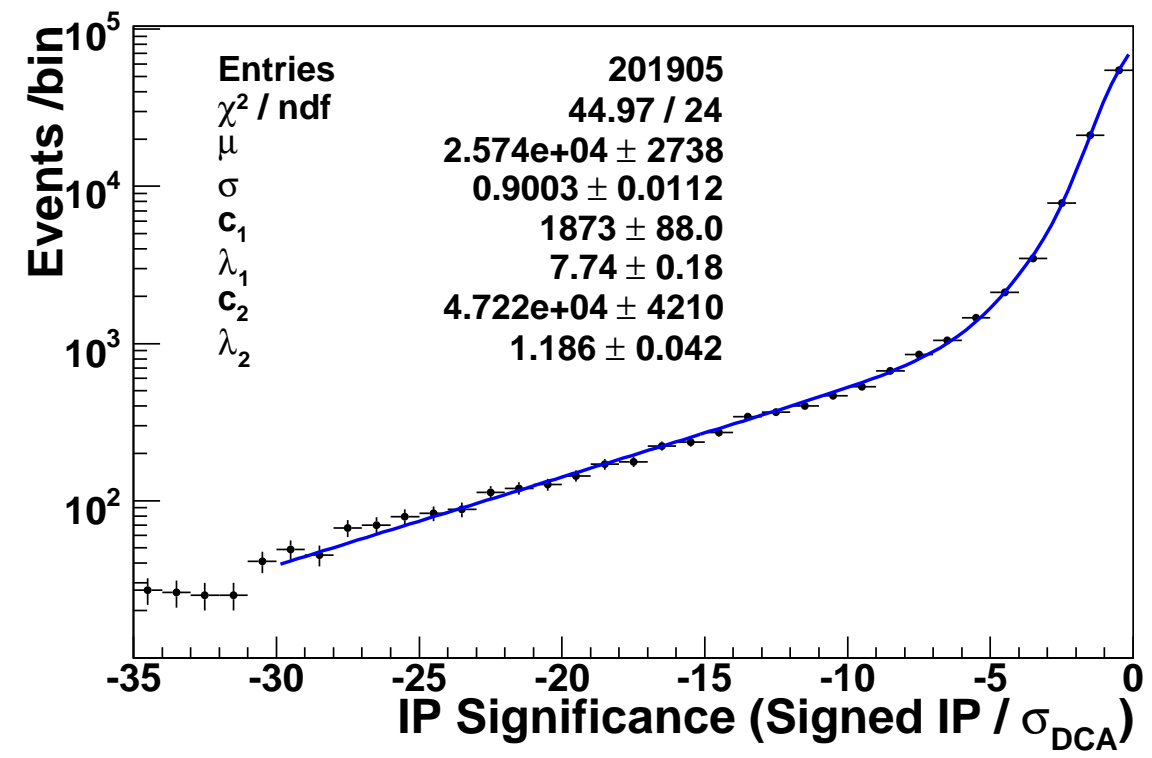

Figure 3.5: Negative IP significance for a light-jet dominated data sample (black points) fitted with the sum of a Gaussian and two exponential decay functions (blue line) in the range $[-30,0]$. 


\begin{tabular}{rccc} 
Sample Type & Run Number & $\mathcal{L}_{i} / \mathrm{cm}^{-2} \mathrm{~s}^{-1}$ & Events \\
\hline Typical $\mathcal{L}_{i}$ & 230474 & $0.9-0.7 \times 10^{31}$ & 201905 \\
High $\mathcal{L}_{i}$ & 229725 & $25-18 \times 10^{31}$ & 32973 \\
b-enhanced & $222000-232000$ & & 16242 \\
\hline
\end{tabular}

Table 3.1: Data samples used for the certification of the Run IIb Level-3 IP $b$-tagging algorithm.

\begin{tabular}{cccc} 
Flavour & Initial fraction $\%$ & Tagging efficiency $\%$ & Two tags fraction $\%$ \\
\hline b & 2.3 & 55 & 89 \\
$\mathrm{c}$ & 4.5 & 14 & 10.9 \\
udsg & 93 & 0.6 & 0.1 \\
\hline
\end{tabular}

Table 3.2: Typical sample composition for PYTHIA [32] QCD dijet MC $\left(p_{T}=\right.$ $40 \mathrm{GeV}$ ), the offline NN tagging efficiency (CC jets with $p_{T}=20 \mathrm{GeV}$ ) for the 'MEDIUM' operating point and the resulting expected composition of a $b$-enhanced sample after requiring two tags.

events that fired muon triggers over a range of luminosities. The requirement of two tags in every event enhances the purity of the sample. The sample composition before and after the $b$-tagging requirements and the efficiency in MC simulations of the $b$-tagging operating point chosen is shown in Table 3.2.

To evaluate the online performance, events from runs with different instantaneous luminosities $\left(\mathcal{L}_{i}\right)$ were used (Table 3.1). The raw data chunks for these samples were passed through the D $\varnothing$ trigger simulation and cuts were applied to emulate the online tools that are run before the Level-3 $b$-tagging tool. To avoid any bias from the trigger, events that exclusively fired triggers with vertex or impact parameter requirements at Level-3 were excluded from the samples used for certification.

The $b$-tagging distribution for the $b$-enhanced sample (Fig. 3.6, top) peaks at very low values, and is close to zero everywhere else, as expected due to the presence of $b$-content and the high purity of the sample. The $b$-tagging 
distributions for events from other runs follow a more uniform distribution throughout their range. Significant background rejection can be achieved by a tight cut $(\sim 0.05-0.1)$ on the $b$-tagging distribution while maintaining high $b$-tagging efficiency $(\sim 80-90 \%)$ (Fig. 3.6, middle). Running at high instantaneous luminosities has relatively small impact on the performance of the tool, as indicated by the small difference in efficiency and equivalent acceptance between the runs at difference luminosities for all cuts (Fig. 3.6, bottom).

\subsection{Summary}

The trigger system is the cornerstone of a modern hadron collider experiment because of the rarity of interesting processes and the large overall data production rates. The identification of $b$-quarks at the trigger level is important for a multitude of physics channels at D $\varnothing$, including top pair, single top, and Higgs production. The evaluation of lifetime probabilities using the impact parameter information for tracks in an event is a very powerful tool. Essential maintenance of the impact parameter (IP) based $b$-tagging tool was carried out. The IP resolution function was parametrised to take into account the addition of Layer 0 in the Silicon Microstrip Tracker, improvements in the tracking algorithm and the record instantaneous luminosities achieved in Run IIb of the Tevatron.

The Run IIb certified version of the IP $b$-tagging tool is expected to be run online throughout the life expectancy of the D $\varnothing$ experiment and with current projections will amass approximately $90 \%$ of the total data 

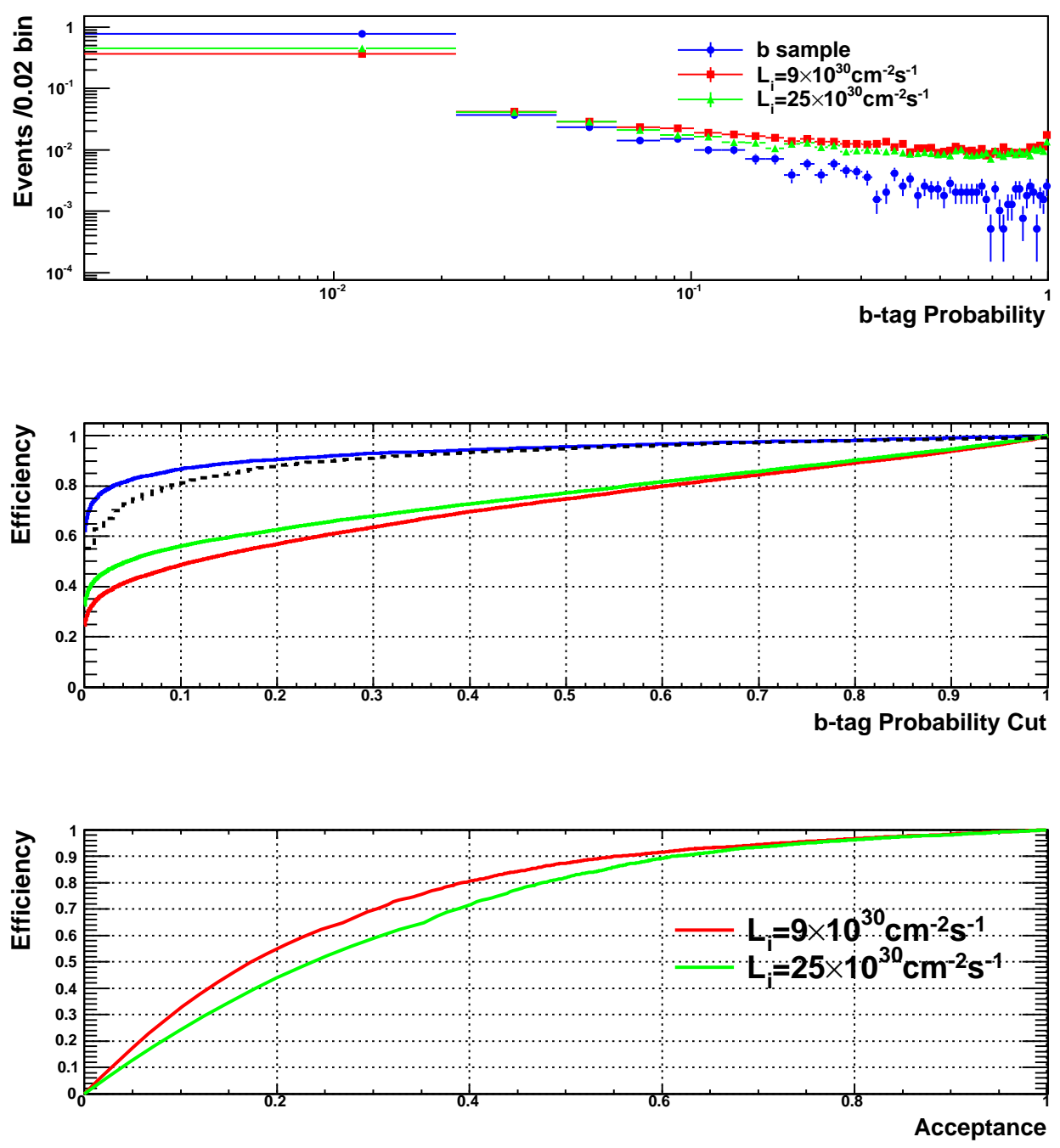

Figure 3.6: Normalised combined lifetime $b$-tagging probability distributions for events from Run IIb runs with different instantaneous luminosities $\left(\mathcal{L}_{i}\right)$ and for a sample of events with exactly two jets and two offline NN $b$-tags at the 'MEDIUM' operating point (top), the Level-3 $b$-tagging efficiency against a cut on the probability distributions (middle) and the online signal efficiency against overall acceptance (bottom). The black dotted line corresponds to the efficiency of the Run IIa parametrisation, evaluated on a sample of events with two offline SVT [22] b-tags at the 'TIGHT' operating point and is shown for reference. 
recorded with $b$-id triggers. Triggers based on the $b$-tagging tool can be used as complementary to triggers involving leptons and jet kinematics to increase the acceptance of signal processes. The impact parameter tool can be combined with a secondary vertex finder to improve the signal or to further reduce the background acceptance. 


\section{Chapter 4}

\section{Search for $Z H \rightarrow \nu \bar{\nu} b \bar{b}$ using $0.9 \mathrm{fb}^{-1}$}

\subsection{Introduction}

The search for $Z H \rightarrow \nu \bar{\nu} b \bar{b}$ at $\mathrm{D} \varnothing$ is motivated in Sec. 1.5. We look for the combined signal from $W H$ and $Z H$ decays, denoted as $V H$. There are two types of backgrounds: physical processes modeled by Monte Carlo (MC) and instrumental background predicted from data. Artificial neural networks (NN) were used for jet $b$-tagging and to classify events based on topological information from kinematic variables. We set limits on the Higgs boson cross section as a function of mass using the NN output as the discriminating variable. 


\subsection{Samples}

\subsubsection{Data}

This search used data recorded by the D $\varnothing$ detector during Run IIa, between 2002 and 2005, corresponding to trigger list versions v12-v14. Dedicated triggers selected events with acoplanar jets and large imbalance in transverse momentum, as defined by energy deposited in the $\mathrm{D} \varnothing$ calorimeters. After imposing data quality requirements, the data correspond to an integrated luminosity of $0.93 \mathrm{fb}^{-1}$. Time-dependent adjustments were made to the trigger conditions to compensate for the increasing peak instantaneous luminosity of the Tevatron. The luminosity breakdown per trigger version is shown in Table 4.1.

\begin{tabular}{rccccc} 
Trigger version & v12 & v13 & v14-v14.8 & v14.8 & Total \\
\hline Luminosity $\left(\mathrm{pb}^{-1}\right)$ & 225 & 375 & 190 & 143 & 933 \\
\hline
\end{tabular}

Table 4.1: Integrated recorded luminosity $\left(\mathrm{pb}^{-1}\right)$ used in the analysis for each trigger version in Run IIa.

\section{Trigger Terms}

Two trigger terms were used in this analysis, referred to as MHT30_3CJT5 and JT1_ACO_MHT_HT. The former was used in the pre-v13 trigger list and the latter was used in trigger versions v13 and above. For the trigger, the $\not_{T}$ is calculated as the opposite of the vector sum of the $p_{T}$ of all jets and the $H_{T}$ is calculated as the scalar sum of the $p_{T}$ of all jets.

The MHT30_3CJT5 trigger term had the following selection criteria: 
L1 three calorimeter towers each with $E_{T}$ above $5 \mathrm{GeV}$.

L2 $H_{T}$ from all L2 jets in the event above $20 \mathrm{GeV}$.

L3 $\not_{T}$ from L3 jets with $p_{T}>9 \mathrm{GeV}$ above $30 \mathrm{GeV}$.

The JT1_ACO_MHT_HT trigger term had the following selection criteria:

L1 three calorimeter towers each with an $E_{T}$ above $5 \mathrm{GeV}$.

L2 $H_{T}>20 \mathrm{GeV}$; at least two jets of which the leading two are separated by $0<\phi<168.75^{\circ}$, where $\phi$ is the angle in the $x-y$ plane.

L3 at least one jet with $E_{T}>9 \mathrm{GeV} ; \phi$ between the two leading jets (if present) to be less than $170^{\circ} ; H_{T}$ to be greater than $30 \mathrm{GeV}$ and the $H_{T}$ sum greater than $50 \mathrm{GeV}$.

The absolute overall trigger efficiency for a $Z H \rightarrow \nu \bar{\nu} b \bar{b}$ (with Higgs boson mass, $m_{H}=115 \mathrm{GeV}$ ) sample was estimated to be $\sim 50 \%$, and $\sim 80 \%$ relative to basic analysis cuts $\left(\operatorname{Jet}_{1,2} p_{T}>20,15 \mathrm{GeV} ; E_{T}>35 \mathrm{GeV}\right.$ ) using simulations of the trigger [33].

A trigger simulation was used to model the effects of the trigger requirements on events generated by Monte Carlo (MC) processes (Sec. 4.3).

\subsubsection{Monte Carlo Simulations}

\section{Monte Carlo Generators}

ALPGEN [34] is a leading order generator for hard multiparton processes in hadron collisions. PYTHIA [32] contains theory and models for hard and soft interactions, parton distributions, initial- and final-state parton showers, 
multiple interactions, fragmentation and decay and it is used by $\mathrm{D} \varnothing$ to incorporate jet hadronisation and gluon radiation by initial or final state partons. MCFM (Monte Carlo for FeMtobarn processes) [35] is a Monte Carlo simulator designed to calculate cross-sections for various femtobarn-level processes at hadron-hadron colliders. For most processes, matrix elements are included at next-to-leading order (NLO) and incorporate full spin correlations.

\section{Samples Generated}

Signal samples of $Z H \rightarrow \nu \nu b \bar{b}$ and $W H \rightarrow \ell \nu_{\ell} b \bar{b}(\ell=e, \mu, \tau)$ were generated for $105 \leq m_{H} \leq 135 \mathrm{GeV}$ in $10 \mathrm{GeV}$ increments using PYTHIA version $6.232[32]$. A list of signal samples used at each mass point, the number of events generated and the expected cross section $(\sigma)$ can be seen in Table 4.2 .

A list of all the Monte Carlo generated background samples can be seen in Table 4.3. ALPGEN [34] version 2.05 was used to simulate $t \bar{t}$ production with up to four jets. Samples of $W+$ jets ( $W$ decays to all three lepton pairs for light jets $j j, b \bar{b}$ and $c \bar{c}$ jets) and $Z+$ jets (including $Z \rightarrow \nu \bar{\nu}$ and $Z \rightarrow \tau^{+} \tau^{-}$ processes for $j j, b \bar{b}$ and $c \bar{c}$ jets) were also generated separately using ALPGEN. The cross sections for the samples generated with ALPGEN were obtained following the procedures detailed in [36]. Diboson processes ( $W W, W Z$ and $Z Z$ ) were generated with PYTHIA [32]. The samples generated with ALPGEN were processed through PYTHIA for showering and hadronisation.

Next-to-leading order (NLO) cross sections were used for normalising all processes (NNLO for $t \bar{t}$ ). The NLO cross sections were taken from MCFM [35]. All samples were processed through the GEANT3-based D $\varnothing$ detector simula- 


\begin{tabular}{lcl} 
Process $\left(m_{H} / \mathrm{GeV}\right)$ & Number Generated & NLO $\sigma(\mathrm{pb})$ \\
\hline$Z H \rightarrow \nu \nu b b\left(m_{H}=105\right)$ & 62250 & 0.0221 \\
$Z H \rightarrow \nu \nu b \bar{b}\left(m_{H}=115\right)$ & 63500 & 0.0152 \\
$Z H \rightarrow \nu \nu b \bar{b}\left(m_{H}=125\right)$ & 62750 & 0.0095 \\
$Z H \rightarrow \nu \nu b \bar{b}\left(m_{H}=135\right)$ & 62500 & 0.0052 \\
\hline$W H \rightarrow e \nu_{e} b b\left(m_{H}=105\right)$ & 53500 & 0.0207 \\
$W H \rightarrow e \nu_{e} b \bar{b}\left(m_{H}=115\right)$ & 51750 & 0.0139 \\
$W H \rightarrow e \nu_{e} b \bar{b}\left(m_{H}=125\right)$ & 56500 & 0.0086 \\
$W H \rightarrow e \nu_{e} b \bar{b}\left(m_{H}=135\right)$ & 57500 & 0.0046 \\
\hline$W H \rightarrow \mu \nu_{\mu} b b\left(m_{H}=105\right)$ & 54500 & 0.0207 \\
$W H \rightarrow \mu \nu_{\mu} b \bar{b}\left(m_{H}=115\right)$ & 50000 & 0.0139 \\
$W H \rightarrow \mu \nu_{\mu} b \bar{b}\left(m_{H}=125\right)$ & 57500 & 0.0086 \\
$W H \rightarrow \mu \nu_{\mu} b \bar{b}\left(m_{H}=135\right)$ & 52000 & 0.0046 \\
\hline$W H \rightarrow \tau \nu_{\tau} b \bar{b}\left(m_{H}=105\right)$ & 53250 & 0.0207 \\
$W H \rightarrow \tau \nu_{\tau} b \bar{b}\left(m_{H}=115\right)$ & 52500 & 0.0139 \\
$W H \rightarrow \tau \nu_{\tau} b \bar{b}\left(m_{H}=125\right)$ & 51000 & 0.0086 \\
$W H \rightarrow \tau \nu_{\tau} b \bar{b}\left(m_{H}=135\right)$ & 52000 & 0.0046 \\
\hline
\end{tabular}

Table 4.2: Signal Monte Carlo samples. NLO $\sigma$ are taken from [35].

\begin{tabular}{lcl} 
Process & Number Generated & $\sigma(\mathrm{pb})$ \\
\hline$\gamma / Z \rightarrow \tau \tau+0,1,2,3 l p$ & 1311750 & 198.4 \\
$\gamma / Z+2 c \rightarrow \tau \tau+2 c+0,1,2 l p$ & 125750 & 4.75 \\
$\gamma / Z+2 b \rightarrow \tau \tau+2 b+0,1,2 l p$ & 510250 & 1.57 \\
$\gamma / Z \rightarrow \nu \nu+0,1,2,3,4,5 l p$ & 1311750 & 1145.5 \\
$\gamma / Z+2 b \rightarrow \nu \nu+2 b+0,1,2 l p$ & 409250 & 9.2 \\
\hline$W \rightarrow l \nu+0,1,2,3,4,5 l p$ & 8222064 & 6290.9 \\
$W+2 c \rightarrow l \nu+2 c+0,1,2,3 l p$ & 1527026 & 130.7 \\
$W+2 b \rightarrow l \nu+2 b+0,1,2,3 l p$ & 1338253 & 32.2 \\
\hline$t \bar{t} \rightarrow l \nu+b \bar{b}+2 j+0,1,2 l p$ & 524000 & 2.96 \\
$t \bar{t} \rightarrow 2 l+2 \nu+b \bar{b}+0,1,2 l p$ & 498000 & 0.71 \\
\hline$Z Z$ & 203000 & 0.94 \\
$W Z$ & 305500 & 2.41 \\
$W W$ & 510000 & 7.97 \\
\hline
\end{tabular}

Table 4.3: Background Monte Carlo samples. lp stands for light partons and $j$ for jets from uds quarks and gluons not originating from the hard scatter. 
tion [37] and the reconstruction software.

Real events from randomly selected beam crossings were overlaid on simulated events to account for additional minimum bias interactions. The quality requirements applied on the data sample were applied on all MC samples.

\subsection{Trigger Parametrisation}

The trigger parametrisation is based on the method originally developed in $[33,38]$. The L1, L2 and L3 properties for an event are calculated starting from simulated jets.

For the Level-1 parametrisation, the probability is calculated that the number of trigger towers above a certain threshold and within a certain $\eta$ region meets the trigger requirements. The probability is parametrised for a dijet event sample collected with muon-based triggers which are assumed to be independent of the L1 trigger considered [39].

For higher trigger levels, the parametrisation takes simulated jets, smeared and corrected for resolution effects to match data, with $p_{T}>15 \mathrm{GeV}$ and calculates the probability for each jet to have been reconstructed and its equivalent energy at L2/L3. The calculation is based on the efficiency and resolution of L2 and L3 jets compared to jets in data passing the mark and pass $^{1}$ triggers 3CJT5 for v12 and 2CJT5_mp3_pf1 for the other trigger versions. These mark and pass triggers have the same L1 requirements as for the triggers used in the analysis.

For Level-2, the offline jet $p_{T}$ is corrected such that the coarse hadronic

\footnotetext{
${ }^{1}$ Mark and pass triggers record trigger information and write events to tape whether passed or not.
} 
fraction is removed and the vertex is set to be at the origin. This renders the offline parametrised jets more similar to the L2 jets.

The Level-3 simulation was developed for this analysis and then used by others $[40,41,42]$. For each offline jet, the response of the L3 online jet was simulated as follows:

- The probability that a Level-3 jet was reconstructed was determined from a fit to a $p_{T}$-dependent turn-on curve for the $\mathrm{CC}$ and $\mathrm{EC}$ regions separately.

- The offline jet direction was used for the simulated jets, as the agreement is good [33].

- After the L3 jet was reconstructed, the offline jet energy was corrected to the L3 value by sampling a Gaussian probability density, obtained by taking the ratio of energies of spatially matched online and offline jets. The L3 trigger parametrisation was derived especially for this analysis.

To test the performance of the trigger parametrisation, the leading and next-to-leading jet $p_{T}$ distributions were compared for events that passed the real and simulated trigger requirements; see Section 4.5.

\subsection{Event Selection}

Standard physics objects (eg. jets, electrons, muons) reconstructed using the p17 version of the D $\varnothing$ offline reconstruction code were used for the analysis. 


\subsubsection{Basic Selection}

After the trigger criteria are met, the basic event selection required:

- At least two jets with $p_{T}>20 \mathrm{GeV}$ and $|\eta|<1.1$ (central calorimeter) or $1.4<|\eta|<2.5$ (end calorimeters).

- The presence of a primary vertex with $\left|z_{P V}\right|<35 \mathrm{~cm}$, with at least 3 attached tracks.

- $E_{T}>50 \mathrm{GeV}$.

- $H_{T}<240 \mathrm{GeV}$.

- No isolated leptons (electron or muon).

These cuts select the basic topology of the events and require a well reconstructed vertex to maximise the b-tagging discrimination potential.

\subsubsection{Jets}

Jet reconstruction takes into account the expected calorimeter response, energy lost due to showering out of the jet cone, and energy deposited in the jet cone not associated with the jet [43]. Jet energies are not corrected for the presence of muons in the jet cone. Events with jets in the ICR $(1.1<|\eta|<1.4)$ were excluded from the search as the background is badly modeled. Attempts were made to include this region but this did not improve the expected sensitivity of the search due to the higher instrumental background rate. 
The $E_{T}$ is taken from the calorimeter value, with no muon correction and ignoring the unclustered energy in the $\mathrm{CH}$ layer of the hadronic calorimeter [44]. The $\not_{T}$ is calculated as the vector sum of the $p_{T}$ of all jets with a $p_{T}>20 \mathrm{GeV}$ within $|\eta|<2.5$. We define $\mathbb{P}_{T}$ as the negative of the vector sum of the $p_{T}$ of all tracks with at least 8 CFT hits, DCA to the primary vertex less than $2 \mathrm{~mm}$, $z$-distance from the primary vertex less than $5 \mathrm{~mm}$ and $\chi^{2} /$ d.o.f. $<4.0$.

To take detector modeling limitations into account, the default simulation output is modified in order to match the performance observed in data. Jets simulated by MC processes are recalibrated and discarded based on the behaviour observed in photon+jet samples, a process known as Jet Shifting Smearing and Removing (JSSR) [45].

\subsubsection{Leptons}

The isolated lepton cut eliminates a significant proportion of the analysed $t \bar{t}$, $Z$ (non-neutrino) and $W$ bosons with leptons in the final state.

Objects are identified as electrons if they fulfil the following criteria:

- Candidate track with $p_{T}>8 \mathrm{GeV}$.

- Fraction of energy in EM calorimeter over the total energy deposit, $f_{E M}=\frac{E_{E M}}{E_{t o t}}>0.9$

- Isolation fraction, $f_{\text {iso }}=\frac{E_{t o t}(0.4)-E_{E M}(0.2)}{E_{E M}(0.2)}>0.2$, where $E_{\text {tot }}(0.4)$ and $E_{E M}(0.2)$ are the energies inside a cone size of 0.4 and 0.2 in $\mathcal{R}$, for the sum of the hadronic and EM sections, and for the EM section alone, respectively. 
- Electron likelihood $>0.2$. The likelihood is constructed using 7 kinematic variables [46].

Objects are identified as muons if they fulfil the following criteria [47]:

- Centrally matched track with $p_{T}>8 \mathrm{GeV}$ and $\chi^{2} /$ d.o.f. $<4.0$ from the fit of the track arc to the detector hits.

- Hits in 3 muon segments (from A and BC layers).

- No cosmic origin (by applying timing requirement for scintillator hits).

The lepton criteria were set to have the analysis statistically independent to other D $\varnothing$ Higgs search channels which require the presence of leptons in the final state $[48,49,50]$, to facilitate a combination across all channels [51, 52].

\subsubsection{Instrumental Multijets}

Additional cuts were imposed to reject instrumental backgrounds, following previous work [53]:

- $\min \Delta \phi\left(E_{T}\right.$, jets $)>0.15$

- $\Delta \phi\left(\right.$ jet $_{1}$, jet $\left._{2}\right)<165^{\circ}$

- $E_{T}>-40 \times \min \Delta \phi\left(E_{T}\right.$, jets $)+80(\mathrm{GeV})$

- $\Delta \phi\left(E_{T}, \mathbb{Z}_{T}\right)<\pi / 2.0$

- $-0.1<\mathcal{A}\left(E_{T}, \not_{T}\right)<0.2$, where $\mathcal{A}\left(E_{T}, H_{T}\right) \equiv\left(E_{T}-H_{T}\right) /\left(E_{T}+\not H_{T}\right)$ 
The direction of the $E_{T}$ is obtained via a vector sum over calorimeter cells weighted by the deposited energy. These cuts take advantage of the fact that for events originating from hard processes with genuine missing transverse energy, the $H_{T}, E_{T}$ and $\mathbb{Z}_{T}$ point in the same direction and are correlated. However, dijet events in which one of the jets has been mismeasured typically have $E_{T}$ pointing along the direction of one of the jets. Instrumental effects produce events that tend to have $E_{T}$ and $\mathbb{T}_{T}$ misaligned (Fig. 4.2).

\section{5 $W+$ jets Control Sample}

A $W+$ jets sample was selected to test the trigger parametrisation; it has the same basic cuts applied as listed in Section 4.4.1, but in addition an isolated muon (with $p_{T} \geq 20 \mathrm{GeV}$ ) is required (as opposed to vetoing such

events), together with $\not_{T} \geq 20 \mathrm{GeV}$, which is consistent with $W$ production. This sample has no overlap with the one used in the main analysis and has virtually no multijet/instrumental background content.

This sample tests that the variables affected by the trigger are well modeled in the simulation and hence that the trigger parametrisation does indeed replicate both trigger resolutions and turn-on effects. Distributions of the variables tested are shown in Fig. 4.1. There is reasonable agreement of the shapes of the distributions. The MC expectation is normalised to the number of events observed in data. 

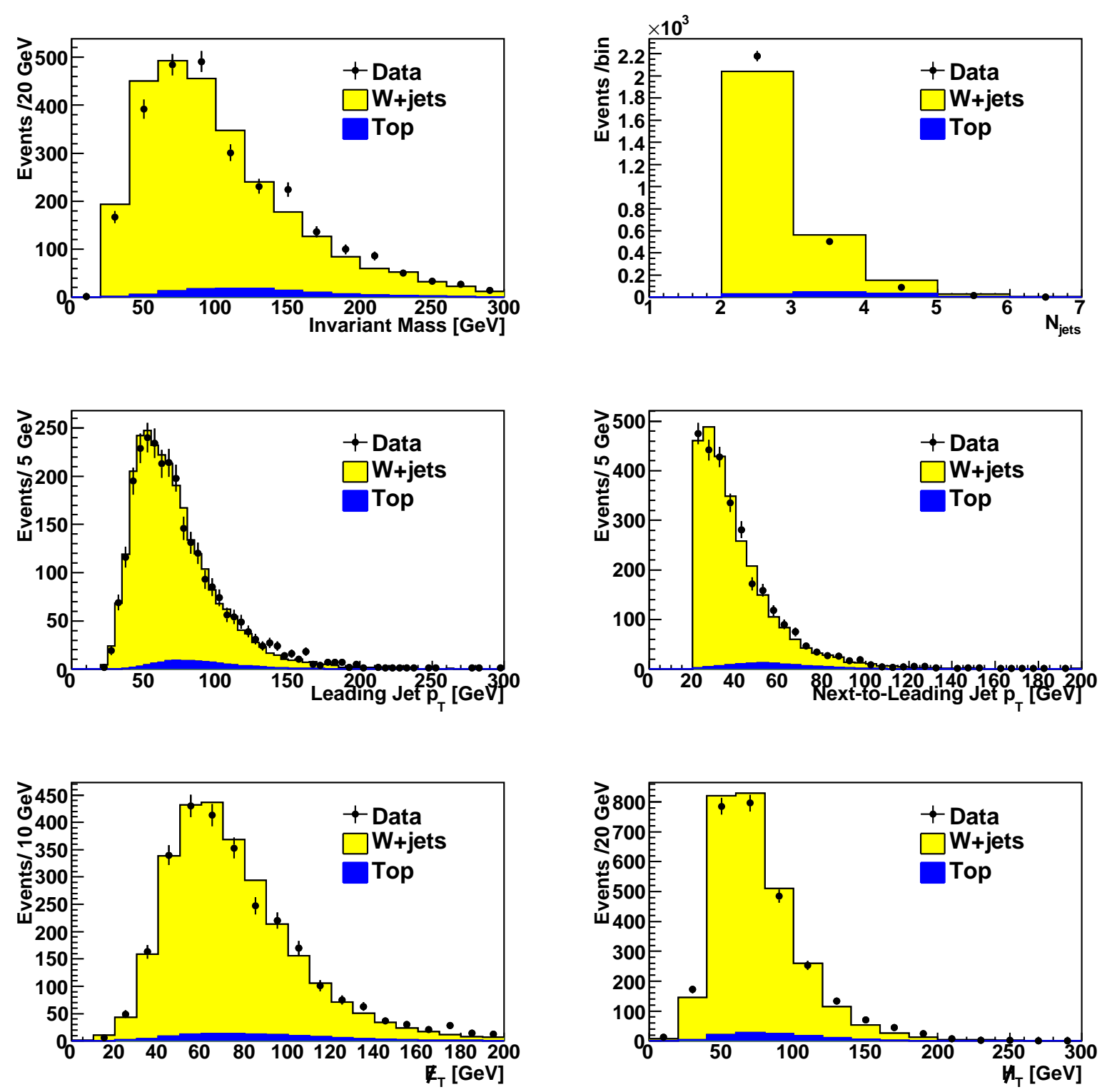

Figure 4.1: Distributions of the dijet invariant mass, the number of jets, leading and next-to-leading jet $p_{T}, E_{T}$ and $\not_{T}$ in the $W+$ jets control sample. 


\subsection{Normalisation of the Backgrounds}

The instrumental background normalisation is estimated by selecting a region of parameter space (the sideband region) which is expected to be dominated by background and have very little signal. The parameter space chosen was $\Delta \phi\left(E_{T}, \mathbb{Z}_{T}\right)$, with the signal region defined as $\Delta \phi\left(E_{T}, \mathbb{Z}_{T}\right)<\pi / 2$ and the sideband as $\Delta \phi\left(E_{T}, \mathbb{Z}_{T}\right)>\pi / 2$.

The normalisation of the $\mathrm{MC}$ and instrumental backgrounds is obtained by a combined fit to the $\mathcal{A}\left(E_{T}, H_{T}\right)$ distribution before $b$-tagging. 2D scatter plots of $\mathcal{A}\left(E_{T}, H_{T}\right)$ against $\Delta \phi\left(E_{T}, \mathbb{I}_{T}\right)$ for data, signal and background are shown in Fig. 4.2.

As seen in Fig. 4.3 the instrumental background peaks at $\mathcal{A}\left(\mathbb{E}_{T}, H_{T}\right)<0$ because it is dominated by poor quality jets that are taken into account when calculating $\not_{T}$ but not $\not_{T}$. We fit a sixth-order polynomial to the $\mathcal{A}\left(E_{T}, \not_{T}\right)$ distribution in the sideband region to determine the shape of the instrumental background (after subtracting the MC background contribution) and a triple Gaussian for the signal region.

We then do a combined physics and instrumental background fit to data in the signal region, as shown in Fig. 4.3. For this combined fit, the simulation and instrumental background shapes are fixed from the fits described above, and only the absolute scale of the two types of background is allowed to float. The level of signal events ( $W H$ and $Z H$ combined) at this point is approximately 5 events dependent on Higgs mass. Since the expected $W H / Z H$ signal is quite small, the signal contribution is neglected. The normalisation of the background for simulated (MC) processes is found to 

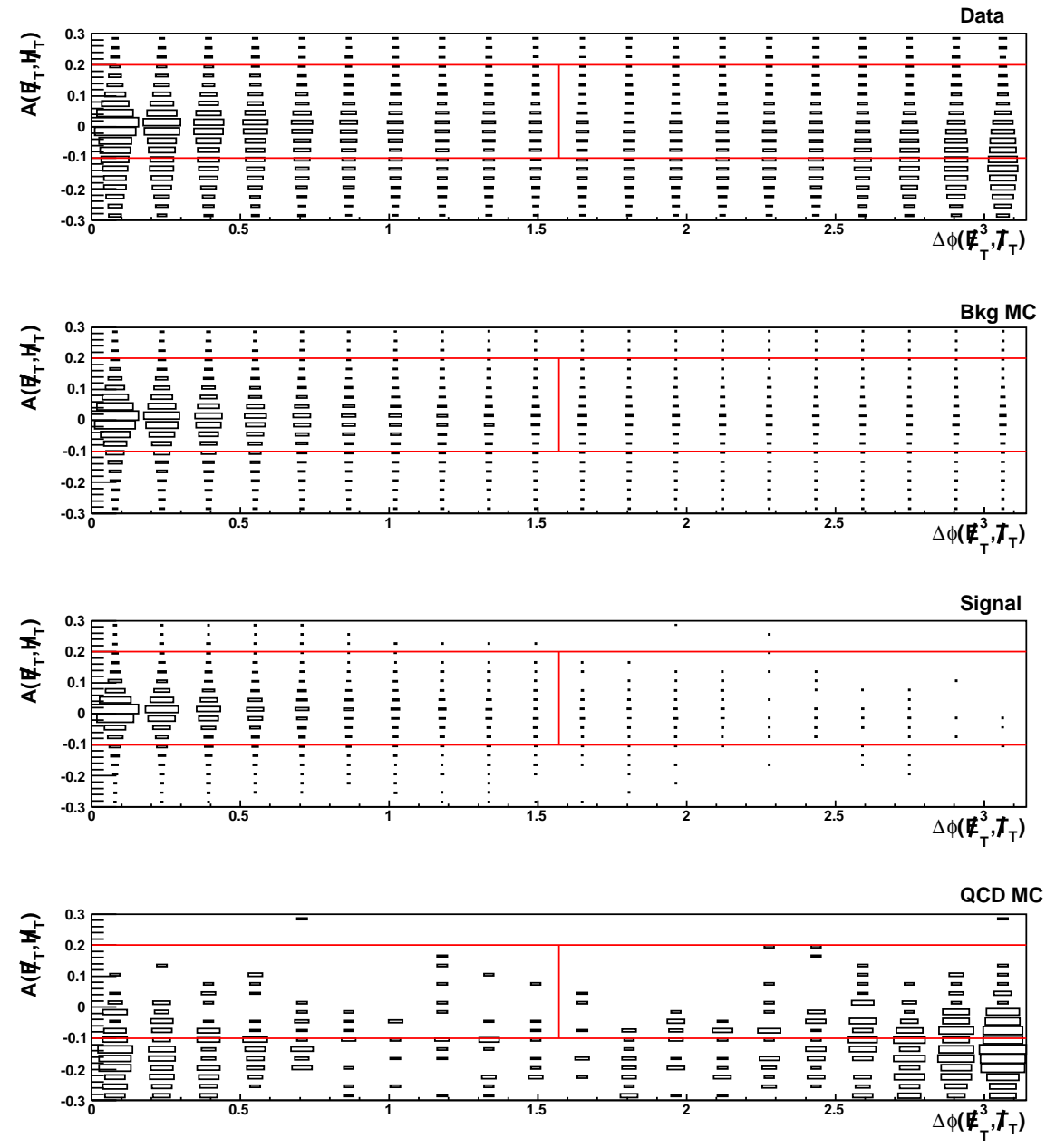

Figure 4.2: From top to bottom, 2D scatter plots of $\mathcal{A}\left(E_{T}, H_{T}\right)$ vs. $\Delta \phi\left(E_{T}, \mathbb{I}_{T}\right)$ for data, MC physics backgrounds, signal MC and dijet QCD MC $\left(20 ; p_{T} ; 200 \mathrm{GeV}\right)$. The scale of each plot is arbitrary. To retain statistics, the taggability requirement had to be relaxed for the dijet QCD MC. The red horizontal lines show the cuts placed on $\mathcal{A}\left(E_{T}, H_{T}\right)$ and the red vertical line separates the signal and sideband regions. There are significant contributions from multijet backgrounds in areas which are not populated by the physics MC. The sideband region is virtually void of signal and there is symmetry around $\Delta \phi\left(\mathbb{E}_{T}, \mathbb{I}_{T}\right)=\pi / 2$ for the QCD MC. 


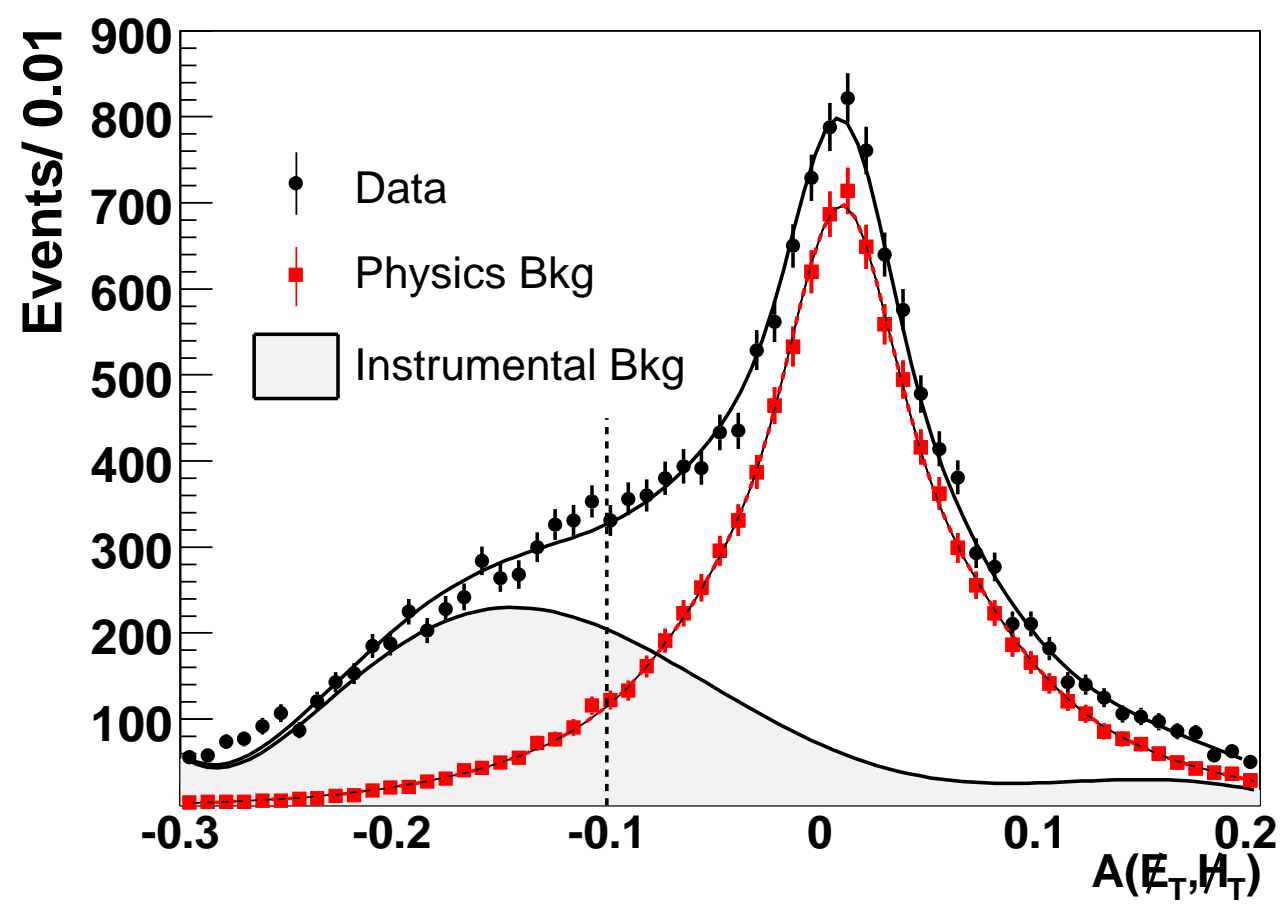

Figure 4.3: $\mathcal{A}\left(E_{T}, H_{T}\right)$ for data, MC physics background and instrumental background in the signal region, before implementing $b$-tagging. The final selection corresponds to $-0.1<\mathcal{A}\left(E_{T}, H_{T}\right)<0.2$ (indicated by the dashed line).

be $1.06 \pm 0.02$ (statistical error), in good agreement with the expected cross sections. Subsequent to the normalisation the cut $-0.1<\mathcal{A}\left(E_{T}, H_{T}\right)<0.2$ is applied to both the sideband and the signal region.

\subsection{Taggability and $b$-tagging}

\subsubsection{Introduction}

DØ uses a Neural Net to combine information from impact parameter and secondary vertex taggers, using correlations between the individual tools to 
simultaneously increase signal efficiency and background rejection [23, 54]. The NN is certified at each major production release of the $\mathrm{D} \varnothing$ offline code.

The NN is trained using MC and the efficiency and fake rate are measured on data. The tracking in the MC is not an entirely realistic simulation of data and therefore $b$-tagging is applied to MC and data differently. To accurately tag $\mathrm{MC}$ jets, tag rate functions (TRFs) and direct tag scale factors (SFs) for $b, c$, and light $/$ fake $^{2}$ jets need to be measured at various 'operating points'. An operating point (OP) corresponds to a cut on the output of a tagging tool which has a particular measured fake rate. The relevant functions are parametrised in terms of jet $p_{T}$ and $|\eta|$ and their definitions are as follows:

Scale Factor (SF) The factor by which the $b$ and $c$ MC tagging efficiencies have to be multiplied by to obtain the equivalent data tagging efficiencies.

Tag Rate Function (TRF) The efficiency to tag a jet in data. TRFs are measured separately for $b, c$ and light/fake jets. The light/fake jets TRF is also referred to as the Fake Tag Rate (FTR).

The concept of taggability is used to decouple the detector performance from the tagger performance. A "taggable" jet is expected to have the same probability of being tagged as any other taggable jet with the same $p_{T}$ and $|\eta|$, irrespective of its position in the detector.

\footnotetext{
${ }^{2}$ Light/fake here refers to $u, d, s$ quark and gluon jets.
} 


\subsubsection{Taggability}

For the purposes of this analysis, the two highest $p_{T}$ jets are required to be taggable, i.e. satisfy certain minimal tracking and vertexing criteria, so that b-tagging can be applied later. A jet must have at least two tracks, one with $p_{T}>1 \mathrm{GeV}$ and the other with $>0.5 \mathrm{GeV}$, each with more than one hit in the silicon vertex detector, and $\Delta \mathcal{R}($ track,jet $)<0.5$.

To correct the taggability in the simulation to that observed in data, the $W+$ jets control sample was used (see Sec. 4.5). The $W+$ jets sample is practically void of multijet/instrumental background.

The fraction of taggable jets was investigated as a function of $p_{T}, \eta$ and $z_{P V}$. The taggability of simulated jets was corrected by the ratio of taggabilities measured in data and in MC, which was found to depend only on $\eta$. Correction factors of $0.97 \pm 0.01$ and $0.95 \pm 0.03$ (statistical errors) were used for the central and end calorimeters, respectively.

\subsubsection{Operating Point Optimisation}

To select the optimal combination of NN b-tagging cut the effect of the choice of tagging cut on the sensitivity was studied. Three different scenarios were investigated: direct tagging on $\mathrm{MC}$ with no scale factors; direct tagging on $\mathrm{MC}$ with data/MC scale factors; and the same but with the inclusion of the instrumental background, parametrised from data.

Six neural network operating points were considered, sampling a broad range of neural network cuts. The cut on the b-tag NN output, measured efficiency and mistag rate for each operating point is shown in Table 4.4. 


\begin{tabular}{rcccccc} 
NN op pt & L5 & L3 & Loose & Medium & VeryTight & MegaTight \\
\hline NN cut & 0.15 & 0.25 & 0.45 & 0.65 & 0.85 & 0.95 \\
Efficiency \% & 80 & 75 & 70 & 65 & 55 & 50 \\
Mistag Rate \% & 8 & 5 & 2 & 1 & 0.25 & 0.17 \\
\hline
\end{tabular}

Table 4.4: Approximate efficiencies and fake tag (mistag) rates of NN operating points considered (for jet $p_{T} \sim 40 \mathrm{GeV}$ and inclusive $\eta$ ) from [55].

The sensitivity comparison for the different combinations was calculated

from $N_{\text {sig }} / \sqrt{N_{\text {bkgnd }}}$, with both the $Z H$ and $W H$ contributions included in the signal. The sensitivity was found to be maximised by the asymmetric VeryTight-L3 combination for the three different scenarios. The sensitivity ranged from $0.090(0.120) \pm 0.002$ for the worst combination to 0.125 (1.155) \pm 0.002 for the best combination with (without) the inclusion of the instrumental background, when data/MC scale factors were taken into account.

\subsection{4 b-tagging}

$b$-tagging was applied directly to the data and to the instrumental background contribution using the L3 and VeryTight operating points as determined in Sec. 4.7.2. In the simulation, direct tagging (with a relevant data/MC scale factor) was used for all jets aside from light jets. The light jet expectation after $b$-tagging was estimated by scaling the pre $b$-tag distributions by the official tag rate functions determined by the $b$-id group, parametrised in jet $p_{T}$ and $\eta[55]$. 


\begin{tabular}{lcc} 
Sample & No $b$-tag & With $b$-tag \\
\hline$Z H\left(M_{H}=115 \mathrm{GeV}\right)$ & $2.46 \pm 0.34$ & $0.88 \pm 0.12$ \\
$W H\left(M_{H}=115 \mathrm{GeV}\right)$ & $1.75 \pm 0.25$ & $0.61 \pm 0.08$ \\
\hline$W j j$ & $5180 \pm 670$ & $7.6 \pm 1.4$ \\
$W b \bar{b}$ & $397 \pm 52$ & $35.4 \pm 7.1$ \\
$W c \bar{c}$ & $1170 \pm 150$ & $9.3 \pm 1.9$ \\
\hline$Z\left(\rightarrow \tau^{+} \tau^{-}\right) j j$ & $107 \pm 14$ & $0.25 \pm 0.05$ \\
$Z(\rightarrow \nu \bar{\nu}) j j$ & $2130 \pm 280$ & $0.63 \pm 0.12$ \\
$Z\left(\rightarrow \tau^{+} \tau^{-}\right) b \bar{b}$ & $6.39 \pm 0.83$ & $0.63 \pm 0.13$ \\
$Z(\rightarrow \nu \bar{\nu}) b \bar{b}$ & $229 \pm 30$ & $24.9 \pm 5.0$ \\
$Z\left(\rightarrow \tau^{+} \tau^{-}\right) c \bar{c}$ & $12.8 \pm 1.7$ & $0.18 \pm 0.04$ \\
$Z(\rightarrow \nu \bar{\nu}) c \bar{c}$ & $467 \pm 61$ & $4.9 \pm 1.0$ \\
\hline$t \bar{t}$ & $172 \pm 34$ & $29.1 \pm 6.1$ \\
Diboson & $228 \pm 25$ & $3.84 \pm 0.50$ \\
\hline Total MC Bkg & $10100 \pm 750$ & $117 \pm 17$ \\
Instrumental Bkg & $2560 \pm 330$ & $17.2 \pm 3.4$ \\
\hline Total Bkg & $12700 \pm 800$ & $134 \pm 18$ \\
\hline Observed Events & 12500 & 140 \\
\hline
\end{tabular}

Table 4.5: Number of events after selections.

\subsection{Final Selection}

The number of events after final selections can be seen in Table 4.8. There is good agreement between the total expected and observed number of events both before and after $b$-tagging is applied. The expected and observed number of jets and dijet invariant mass distributions in the final selection before and after applying $b$-tagging can be seen in Figs. 4.4 and 4.5 respectively. 

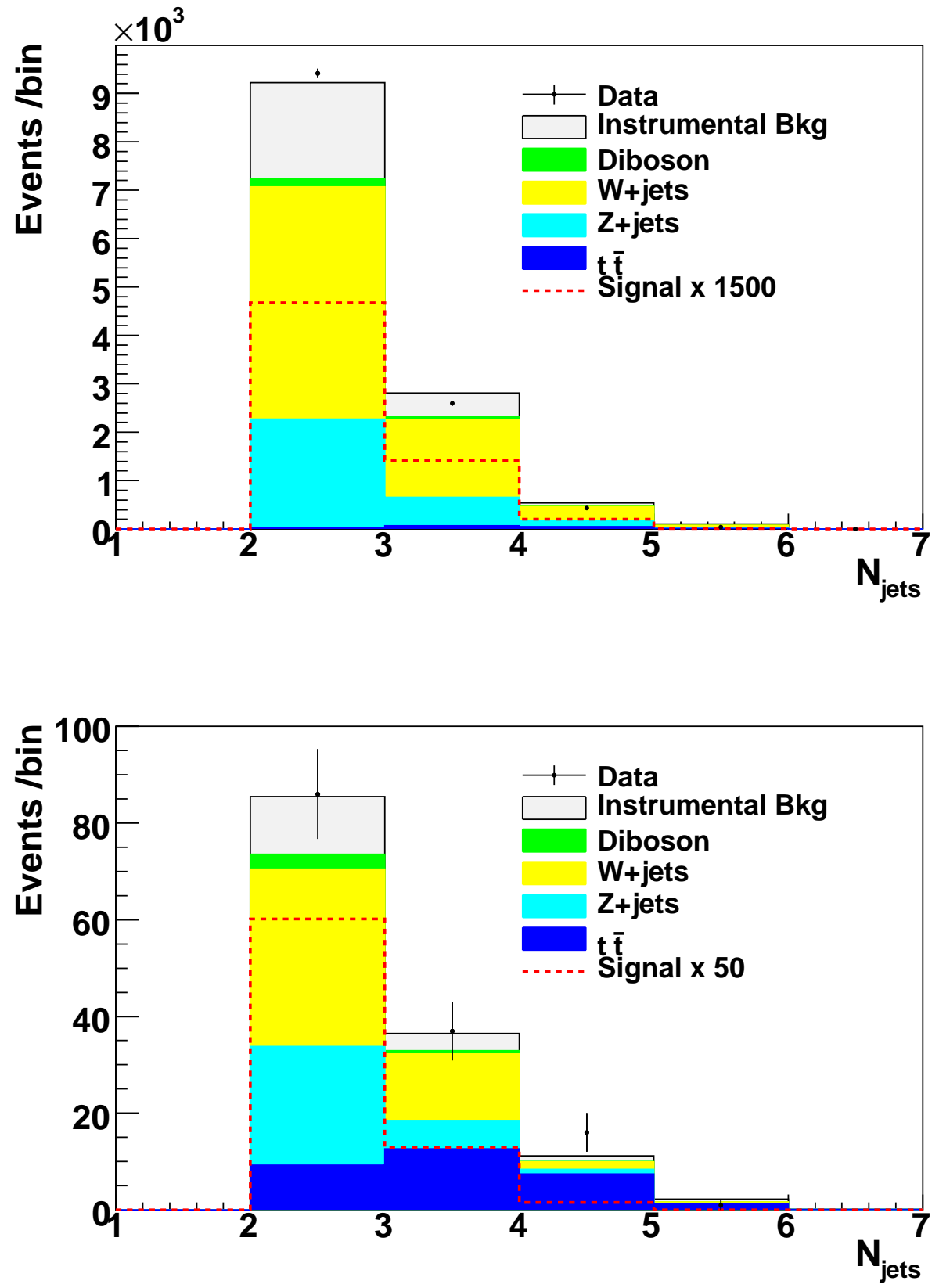

Figure 4.4: The number of jets before (top) and after (bottom) b-tagging. The signal expectation shown is for $m_{H}=115 \mathrm{GeV}$. 

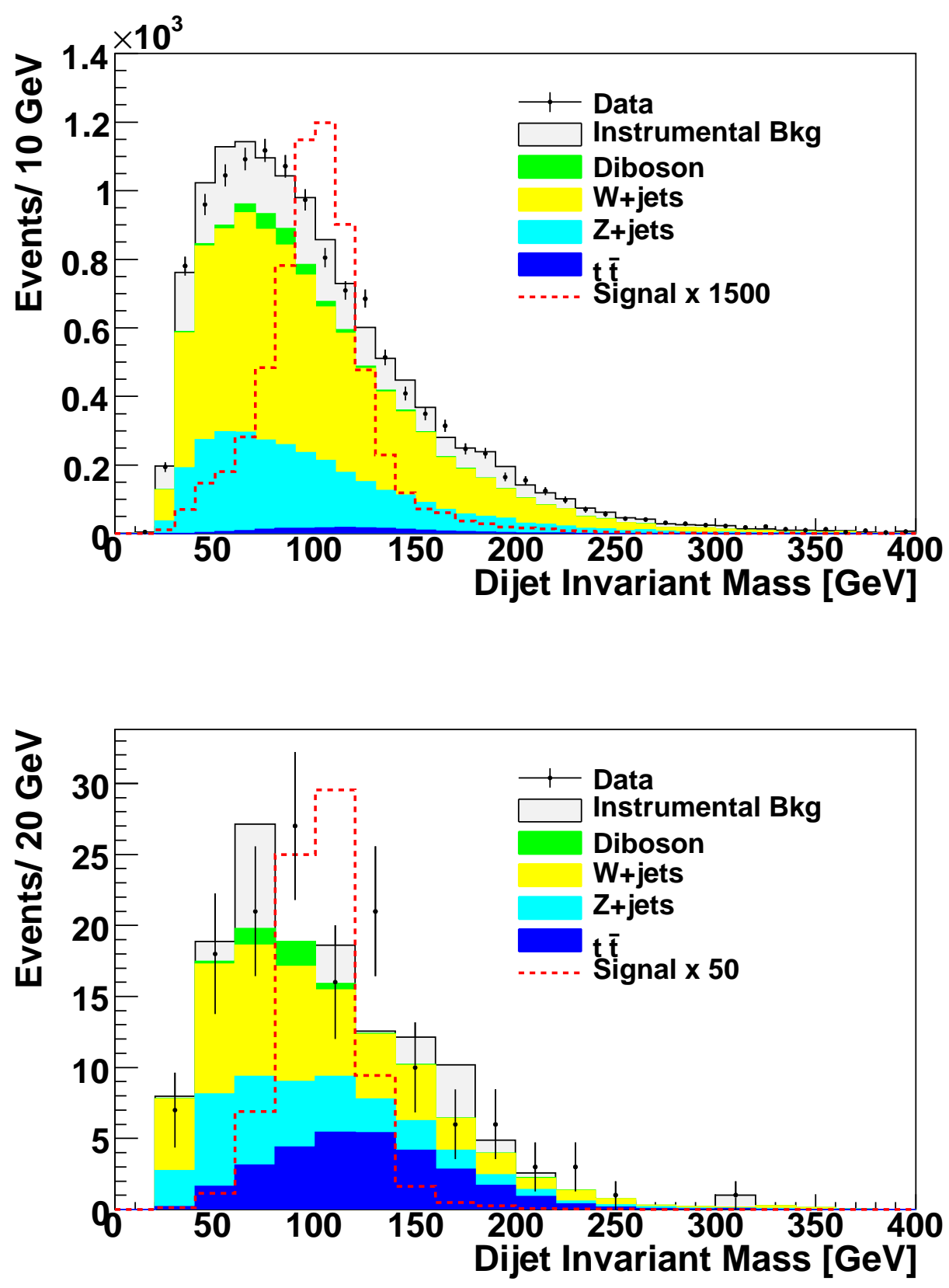

Figure 4.5: The invariant mass of the two leading jets before (top) and after (bottom) $b$-tagging. The signal expectation shown is for $m_{H}=115 \mathrm{GeV}$. 


\subsection{Artificial Neural Network Event Classifi- cation}

\subsubsection{Artificial Neural Networks}

A multilayer perceptron (MLP) Neural Network (NN) is a simple network which consists of a layer of input nodes, one or more layers of hidden nodes and one layer of output nodes [56]. Each node, called a neuron, is connected to each of the nodes in the consecutive layers by links called synapses that have a weight, $w_{j}$, and bias, $w_{0}$, representing the strength of the signal between them. The neuron, $j$, of the hidden or output layer computes a linear combination, $x_{j}$, of the neurons in the previous layer, $y_{i}$, with a bias.

$$
x_{j}=w_{0 j}+\sum w_{i j} y i
$$

The output, $y_{j}$, of the neuron $j$ is then a function of the input $x_{j}$. The function is either linear

$$
y_{j}=x_{j}
$$

or a sigmoid function

$$
y_{j}=\frac{1}{1+e^{-x_{j}}}
$$

depending on which layer is processing the input. The different layers carry out the following operations:

An input node: Receives its input from the scaled external sample and outputs to the nodes of the first hidden layer. 
A hidden layer: Outputs a sigmoid function of a linear combination of the inputs from the nodes in the previous layer.

An output layer: Creates a linear combination of the inputs from the nodes in the previous layer which is then an output of the NN.

The NN is therefore a linear combination of sigmoid functions, and it is constructed in such a way as to take advantage of two very important theorems involving the computation of linear combinations of sigmoids:

1. A linear combination of sigmoid functions can approximate any continuous function [57].

2. When trained with a desired output of 1 for signal and 0 for background, the approximate function of the input is the probability of the signal knowing the input [58].

A learning method is used to minimise the total error on a set of weighted examples. The error, known as the sample error, is defined as the sum in quadrature, divided by two, of the error on each individual output neuron. The algorithms used are based on back-propagation of the errors [59]. A training loop over all examples is called an epoch.

\subsubsection{NN Variable Selection}

A set of 7 variables was used as input to the NN. They are the invariant mass of the two leading jets in the event (Fig. 4.5), the $\Delta R$ between the two jets, the $p_{T}$ of the leading jet, the $p_{T}$ of the next-to-leading jet, the $\not_{T}$, the $\not_{T}$, and the $H_{T}$. The distributions of the input variables are shown in 
Figs. 4.6, 4.6 before $b$-tagging and in Figs. 4.8, 4.9 after $b$-tagging is applied. These input variables were selected based on their impact on the total error reduction, i.e. their ability to separate signal and background.

Variables based on tracking were excluded because of the signal/sideband region definition (see Sec. 4.6) and the use of tracking information by the $b$ tagging NN (see Sec. 4.7). Other variables tested were the centrality (defined as $\sin \theta_{1} \sin \theta_{2}$; where $\theta_{i}$ is the azimuthal angle of jet $\left.{ }_{i}\right)$, jet acoplanarity, a modified version of acoplanarity defined as $\sin \theta_{12} \cos \theta$ (where $\theta_{12}$ is the angle between the two jets and $\theta$ is the polar angle of the jet-jet system), the total $p_{T}$ of the event, the minimum acoplanarity between one of the two jets and $E_{T}$, and the acoplanarity between the $E_{T}$ and the vector sum of the two jets. Their impact on the total error minimisation did not justify their use to improve the separation between signal and background.

\subsubsection{NN Training Procedure}

To train the NN on a sample that is as close to the expectation after $b$-tagging but also be able to retain statistics, all MC in the NN training sample was used, scaled by the $b$-tagging tag rate functions for the asymmetric operating point combination chosen. The TRFs were determined on independent samples by the DØ b-id group [55]. The NN was not trained on a multijet/instrumental multijet background as it was shown that training with a pre- $b$-tag instrumental background sample had no significant impact on the performance of the NN. To evaluate the performance of the NN, the output was scanned and the maximum sensitivity obtained when placing a cut 

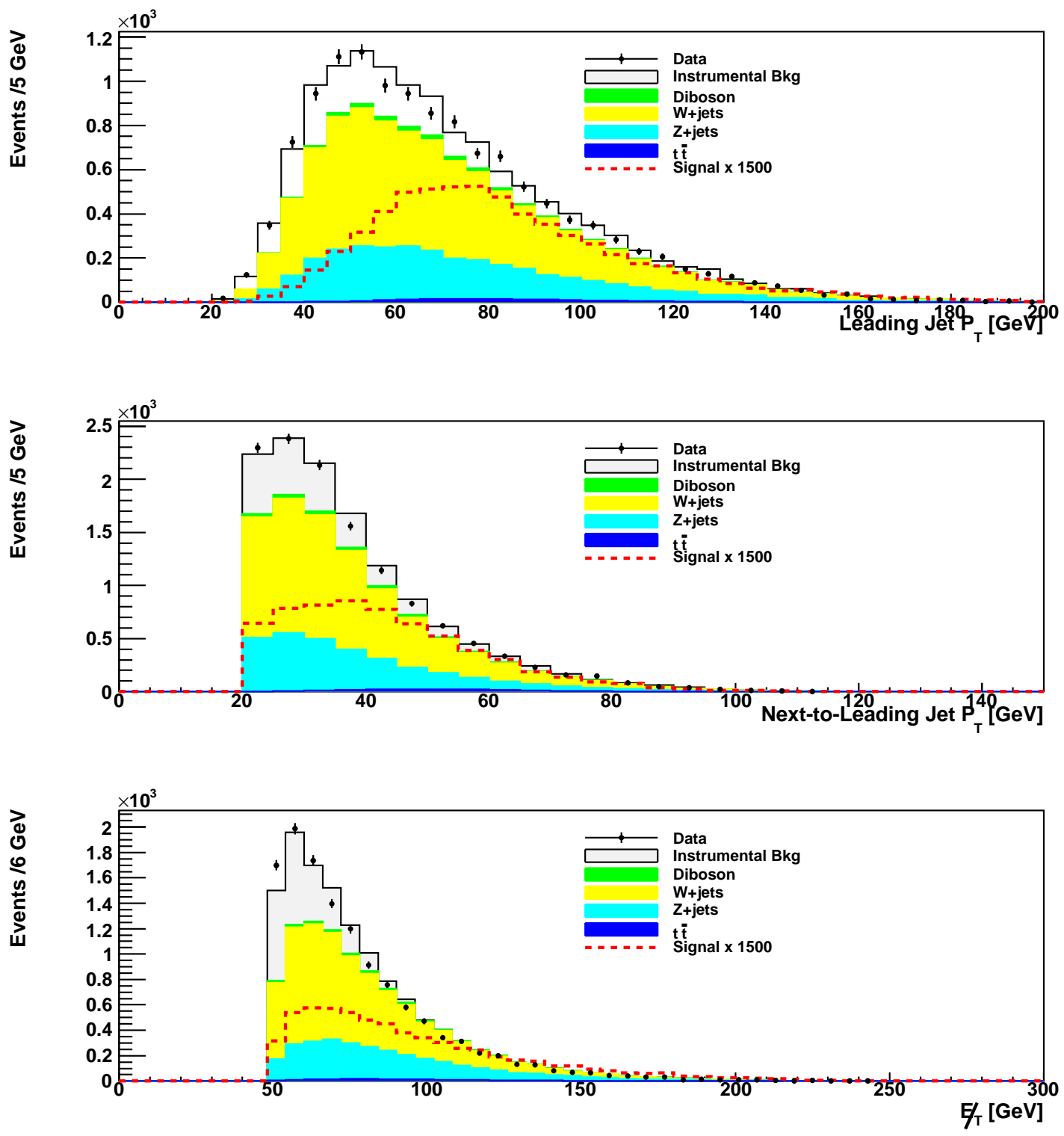

Figure 4.6: Distributions of NN input variables after final selection and before $b$-tagging is applied. The signal expectation shown is for $m_{H}=115 \mathrm{GeV}$. 

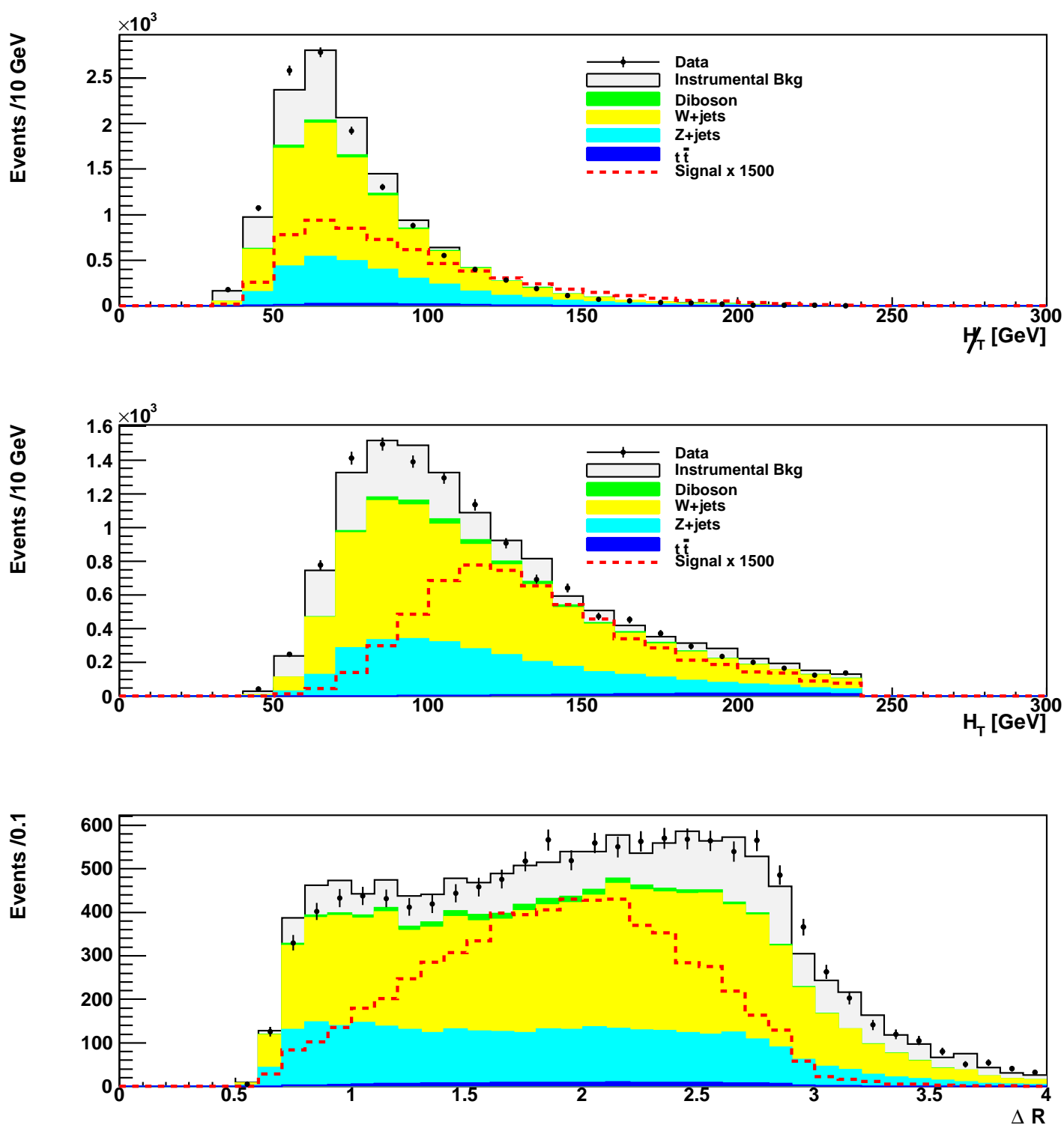

Figure 4.7: Distributions of NN input variables after final selection and before $b$-tagging is applied. The signal expectation shown is for $m_{H}=115 \mathrm{GeV}$. 

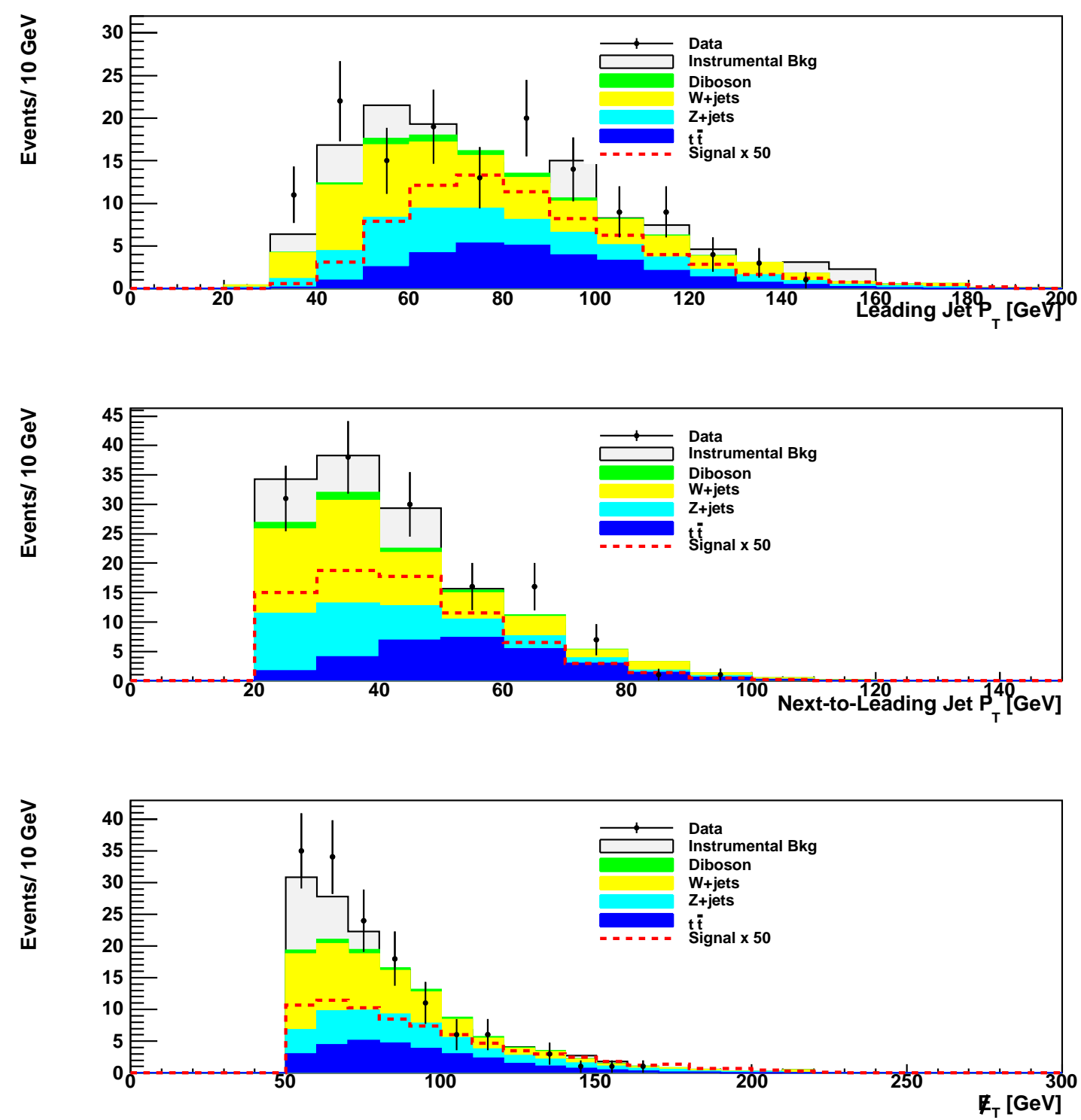

Figure 4.8: Distributions of NN input variables after final selection and $b$-tagging is applied. The signal expectation shown is for $m_{H}=115 \mathrm{GeV}$. 

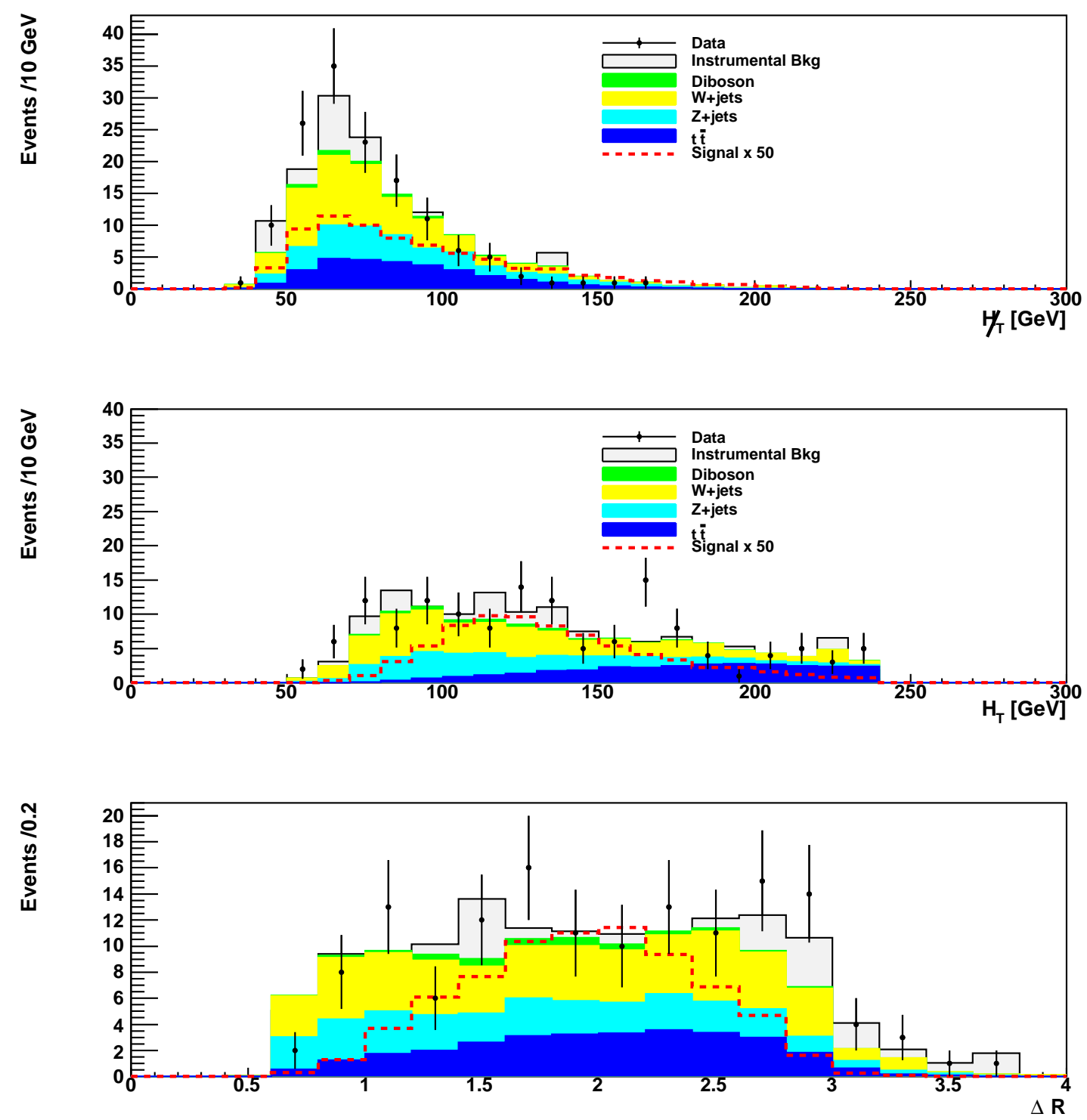

Figure 4.9: Distributions of NN input variables after final selection and $b$-tagging is applied. The signal expectation shown is for $m_{H}=115 \mathrm{GeV}$. 
$(S / \sqrt{B}$ expectation for all events above the cut) was used. The total expectation from this background amounts to $\sim 20 \%$ before and only $\sim 10 \%$ after $b$-tagging. The very limited statistics resulting from $b$-tagging this background forbid its use as a training sample. A separate NN was trained at each Higgs mass.

Different learning methods were tested for training the NN [60]:

1. Steepest descent with fixed step size (Batch)

2. Gradient steepest descent algorithm

3. The Polak-Ribiere updating formula

4. The Fletcher-Reeves updating formula

5. Broyden, Fletcher, Goldfarb, Shanno method (BFGS)

The resulting test sample error as a function of training epochs for each learning method can be seen in Fig. 4.10 (top). The BFGS learning method is chosen as it converges quickly to stability and achieves the lowest sample error. To avoid over-training, the final NN was trained for 200 epochs, as very little can be gained afterwards as seen in Fig. 4.10 (top).

The impact of the number of nodes in the hidden layer on the overall test sample error was also investigated. The test sample error is plotted against the number of nodes in the hidden layer for 200 training epochs in Fig. 4.10 (bottom). Based on this, 14 nodes were used in training and testing the final NN.

To avoid bias and over-training the NN, separate MC samples were used to train and test and to produce the final neural net output distribution used 


\begin{tabular}{cccccc} 
Luminosity & Trigger & Jet ID & $b$-tagging & Background $\sigma$ & Multijets/Instr. \\
\hline 6.1 & 5 & 5 & 7 & $6-18$ & 20 \\
\hline
\end{tabular}

Table 4.6: Overall systematic uncertainties in $\%$.

as the discriminating variable in the limit setting.

\subsubsection{NN Results}

The output of the NN shows good agreement between observed data and expectation for all Higgs masses before $b$-tagging (Fig. 4.11). After $b$-tagging, the distributions of the neural net outputs for simulated signal and background and for data events can be seen in Fig. 4.12. As expected, the background (signal) peaks at low (high) values of NN output.

\subsection{Systematic Uncertainties}

Systematic uncertainties affect the expected number of signal and background events ("overall uncertainties") as well as the shape of the distribution of the NN output ("differential uncertainties").

\subsubsection{Overall Uncertainties}

Overall uncertainties associated with the luminosity, trigger efficiencies, jet identification, $b$-tagging, $\mathrm{MC}$ and instrumental background cross sections are estimated and summarised in Table 4.6.

- The effect of applying smearing and simulated removal on the jets (Sec. 4.4.2) is calculated. The fractional difference in the number of 

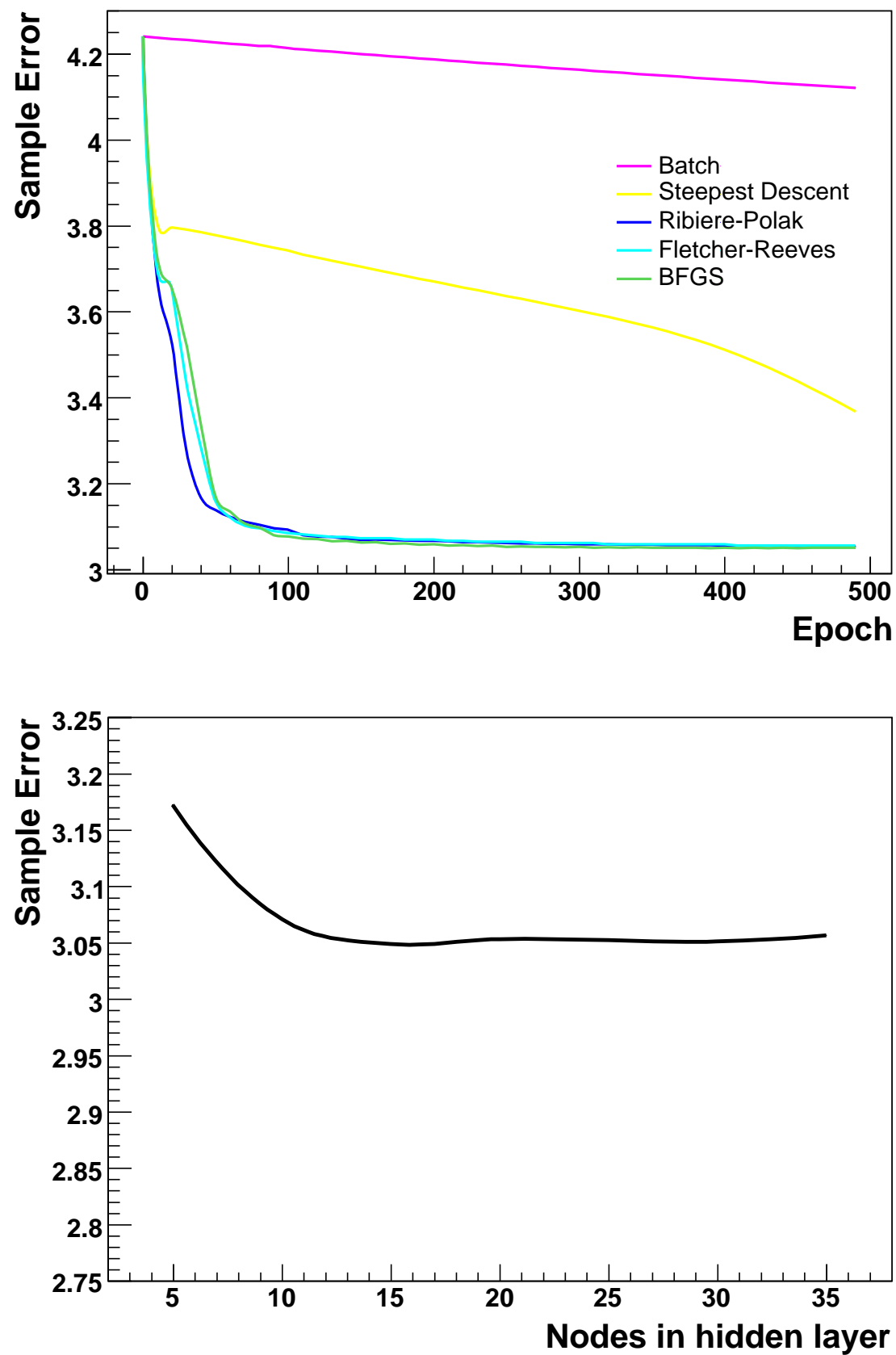

Figure 4.10: Optimisation of the NN training parameters: The test sample error against the number of training epochs for different learning methods (top) and the total test sample error against number of nodes in the hidden layer (bottom). 

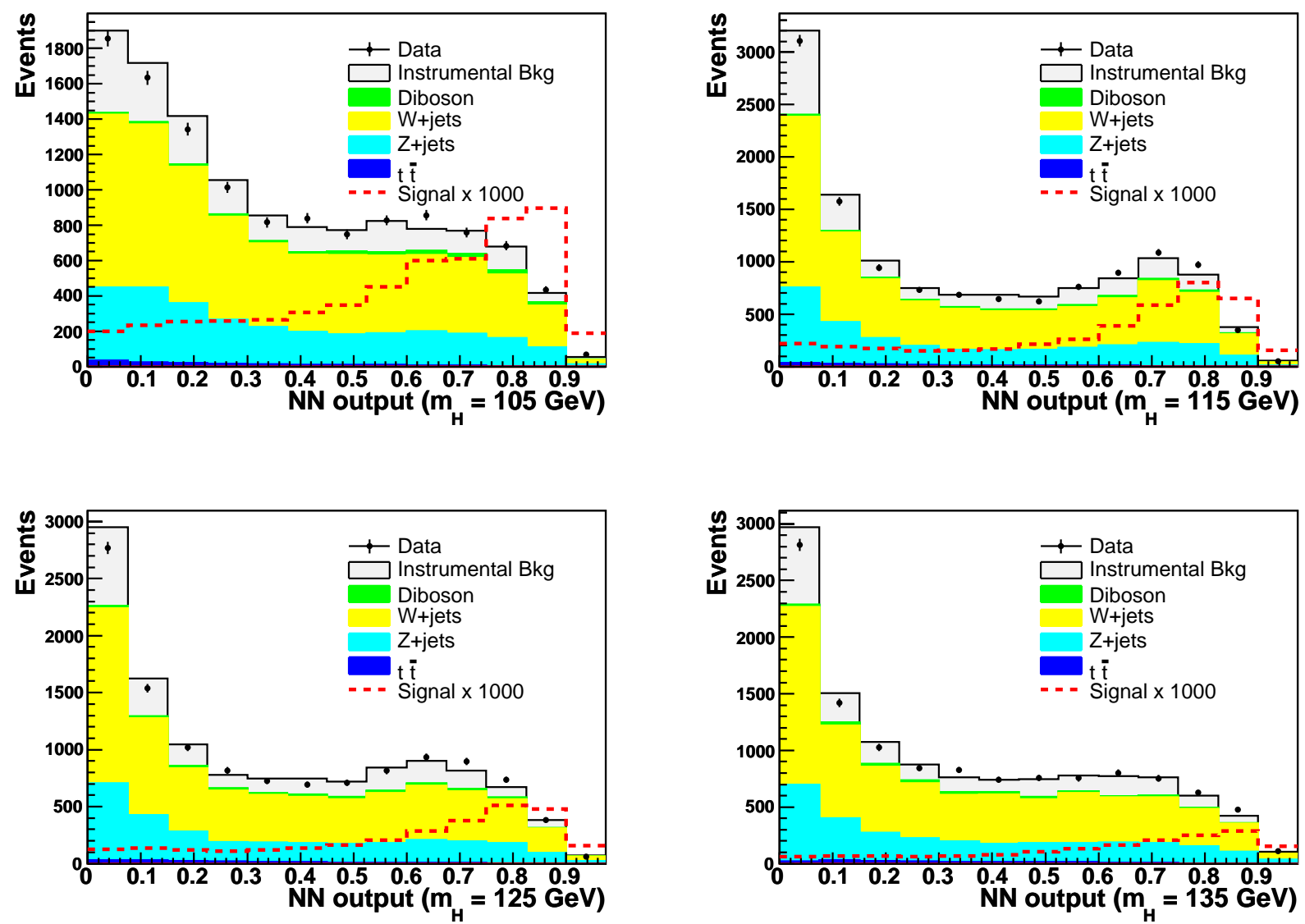

Figure 4.11: Output of the $\mathrm{NN}$ for four different Higgs masses before $b$ tagging. The signal expectation (dashed red line) is scaled by a factor of 1000 to be clearly visible. 

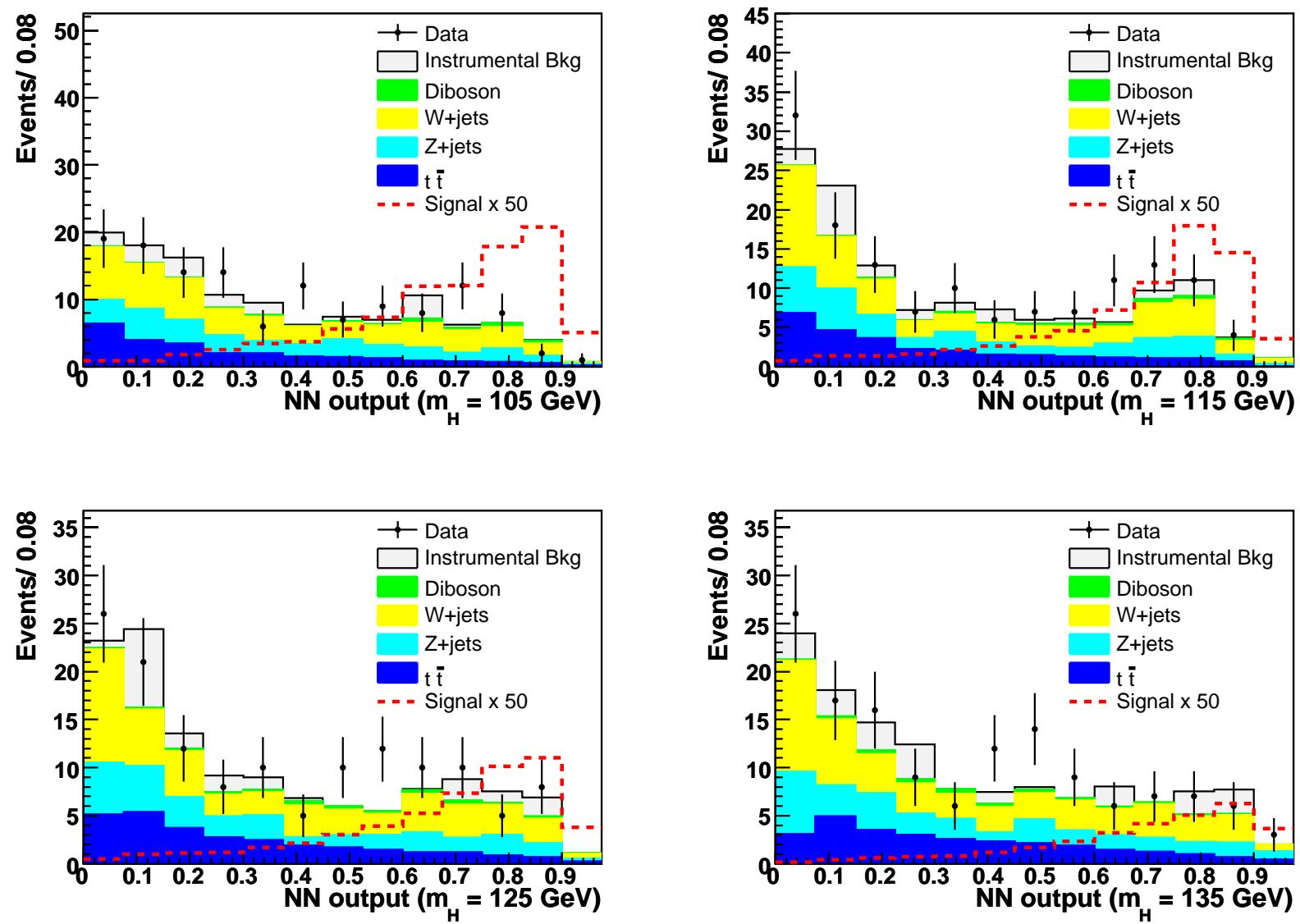

Figure 4.12: Output of the NN for four different Higgs masses after double asymmetric $b$-tagging. The signal expectation (dashed red line) is scaled by a factor of 50 to be clearly visible. 
expected events before and after shifting by one standard deviation is taken as the error.

- The $b$-tagging errors are calculated from the \pm 1 standard deviation uncertainties on the measured TRFs and scale factors used on MC and are propagated through the analysis. The same was done for the taggability uncertainty. The error quoted is a combination of the two.

- The luminosity estimation has an uncertainty of $6.1 \%$ [61].

- Systematic uncertainties are assigned on the cross sections of the various processes modeled by MC from [62]. We assigned uncertainties of $18 \%$ on the top, $15 \%$ on the $V+$ jets and $6 \%$ on the diboson cross sections.

- We use a $20 \%$ error on the rate of multijet/instrumental multijet production after $b$-tagging. This is estimated by adding in quadrature the error from the normalisation fit in Sec. 4.6 and the error on $b$-tagging the data in the sideband region. The statistical error from applying the $b$-tagging selection directly to the sideband data as opposed to using a tag rate function is propagated through the analysis and is taken into account.

- A systematic uncertainty is assigned to the residual differences between the simulated and real trigger. We compare offline variable distributions that have passed a) the online and b) the simulated triggers for data collected with mark and pass triggers. No shape dependency was observed; see Fig. 4.13. We fit the fractional difference in the $\mathbb{E}_{T}, H_{T}$ 
and dijet invariant mass separately with a constant and so derive an estimate for the error on the trigger, typically $\sim 5 \%$ as shown.

\subsubsection{Differential Uncertainties}

Differential uncertainties were estimated from the difference in the shape of the NN output:

- The impact of the jet energy scale (JES) was determined from its \pm 1 standard deviation variation propagated through the analysis in a correlated way for all signal and background samples at each mass point. This is shown in Fig. 4.14.

- The difference in the distribution of the NN output from the uncertainty in the shape of the MC di- $b$-jet mass $\left(\mathrm{m}_{b \bar{b}}\right)$ spectrum for $V+$ jets samples associated with the parameters of the generator was also taken into account [63]. This is shown in Fig. 4.15.

Additionally, the impact of the $\mathrm{MC} /$ data agreement before $b$-tagging (Figs. 4.4, 4.5, 4.6, 4.7) on the NN output was also investigated. The $m_{j j}, \Delta R$ and $H_{T}$ distributions were reweighted separately so as to show perfect agreement (in shape and absolute normalisation) between data and $\mathrm{MC}$ before b-tagging. Events were then passed through the $\mathrm{NN}$ with the new weights and the resulting $\mathrm{NN}$ output distributions were compared to the nominal ones. All differences were found to be in regions away from the signal peak and were not significant after b-tagging, as seen in Fig. 4.16. No additional 

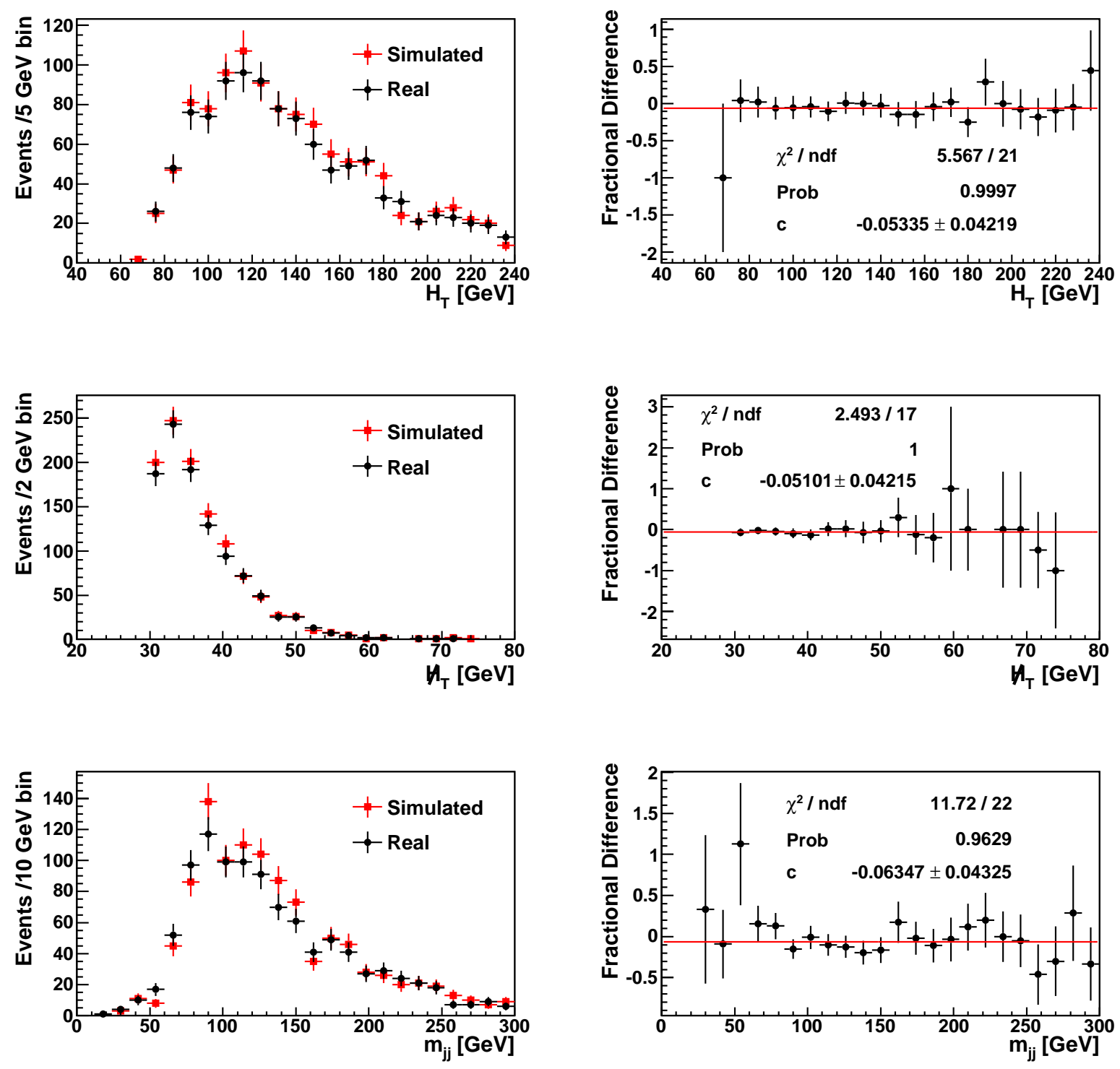

Figure 4.13: Comparison of the real and simulated trigger decisions for data events collected with mark and pass triggers (left) and fits to their fractional difference (right). 

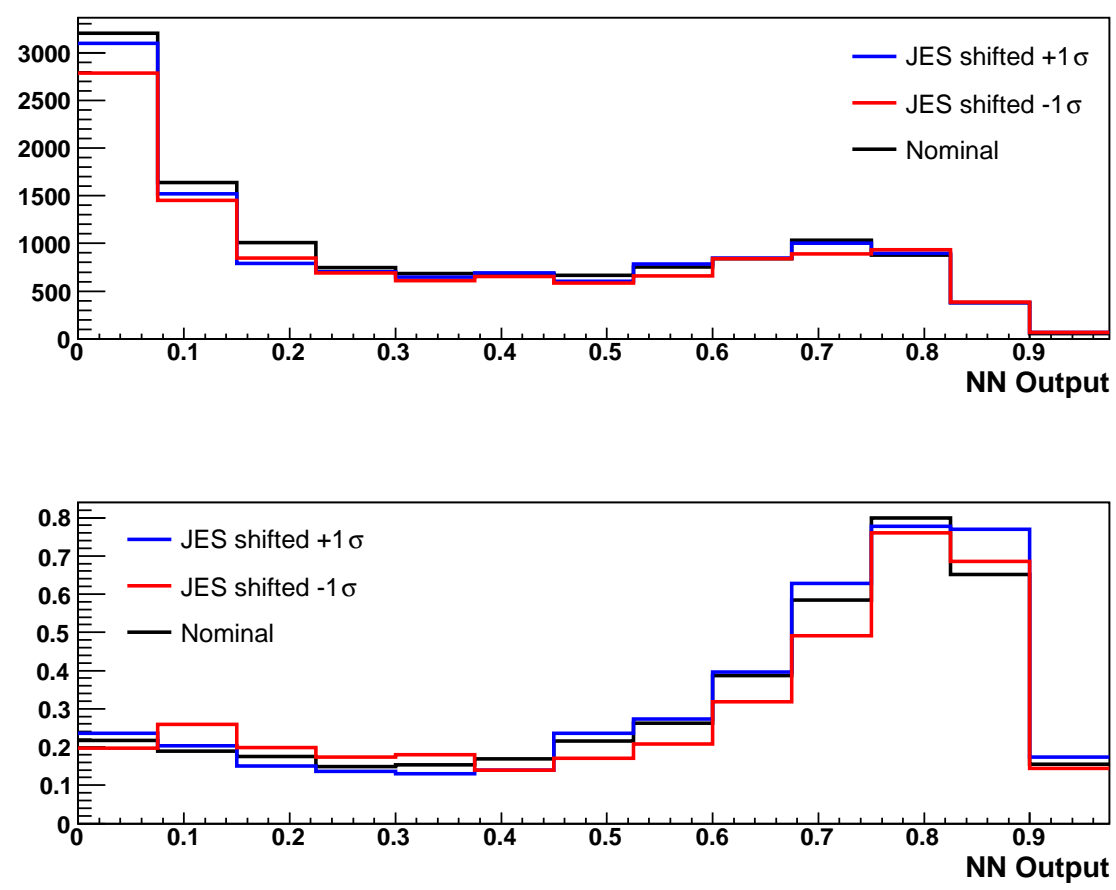

Figure 4.14: Differential uncertainty on the NN output from the \pm 1 standard deviation variation of the JES for the combined background (top) and signal (bottom) samples. 

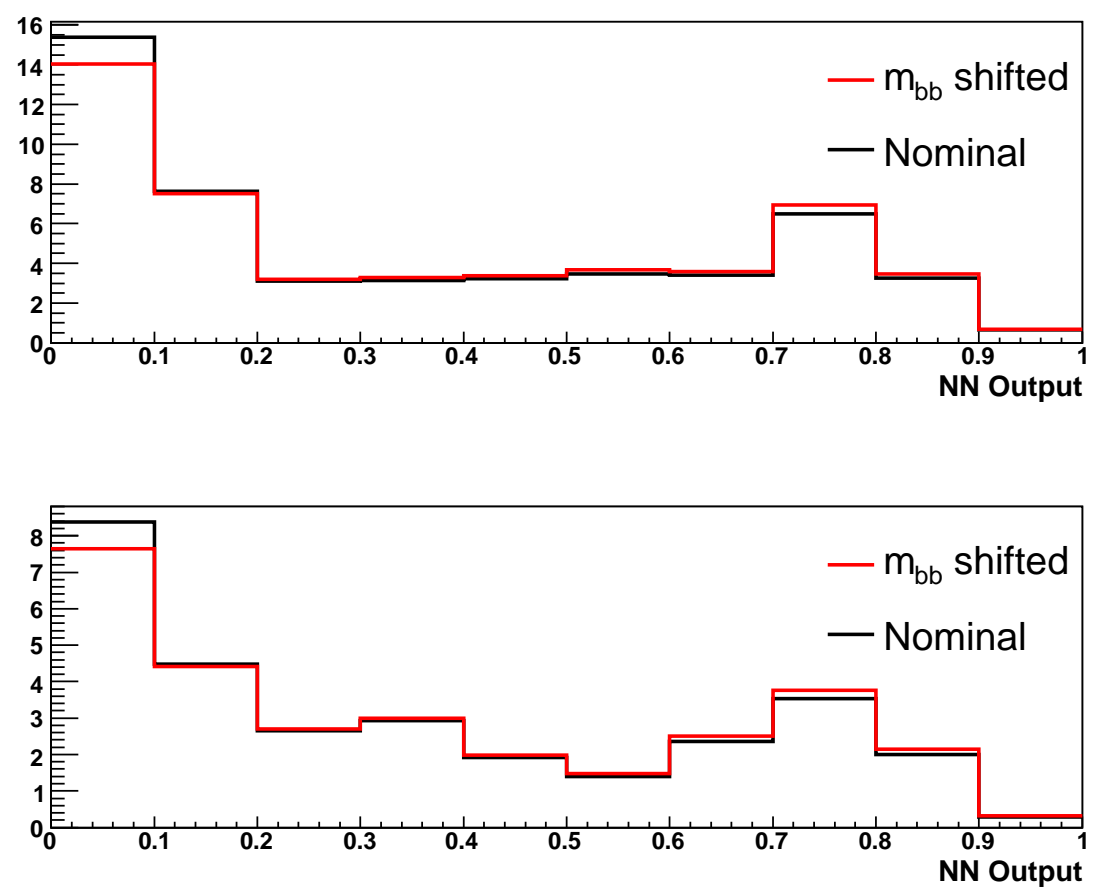

Figure 4.15: Differential uncertainty on the NN output from the uncertainty in the shape of the MC di-b-jet mass $\left(\mathrm{m}_{b \bar{b}}\right)$ spectrum associated with the parameters of the generator for $W+$ jets (top) and $Z+$ jets (bottom) samples. 

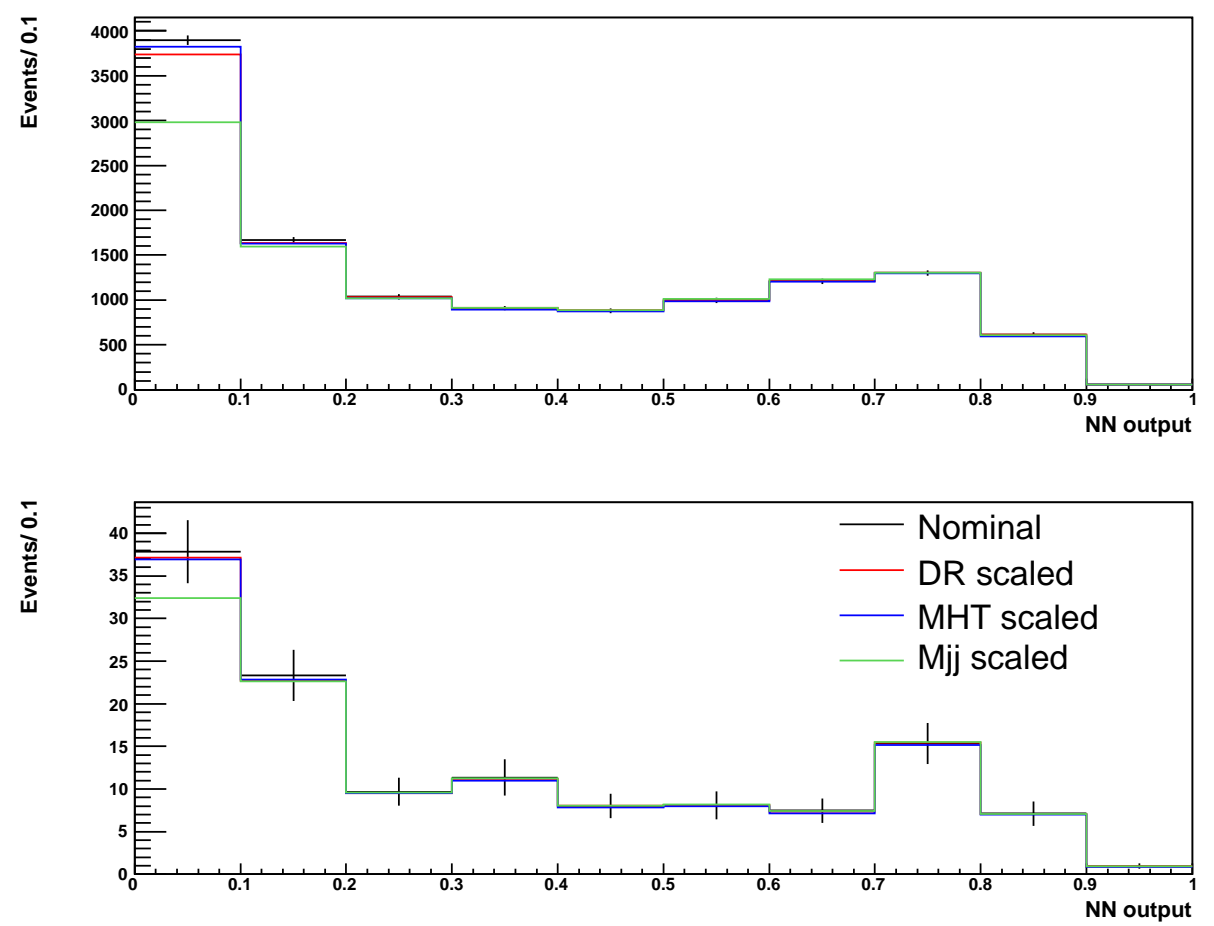

Figure 4.16: Impact of the pre-tag MC/data agreement on the NN output before (top) and after (bottom) $b$-tagging. 
systematic was thus used for the effect of the MC/data agreement before $b$ tagging. Indeed, the systematic errors included for the $m_{b \bar{b}}$ shape are larger.

All systematic uncertainties are common and correlated between signal and backgrounds, except for the uncertainties on the background cross sections and the instrumental background.

\subsection{Limits on the SM Higgs Mass}

To set limits on the SM Higgs boson production cross section, a modified frequentist approach is used [64]. The signal confidence level $C L_{S}$, defined as the ratio of the confidence level for the signal-plus-background hypothesis to the background-only hypothesis

$$
C L_{S}=\frac{C L_{S+B}}{C L_{B}}
$$

is computed. $C L_{S}$ is calculated by integration of the distributions of a test statistic over the outcomes of pseudo-experiments, generated according to Poisson statistics, for the two hypotheses. To make the computation more efficient the test statistic is calculated as the logarithmic ratio of Poisson likelihoods $\left(\mathrm{LLR}^{3}\right)$.

Systematic uncertainties are incorporated via Gaussian smearing of the Poisson probability distributions for signal and backgrounds within the pseudoexperiments. All correlations for signal and backgrounds are maintained. The value of the Higgs cross section is increased until $C L_{S} \leq 5 \%$ giving the

\footnotetext{
${ }^{3}$ Also known as the log-likelihood ratio.
} 
95\% exclusion limit for the signal at each mass point.

To reduce the impact of systematic uncertainties on the sensitivity of the analysis, the individual signal and background contributions are fitted to the data (and pseudo-data) for both the signal-plus-background and the background-only hypotheses independently by maximising a profile likelihood function for each hypothesis [65]. The profile likelihood is a function of the systematic uncertainties, which are given an additional Gaussian constraint around their predicted values.

There are two types of limits set, expected and observed. Expected limits are calculated using the simulated background and signal-plus-background distributions only, assuming no signal production, and indicate the potential sensitivity of the experiment. The observed limits are calculated using the data and the simulated signal-plus-background distributions, corresponding to the actual limit on signal production set from the data.

In Fig. 4.17, the LLR values for the signal-plus-background hypothesis $\left(\operatorname{LLR}_{s+b}\right)$, background-only hypothesis $\left(\operatorname{LLR}_{b}\right)$, and the observed data $\left(\operatorname{LLR}_{o b s}\right)$ are shown. The shaded bands represent the 1 and 2 standard deviation $(\sigma)$ departures for $\mathrm{LLR}_{b}$. These distributions can be interpreted as follows:

- The separation between $\mathrm{LLR}_{b}$ and $\mathrm{LLR}_{s+b}$ provides a measure of the overall power of the search. This is the ability of the analysis to discriminate between the signal-plus-background and background-only hypotheses.

- The width of the $\mathrm{LLR}_{b}$ distribution (shown here as 1 and $2 \sigma$ bands) 


\begin{tabular}{rcccc} 
Higgs Mass $(\mathrm{GeV})$ & 105 & 115 & 125 & 135 \\
\hline$Z H$ Expected & $1.6(15)$ & $1.5(19)$ & $1.4(29)$ & $1.2(47)$ \\
$Z H$ Observed & $1.5(14)$ & $1.5(20)$ & $1.4(30)$ & $1.3(51)$ \\
\hline$W H$ Expected & $4.8(25)$ & $4.3(33)$ & $3.8(47)$ & $3.6(84)$ \\
$W H$ Observed & $4.4(23)$ & $5.0(39)$ & $4.4(55)$ & $4.2(99)$ \\
\hline$V H$ Expected & $2.8(9.1)$ & $2.5(12)$ & $2.3(18)$ & $2.0(30)$ \\
$V H$ Observed & $2.6(8.7)$ & $2.7(13)$ & $2.5(20)$ & $2.3(34)$ \\
\hline
\end{tabular}

Table 4.7: Expected and observed limits in pb and as a ratio to the SM Higgs cross section (in parentheses), assuming $H \rightarrow b \bar{b}$.

provides an estimate of how sensitive the analysis is to a signal-like fluctuation in data, taking account of the presence of systematic uncertainties. When, for example, a $1 \sigma$ background fluctuation is large compared to the signal expectation, the analysis sensitivity is limited.

- The value of $\mathrm{LLR}_{\text {obs }}$ relative to $\mathrm{LLR}_{s+b}$ and $\mathrm{LLR}_{b}$ indicates whether the data distribution appears to be more signal-like or background-like. As noted above, the significance of any departures of $\mathrm{LLR}_{\text {obs }}$ from $\mathrm{LLR}_{b}$ can be evaluated by the width of the $\mathrm{LLR}_{b}$ distribution.

The inclusion of the systematic uncertainties causes a $\sim 25 \%$ degradation of the expected limit. The expected (observed) limits on $\sigma(p \bar{p} \rightarrow V H) \times$ $B(H \rightarrow b \bar{b})$ at the $95 \%$ confidence level range from $2.8(2.6) \mathrm{pb}-2.0(2.3) \mathrm{pb}$ for Higgs boson masses in the range 105 - $135 \mathrm{GeV}$ (Table 4.7). The expected and observed limits, along with the SM prediction, are shown in Fig. 4.18 as a function of Higgs mass. 

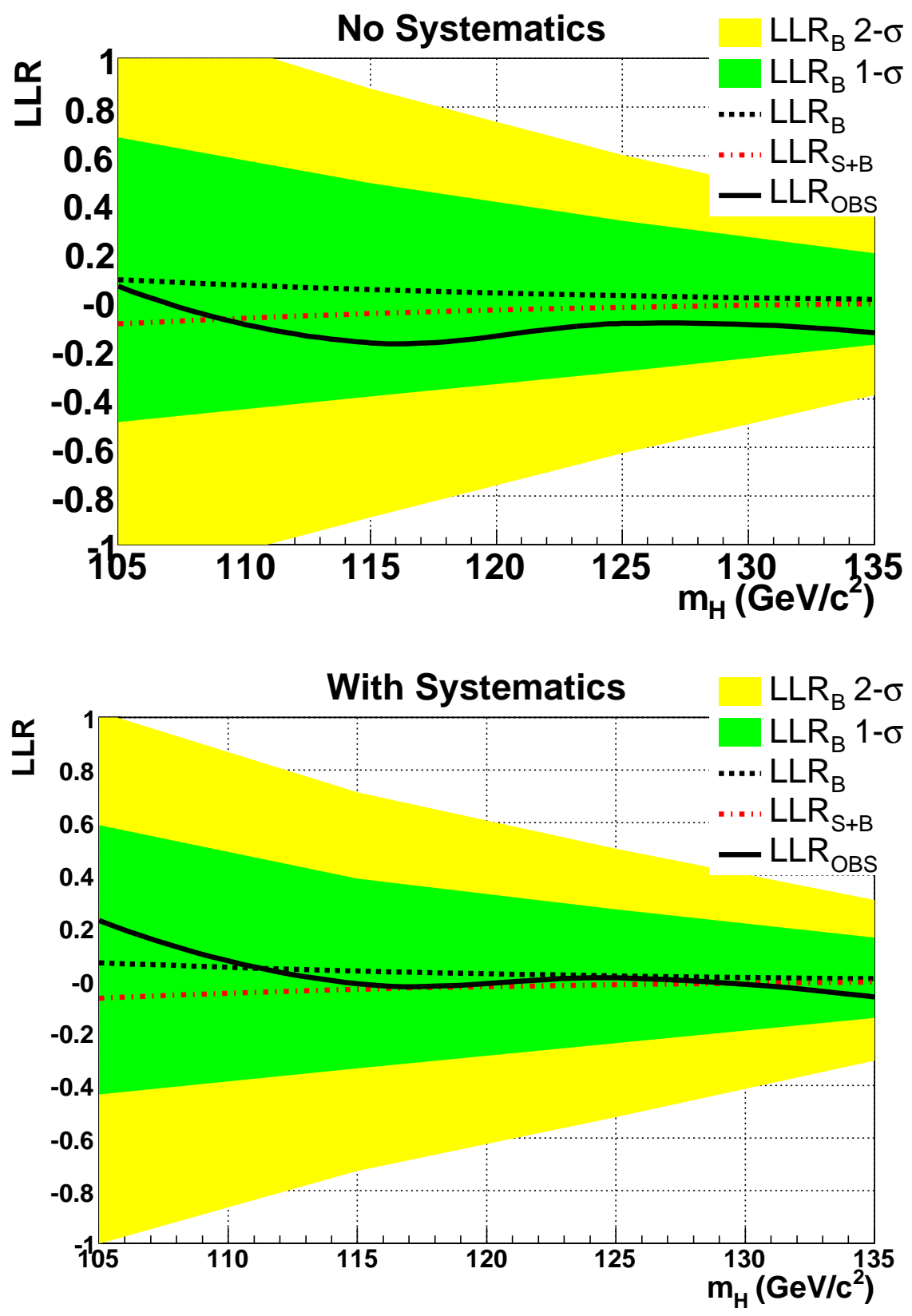


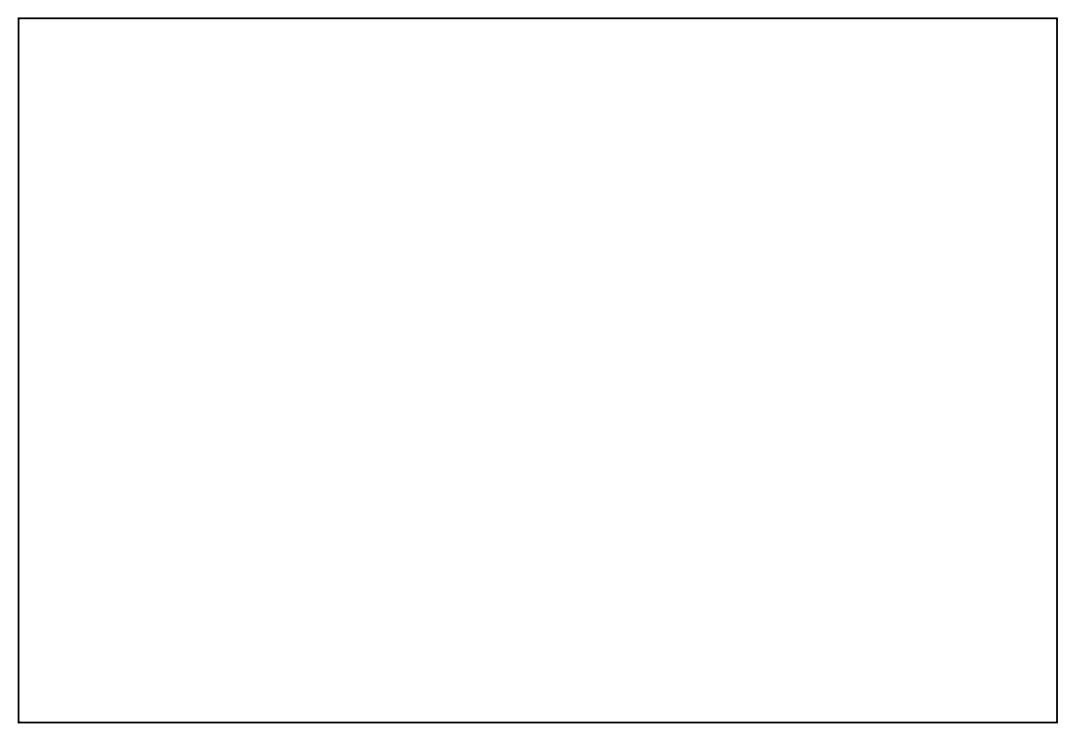




\section{Chapter 5}

\section{Evolution of the $Z H \rightarrow \nu \bar{\nu} b \bar{b}$ Analysis}

The search for the SM Higgs boson in the $Z H \rightarrow \nu \bar{\nu} b \bar{b}$ channel continues with the continuous accumulation of luminosity by D $\varnothing$. As of February 2009, approximately $6 \mathrm{fb}^{-1}$ were delivered by the Tevatron and, from more than $5 \mathrm{fb}^{-1}$ recorded by the $\mathrm{D} \varnothing$ detector, $4 \mathrm{fb}^{-1}$ were processed with the $\mathrm{p} 20$ version of the offline software. This chapter covers work in progress.

Refinements in analysis techniques and experimental methods can help improve the sensitivity on top of the statistical improvement from additional data. Compared to the p17 analysis (Ch. 4), the cut on $E_{T}$ was lowered by $10 \mathrm{GeV}$, events with jets in the ICR $(1.1<|\eta|<1.4)$ were included, the treatment of events with bad jets was improved, the $\mathbb{E}_{T}$ significance algorithm was used to reject poorly measured events and an orthogonal single $b$-tag channel was added. The areas of improvement are described in more detail in the following sections. 


\begin{tabular}{rcccc} 
Trigger version & $\mathrm{v} 15-\mathrm{v} 15.20$ & $\mathrm{v} 15.20-\mathrm{v} 16$ & $\mathrm{v} 16-$ & Total \\
\hline Luminosity $\left(\mathrm{pb}^{-1}\right)$ & 209.5 & 1415.6 & 1411.1 & 3036 \\
\hline
\end{tabular}

Table 5.1: Integrated recorded luminosity $\left(\mathrm{pb}^{-1}\right)$ used in the analysis for each trigger version in Run IIb.

\subsection{Samples}

\subsubsection{Data}

All Run IIa data starting from trigger list v12 (Table 4.1) are used for the search. For data collected since spring 2006 (Run IIb), the trigger system was improved [66], allowing in particular the $\not_{T}$ to be used at the first level, and the $\not_{T}$ threshold to be lowered to $25 \mathrm{GeV}$. After data quality requirements are placed on Run IIb data, the integrated luminosity that we measure for each version of the trigger can be seen in Table 5.1.

\subsubsection{Monte Carlo Simulations}

The CTEQ6L1 LO parton distribution function (PDF) set $[67,68]$ was used to generate MC samples. All $W / Z+$ jets and $t \bar{t}$ processes were generated with ALPGEN v2.11 interfaced with PYTHIA v6.413 for the simulation of initial and final state radiation, and jet hadronisation. The generated partons in ALPGEN are required to have $p_{T}>8 \mathrm{GeV}$.

The ALPGEN cross sections used in the analysis are calculated at leading order (LO). Since next-to-leading order corrections are large, K-factors have to be applied to the leading-order cross sections. These are listed in Table 5.2. The light flavour K-factors were determined from the data before $b$-tagging. The heavy flavour fractions have been obtained using MCFM. The $Z p_{T}$ 


\begin{tabular}{ll} 
Sample & K-factor \\
\hline$W(\rightarrow l \nu)+$ light flavours & 1.3 \\
$W(\rightarrow l \nu)+\mathrm{cc}$ & $1.3 \times 1.47$ \\
$W(\rightarrow l \nu)+\mathrm{bb}$ & $1.3 \times 1.47$ \\
\hline$Z(\rightarrow l l, \rightarrow \nu \nu)+$ light flavours & 1.3 \\
$Z(\rightarrow l l, \rightarrow \nu \nu)+\mathrm{cc}$ & $1.3 \times 1.67$ \\
$Z(\rightarrow l l, \rightarrow \nu \nu)+\mathrm{bb}$ & $1.3 \times 1.52$ \\
\hline$t \bar{t}$ & 1.43 \\
$\operatorname{single} \mathrm{top} \rightarrow l \nu b$ & 0.99 \\
$\mathrm{WW}\left(\rightarrow l \nu l^{\prime} \nu\right)$ & 1.0 \\
$\mathrm{WZ}\left(\rightarrow l \nu l^{\prime} l^{\prime}\right)$ & 1.06 \\
$\mathrm{ZZ}\left(\rightarrow l l l^{\prime} l^{\prime}\right)$ & 1.03 \\
\hline$Z H \rightarrow \nu \bar{\nu} b b\left(m_{H}=115 \mathrm{GeV}\right)$ & 1.24 \\
$W H \rightarrow l \nu b \bar{b}\left(m_{H}=115 \mathrm{GeV}\right)$ & 1.21 \\
\hline
\end{tabular}

Table 5.2: $K$-factors used for background and signal Monte Carlo samples. spectrum was reweighted so as to match the $\mathrm{D} \varnothing$ measurement [69]. The $W$ $p_{T}$ spectrum was reweighted as well, based on the same input but taking into account the differences between the $Z$ and $W$ spectra predicted by a NNLO computation [70].

Diboson MC samples were produced with PYTHIA v6.413 and single top samples were produced with COMPHEP [71]. Top and diboson cross sections are taken from NNLO predictions $[72,73,74]$. Signal $(Z H \rightarrow \nu \bar{\nu} b \bar{b}$ and $\left.W H \rightarrow \ell \nu_{\ell} b \bar{b}\right)$ production was simulated using PYTHIA v6.413 and production cross sections were taken from [75].

Events were weighted to compensate for residual differences between data and simulation for luminosity profile, primary vertex longitudinal ( $z$-axis) distribution, and for electron, muon and jet identification. 


\subsection{Increased Signal Acceptance}

To increase the signal acceptance, the cut on the $E_{T}$ of the event was lowered from $50 \mathrm{GeV}$ to $40 \mathrm{GeV}$. Relaxing the $\not_{T}$ requirement increases the signal acceptance by $\sim 15 \%$. At the same time, the background acceptance is increased by a factor of 2 . The background composition for events with low $E_{T}$ is dominated by instrumental and mismeasured multijet events, as seen in Figs. 5.6, 5.6. Cutting on the $\not_{T}$ significance (see Sec. 5.4) can provide the necessary background rejection.

Jets reconstructed using the jet algorithm [76] with cone size $\Delta \mathcal{R}=0.5$ are required to pass a set of identification criteria [77]:

- $0.05<$ fraction of energy deposited in EM calorimeter layers (EM fraction $)<0.95$

- fraction of energy deposited in the $\mathrm{CH}$ calorimeter layer ( $\mathrm{CH}$ fraction) $<0.40$

- Ratio of energies in the cells with the highest and second highest energy deposits $<10$

- No more than $90 \%$ of the jet energy contained in one calorimeter cell

If a jet fails one or more of these criteria it is classified as bad. Events with jets classified as bad by the jet identification criteria produced a skewed $\mathcal{A}\left(E_{T}, H_{T}\right)$ distribution in the p17 analysis, as the energy deposits were taken into account for calculating the $E_{T}$ but not for the $\not_{T}$. Events with bad jets with $p_{T}>15 \mathrm{GeV}$ are now rejected as there is no expectation for such 
events in the MC simulation of the $Z H \rightarrow \nu \bar{\nu} b \bar{b}$ signal. There is however a significant proportion $(\sim 30 \%)$ of the electrons from $W H$ production where the $W$ decays to an electron that fail the electron identification criteria and are identified as jets. Most of these jets are classified as bad due to high EM fraction and appear as a third jet along the two $b$-jets from the Higgs decay. To reclaim the signal lost in this way, events with three jets are not rejected if they have only one bad jet with $p_{T}>15 \mathrm{GeV}$, if the only criterion for the jet to be classified as bad is the fraction of the jet energy deposited in the EM calorimeter.

After analysis cuts are placed, around $20 \%$ of the $V H$ signal events have at least one of the two leading jets in $p_{T}$ fall within the ICR $(1.1<|\eta|<1.4)$. These events were rejected in Run IIa data because of hot regions in the ICR and the very high instrumental background rate. During the Tevatron shutdown between Run IIa and Run IIb, the fine hadronic calorimeter was re-calibrated [78]. The quality of the calibration of the scintillating tiles of the inter-cryostat detector was greatly improved allowing for the inclusion of events with jets in the ICR in the analysis. The use of the full $\eta$ acceptance of the $\mathrm{D} \varnothing$ calorimeter for the majority of the data collected greatly enhances the signal acceptance from previous analyses.

In addition, the cut on the $p_{T}$ of the next-to-leading jet could in the future be lowered to $15 \mathrm{GeV}$ to increase signal acceptance. Approximately $60 \mathrm{pb}^{-1}$ were collected using a missing energy and acoplanar jet trigger in trigger version 11 . These data can potentially be added but additional effort would be required to parametrise the Level-3 requirements (the jet cone size used is different to that in subsequent versions of the trigger). Additional 
acceptance at the trigger level could potentially be achieved by combining the trigger requirements presently used with complementary ones such as the triggers requiring the identification of $b$-jets.

In total, relaxing the basic kinematic cuts and adding more advanced selection requirements allow for an absolute signal efficiency close to $90 \%$ after the trigger requirements. Multivariate techniques for event classification and $b$-tagging can then be used to enhance the signal to background ratio.

\subsection{Multijet Background Treatment}

The background contribution from multijet processes and instrumental effects is modeled from data. As with previous versions of the analysis, we use the $\Delta \phi\left(\mathbb{Z}_{T}, E_{T}\right)>\pi / 2$ cut (see Sec. 4.4.2 for definitions) to define a sideband region with no signal expectation that allows us to model background events where the jet energies are mismeasured, leading to significant $\mathbb{E}_{T}$ aligned with the jets (Fig. 5.1).

Previous versions of the analysis computed $\mathbb{T}_{T}$ from tracks identified with at least 8 hits in the CFT layers. However, the requirement of a high number of CFT hits can cause tracks not to be counted and associated with a jet in signal events and thus cause these events to be classified as instrumental by shifting the direction of the $\mathbb{T}_{T}$. This is likely to happen more often in signal events that have forward jets with tracks that do not traverse all the CFT layers. This requirement can thus impose a bias in the estimate of the instrumental background and was dropped.

To suppress the background contribution from instrumental effects fur- 


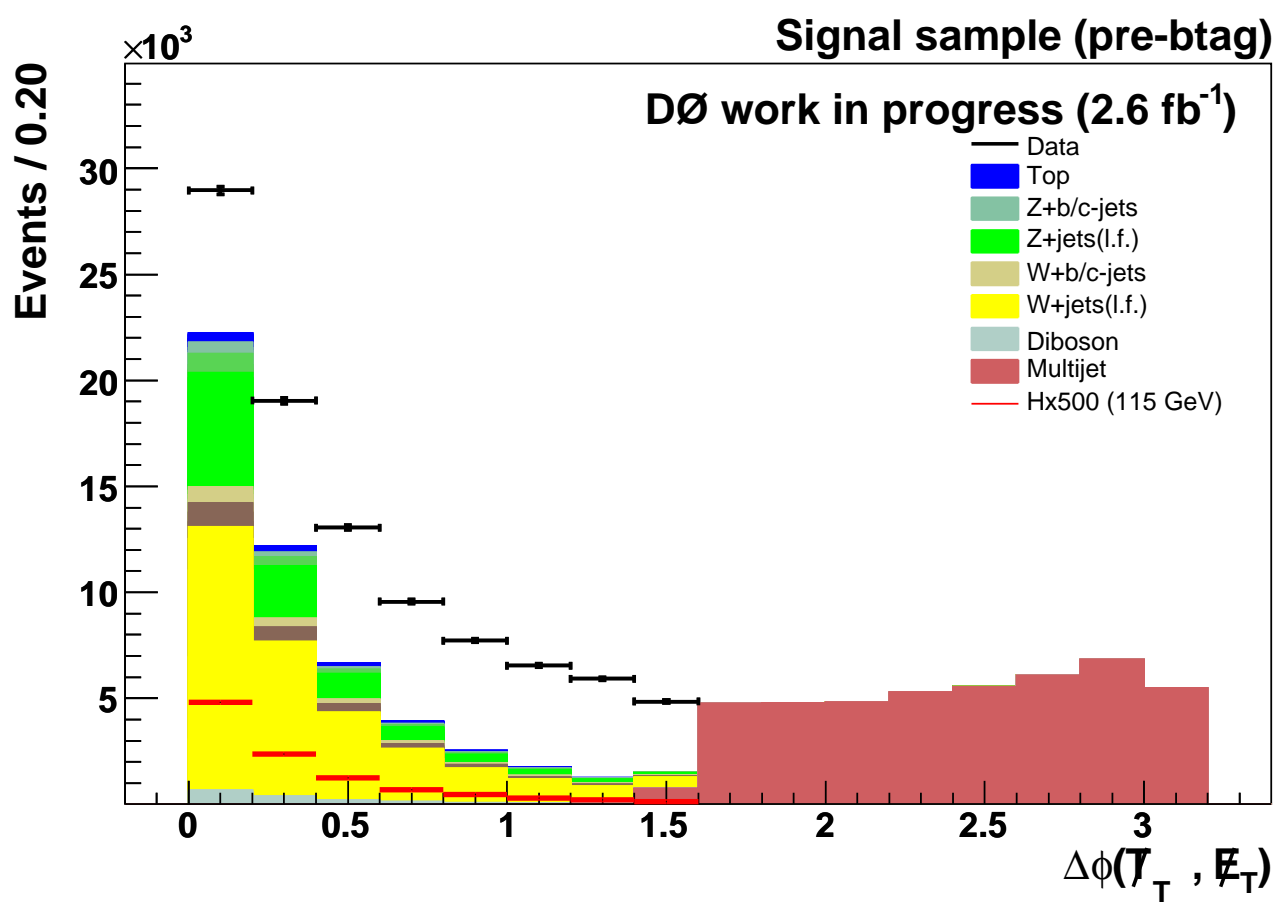

Figure 5.1: $\Delta \phi\left(\mathbb{F}_{T}, \mathbb{E}_{T}\right)$ for Run IIb data with integrated luminosity $2.6 \mathrm{fb}^{-1}$ before $b$-tagging is applied. The background expectation from physical processes modeled by $\mathrm{MC}$ and the signal expectation are shown in the region $\Delta \phi\left(\mathbb{T}_{T}, \mathbb{E}_{T}\right)<\pi / 2$. The difference between the MC expectation and the data observed corresponds to the multijet background contribution which is modeled by the data in the sideband region after subtracting the MC expectation shown in the region $\Delta \phi\left(\mathbb{Z}_{T}, \mathbb{E}_{T}\right)>\pi / 2$. 
ther, a cut can be placed on the two-dimensional distribution of jet $p_{T}$ measured with the calorimeter and the tracking (the vectorial $p_{T}$ sum of all tracks in the jet).

To test our modeling of multijet and instrumental events, we define a control sample (referred to as the multijet control sample onwards) where effects of this nature are enhanced. This sample is identical to the analysis sample, but with the cut on $E_{T}$ relaxed from 40 to $30 \mathrm{GeV}$ and all the cuts that reject multijet events dropped. As a result, multijet events largely dominate not only the sideband region, but also the signal region. This sample is then used to verify how well the events with $\Delta \phi\left(\mathbb{F}_{T}, \mathbb{E}_{T}\right)>\pi / 2$ provide a model for those with $\Delta \phi\left(\mathbb{T}_{T}, \mathbb{E}_{T}\right)<\pi / 2$.

The expected number of events in the multijet control sample with $\Delta \phi\left(\mathbb{P}_{T}, \not_{T}\right)<$ $\pi / 2$ is normalised to the number of events observed. Separate normalisation factors are obtained for Run IIa and Run IIb, due to the differences in the trigger requirements and the inclusion of the ICR. As can be seen in Figs. 5.2, 5.3, calorimetric variables are very well described by the multijet model.

There are some differences in distributions related to the tracking which can have an effect on the $b$-tagging algorithms. Also, semi-leptonic heavy flavour decays in the multijet sample produce events with intrinsic $E_{T}$ aligned with the jets. For these reasons, the selection efficiency is expected to be different when requirements are placed using the NN b-tagger. The distributions of the $b$-tagging $\mathrm{NN}$ output for the jets in the multijet control sample are shown in Fig. 5.4 and disagreement between observation and expectation is visible for high values of the NN output. To take this effect into account, 

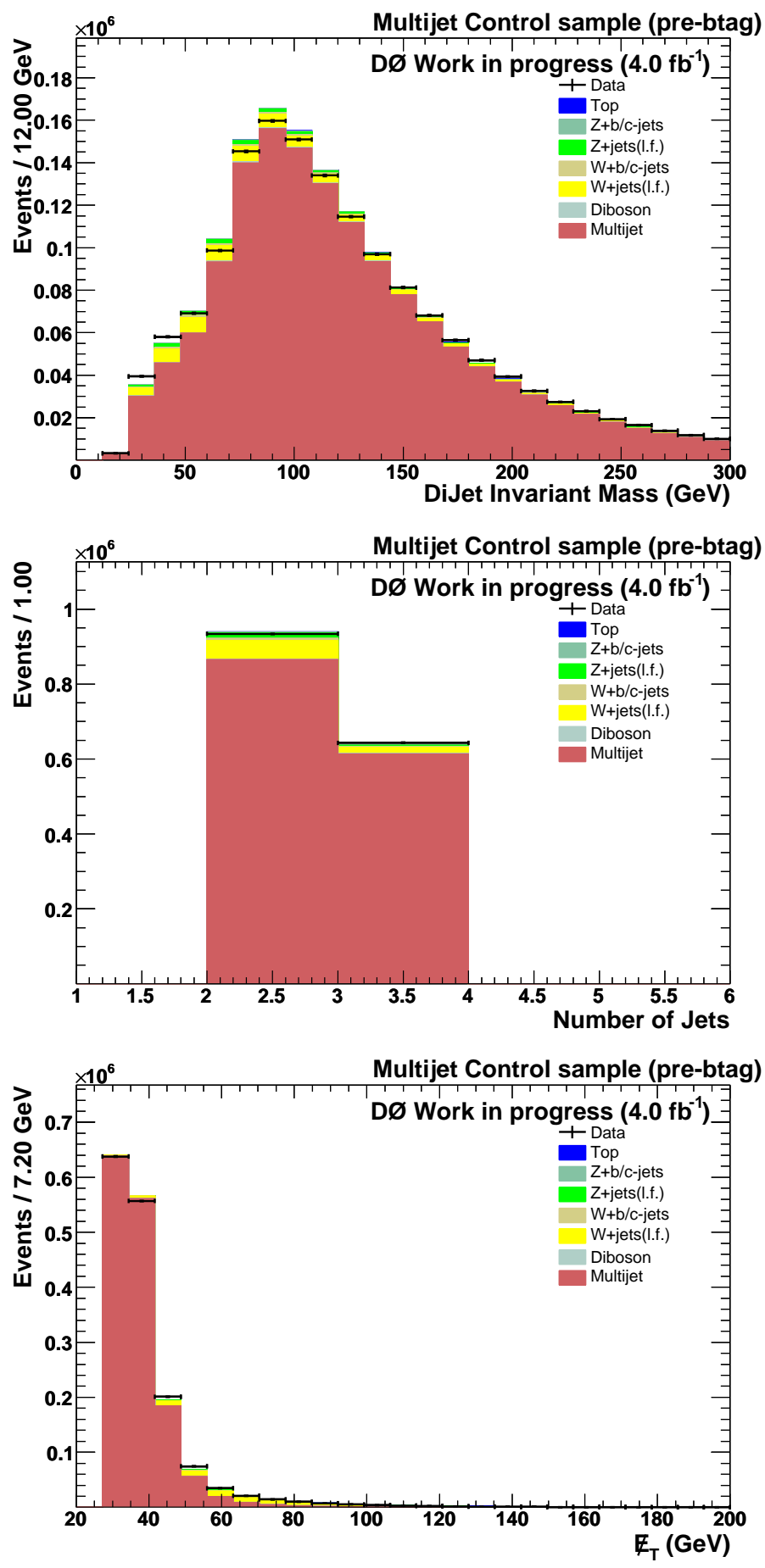

Figure 5.2: Multijet control sample before b-tagging. 

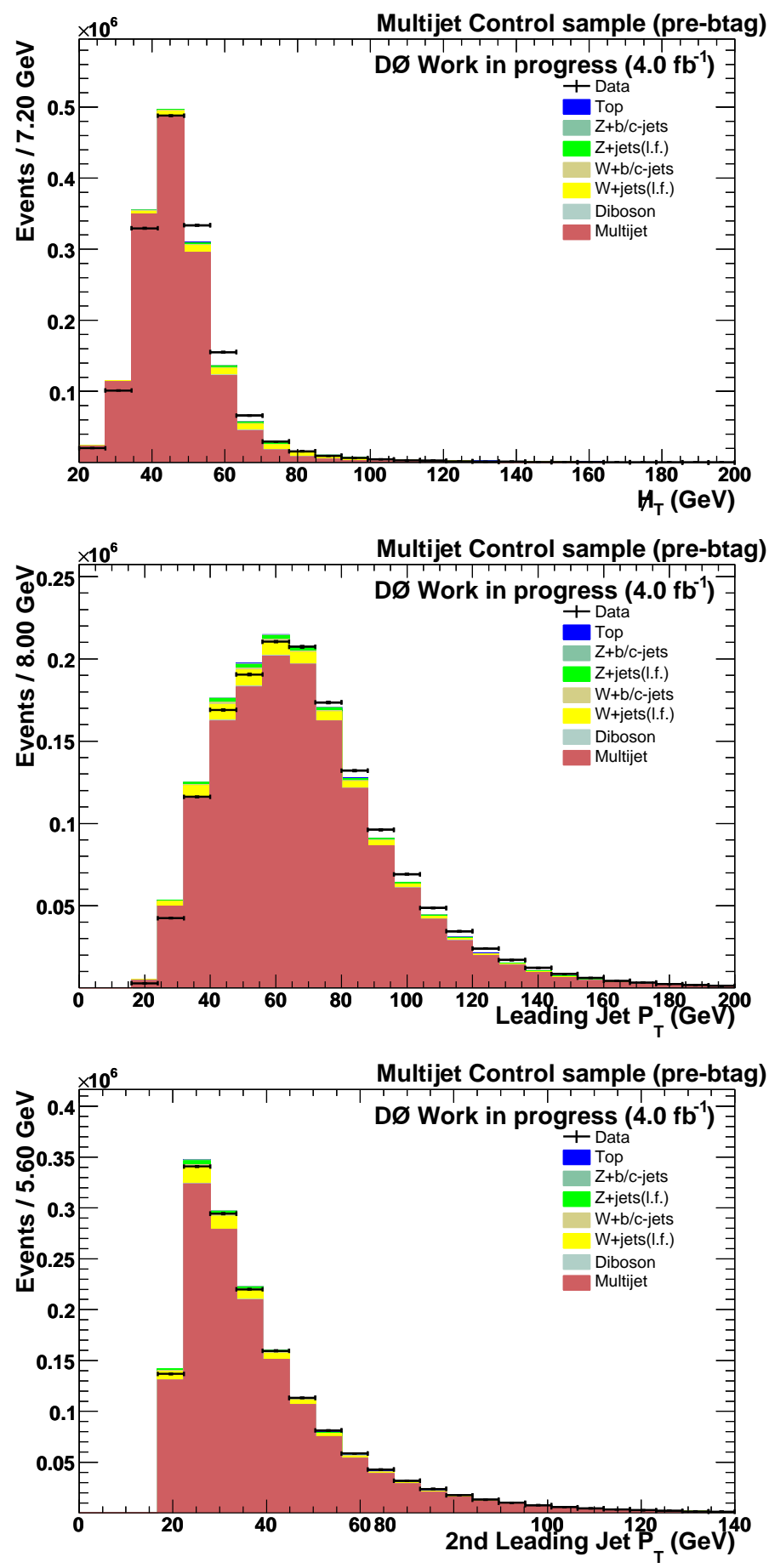

Figure 5.3: Multijet control sample before b-tagging. 

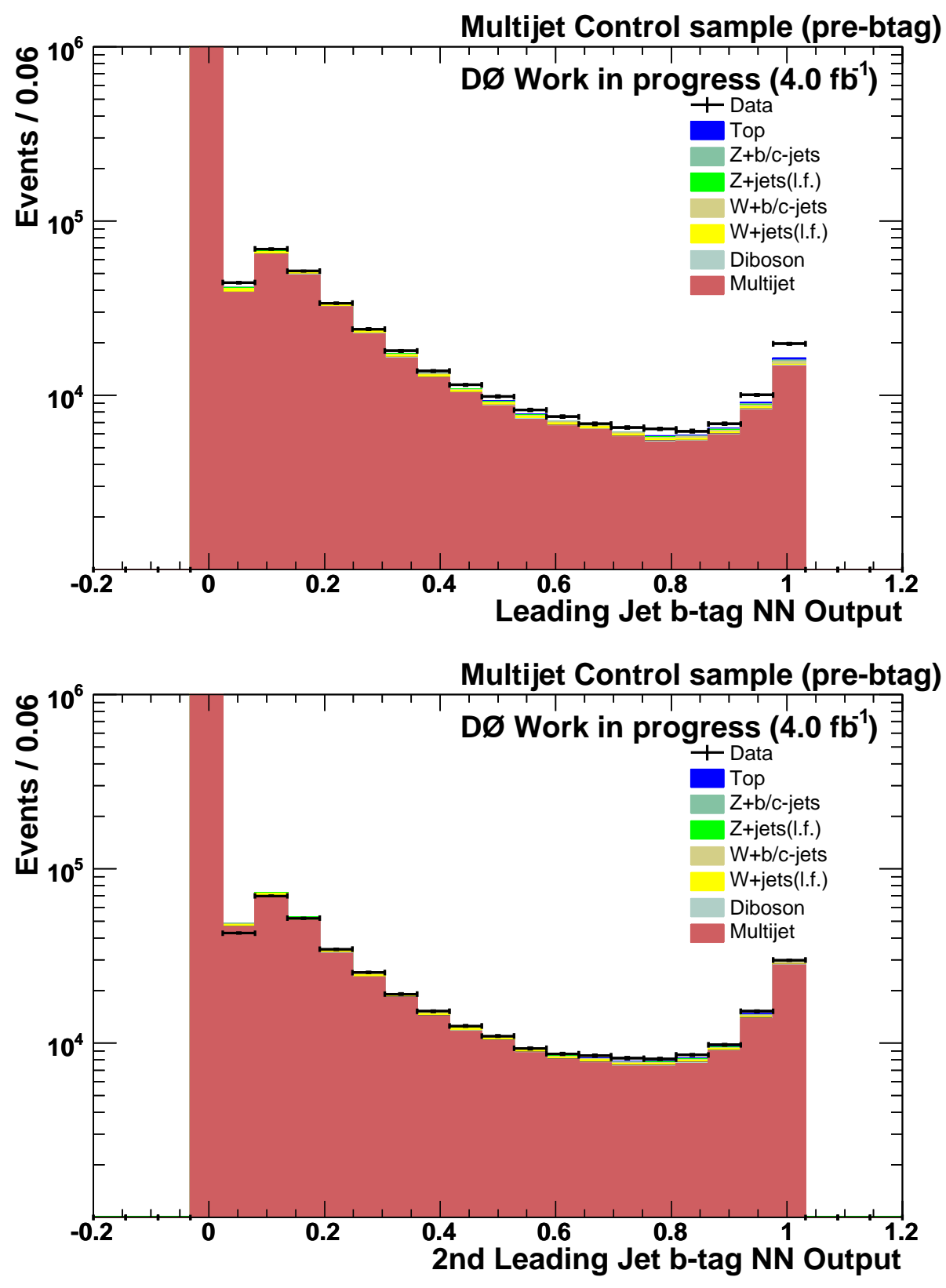

Figure 5.4: Leading and next-to-leading jet $b$-tag NN output distributions in the multijet dominated sample. 
a different normalisation factor is used for the pre-tagged and each b-tagged multijet sample. The ratio of the $b$-tagged and the pre-tagged scale factors in the mutlijet control sample is used to normalise the multijet contribution at the equivalent $b$-tag point in the signal sample.

\subsection{The $\mathbb{E}_{T}$ Significance}

A probabilistic method can be implemented to evaluate the significance of the measured $\mathbb{E}_{T}$ in each event based on its resolution. The $E_{T}$ significance method was originally developed during Run I of the Tevatron [79] and the underlying algorithm [80] was adapted and optimised for Run II [81]. The $\mathbb{E}_{T}$ significance, $\mathcal{S}$, can be used to discriminate events with true missing energy from events which are poorly or erroneously measured.

The missing transverse energy resolution of an event is determined by a multitude of effects. The most prominent are the identification of the vertex location, the energy resolution of jets, electrons and muons and calorimetric effects such as unclustered energy deposits and the appearance of hot cells. Based on the knowledge of energy resolutions of the physics objects in an event, a probability distribution is computed for the $\mathbb{E}_{T}$, that evaluates how likely it is that the measured $E_{T}$ is consistent with a resolution fluctuation of the measured objects in its direction.

For the purposes of the computation, it is assumed that the probability densities of the energy of individual physics objects are Gaussian distributions. Three different physics objects are considered:

Jets The jet energy resolution is obtained from the $p_{T}$ momentum imbalance 
in back-to-back dijet events, parametrised as a function of jet $E_{T}$ in four $\eta$ regions. The jet resolutions were updated to the latest ones measured for Run IIb for the purposes of this analysis.

Unclustered Energy Unclustered energy results from the presence of soft jets, out-of-cone energy and warm and noisy regions.

Electrons and Photons The resolution of electromagnetic objects is derived from Monte Carlo simulations.

The distribution of the $E_{T}$ significance variable in the multijet control sample is shown in Fig. 5.5. The instrumental background that dominates the sample composition is concentrated and peaks at low values of $E_{T}$ significance. The physical processes that are simulated by MC, which have 


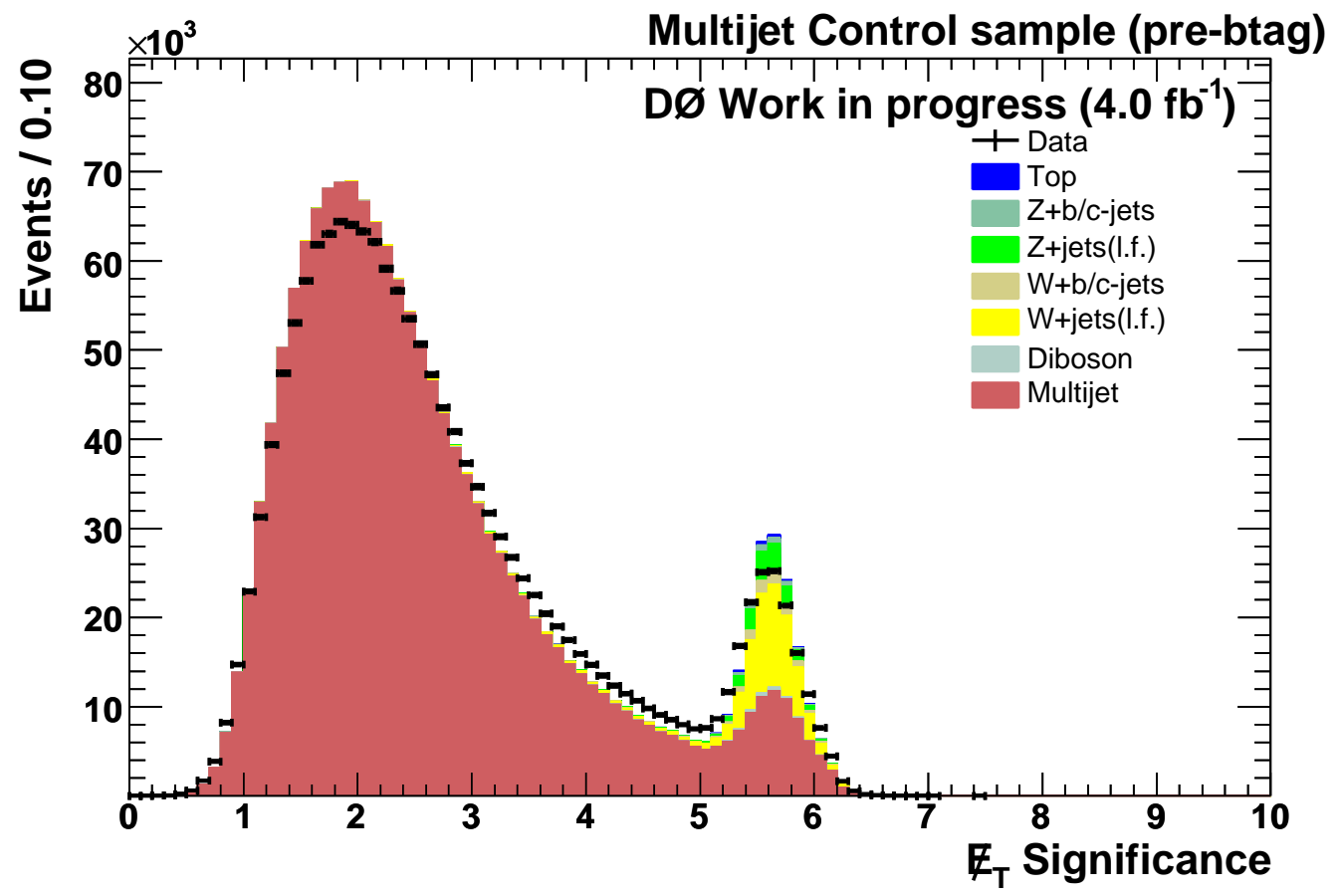

Figure 5.5: Distribution of the $E_{T}$ significance variable in the multijet control sample. The apparent disagreement between the expected and observed distributions is related to the limited modeling of instrumental and multijet effects, especially for events with $E_{T}<40 \mathrm{GeV}$, before event selection cuts are placed. 
Events with isolated electrons or muons with $p_{T}>15 \mathrm{GeV}$ are removed. The efficiency of lepton identification in $\mathrm{MC}$ is corrected to match that measured in data. The electron and muon definitions are adapted to be orthogonal to other $\mathrm{D} \varnothing$ searches.

The final event selection as described in the text is listed for clarity:

- Two or three jets with $p_{T}>20 \mathrm{GeV}$ and $|\eta|<2.5$.

- $\left|z_{P V}\right|<40 \mathrm{~cm}$, and at least 3 tracks attached to the PV.

- $E_{T}>40 \mathrm{GeV}$.

- No bad jets in the event with $p_{T}>15 \mathrm{GeV}$, except jets with the only bad criterion being the jet EM fraction $>0.95$.

- No isolated electrons or muons in the event (statistically independent to other DØ channels)

In addition, topological cuts are applied to reduce the multijet/instrumental backgrounds:

- Acoplanarity $\Delta \phi\left(\right.$ jet $_{1}$, jet $\left._{2}\right)<165^{\circ}$

- $E_{T}(\mathrm{GeV})>-40 \times \min \Delta \phi\left(E_{T}\right.$, jets $)+80$

- $\Delta \phi\left(\mathbb{E}_{T}, \mathbb{T}_{T}\right)<\pi / 2$, where $\mathbb{T}_{T}$ is the missing $p_{T}$ from tracks

- $-0.1<\mathcal{A}\left(E_{T}, \not_{T}\right)<0.2$, where $\mathcal{A}\left(E_{T}, \not_{T}\right) \equiv\left(E_{T}-H_{T}\right) /\left(E_{T}+\not T_{T}\right)$

- $E_{T}$ Significance $\mathcal{S}>5$ 


\subsection{Taggability and $b$-tagging}

To take advantage of the large branching fraction for $H \rightarrow b \bar{b}$ we require that at least two jets in each event are vertex-confirmed (at least two tracks associated with the PV) and taggable and that one or both of the two leading taggable jets be $b$-tagged. The vertex-confirmation requirement removes a large fraction of the instrumental background by rejecting events with fake jets, or events where the primary vertex was misidentified. Asymmetric (tight-loose) cuts are placed on the outputs of the standard D $\varnothing b$-tagging algorithm neural network which were found to provide the best sensitivity to a Higgs boson signal (Sec. 4.7.3). To enhance the search sensitivity by increasing signal acceptance, an orthogonal sample is also considered, with one tightly tagged jet and no other jet loosely tagged in every event.

Agreement between data and expectation from SM and multijet backgrounds is observed in terms of numbers of events selected (Table 5.3). The sensitivity of the search in terms of the signal to background ratio has been enhanced significantly when compared to previous versions of the analysis. Variable distributions before $b$-tagging can be seen in Figs. 5.6, 5.7, for a sample with one $b$-tagged jet that is orthogonal to the double tag sample in Figs. 5.8, 5.9, and after double asymmetric tagging in Figs. 5.10, 5.11. Overall, there is good agreement between the expected background and the observed data, both before and after $b$-tagging. 

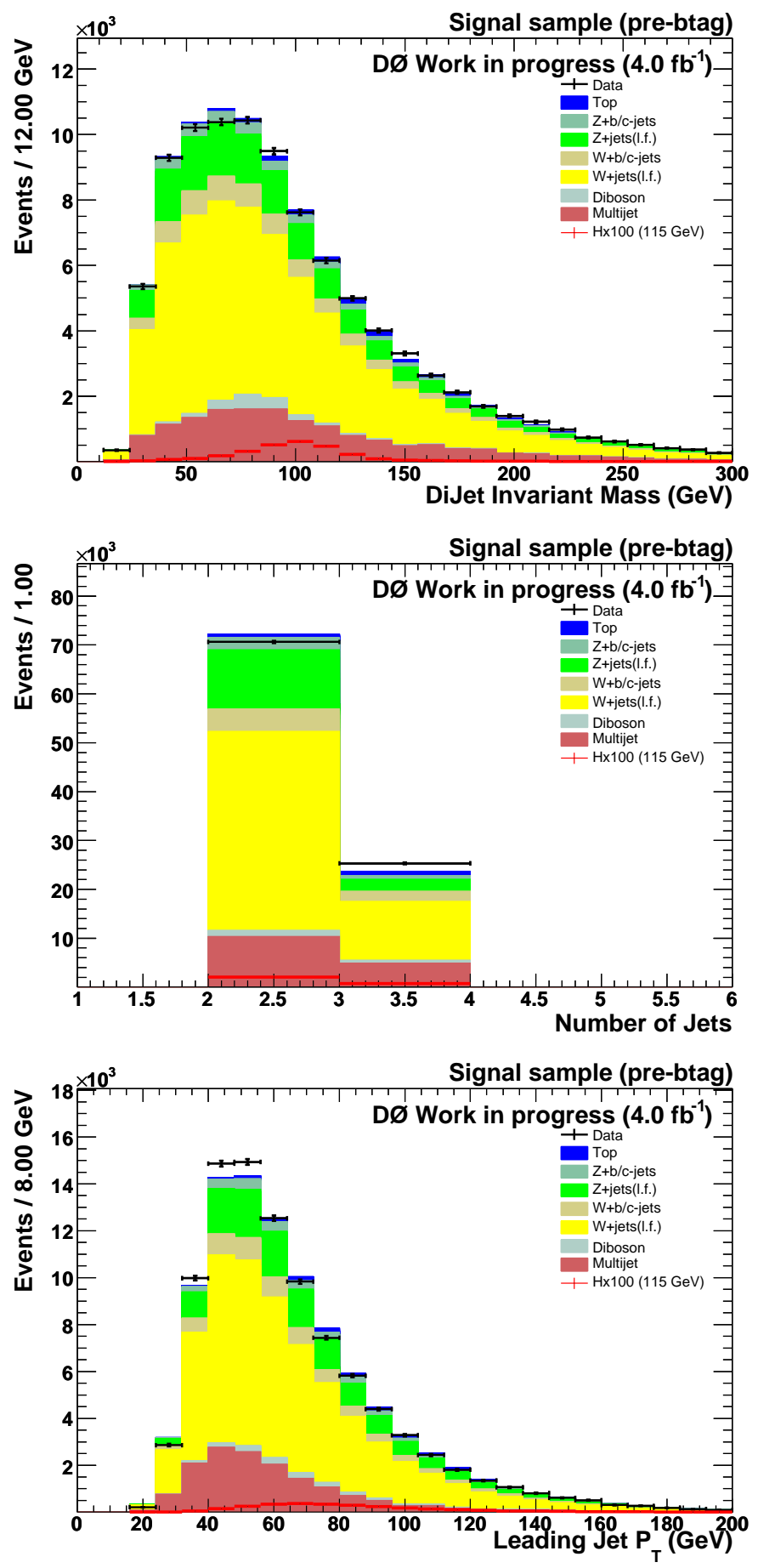

Figure 5.6: Signal sample before $b$-tagging 

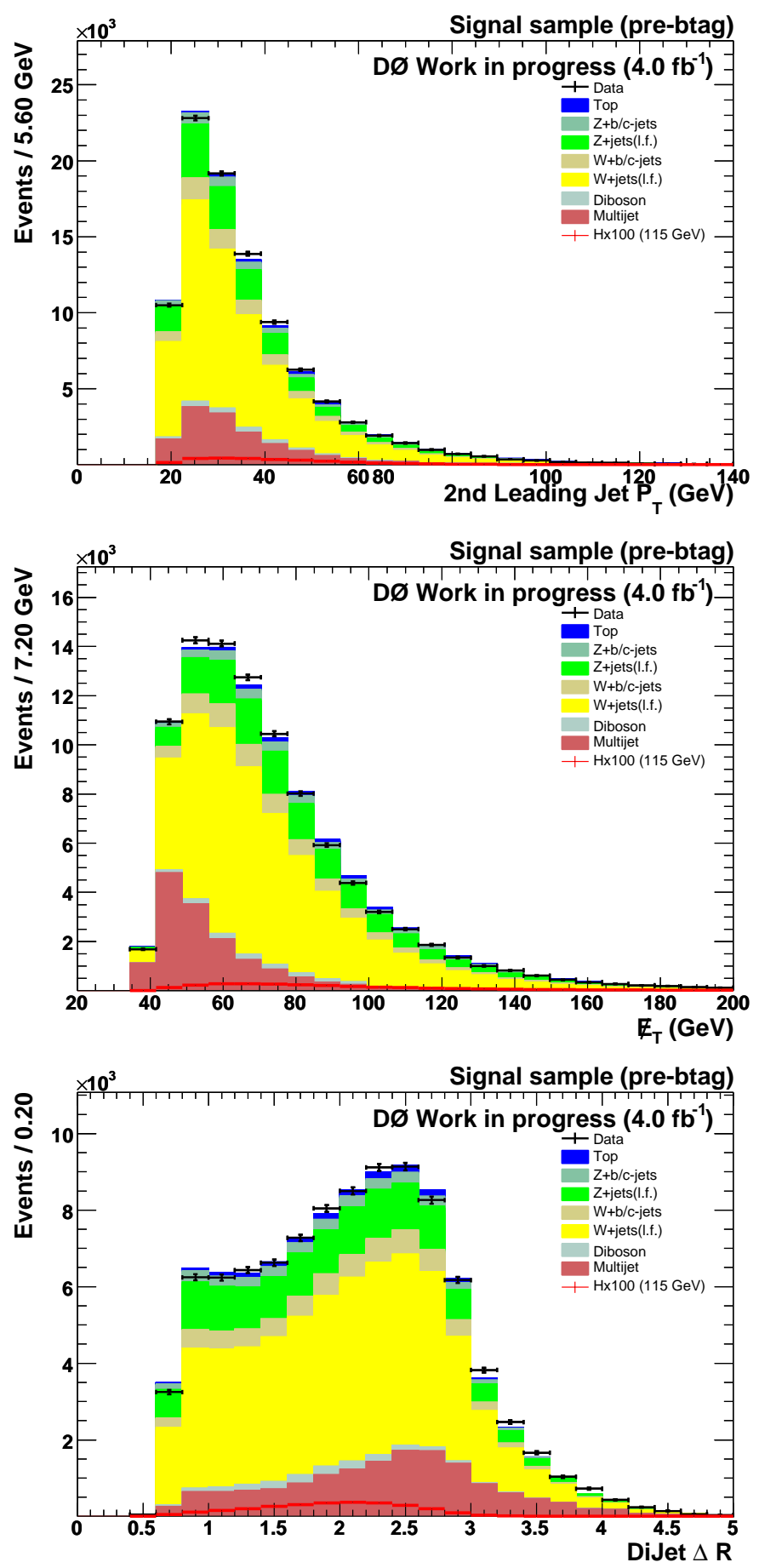

Figure 5.7: Signal sample before $b$-tagging 

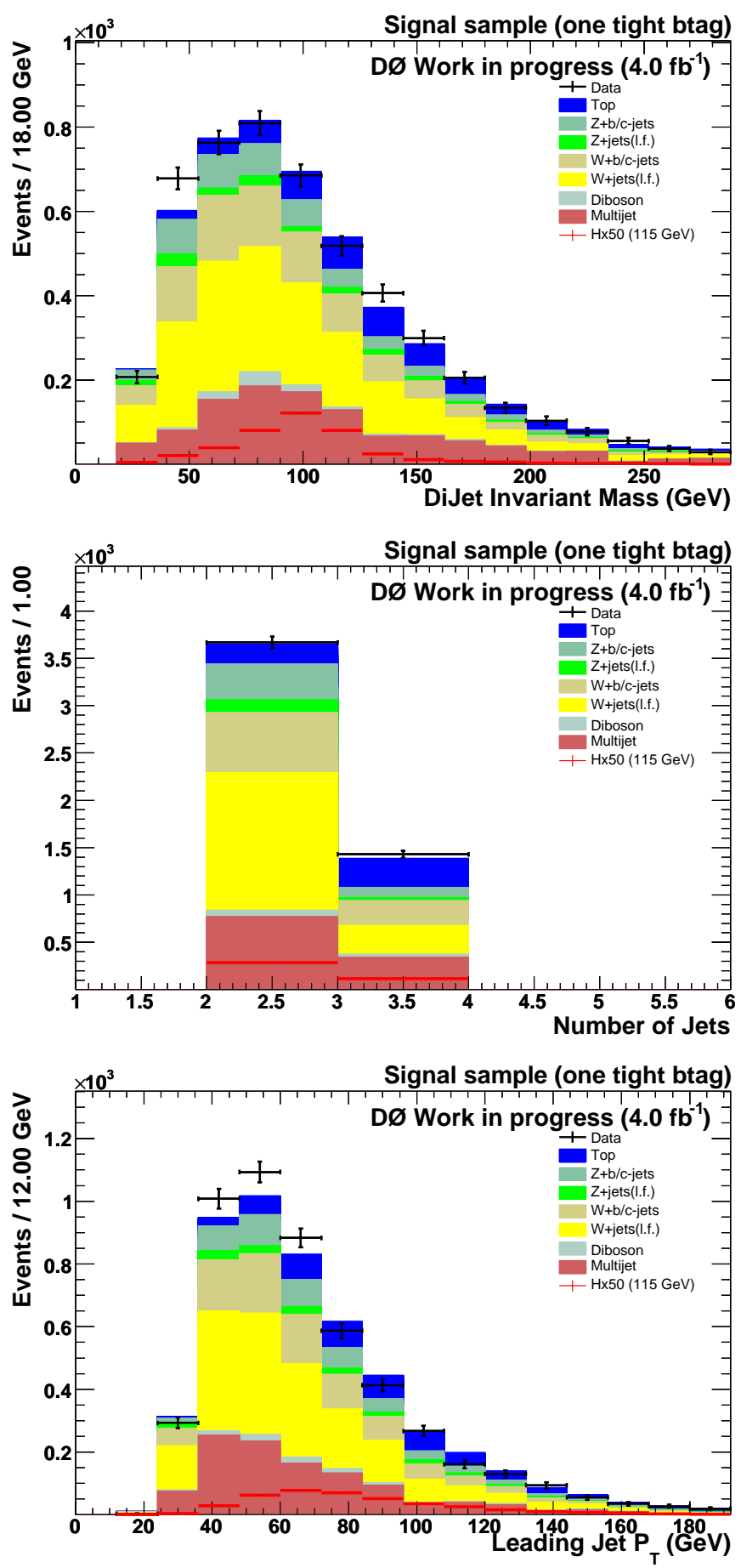

Figure 5.8: Signal sample with one tight $b$-tag 

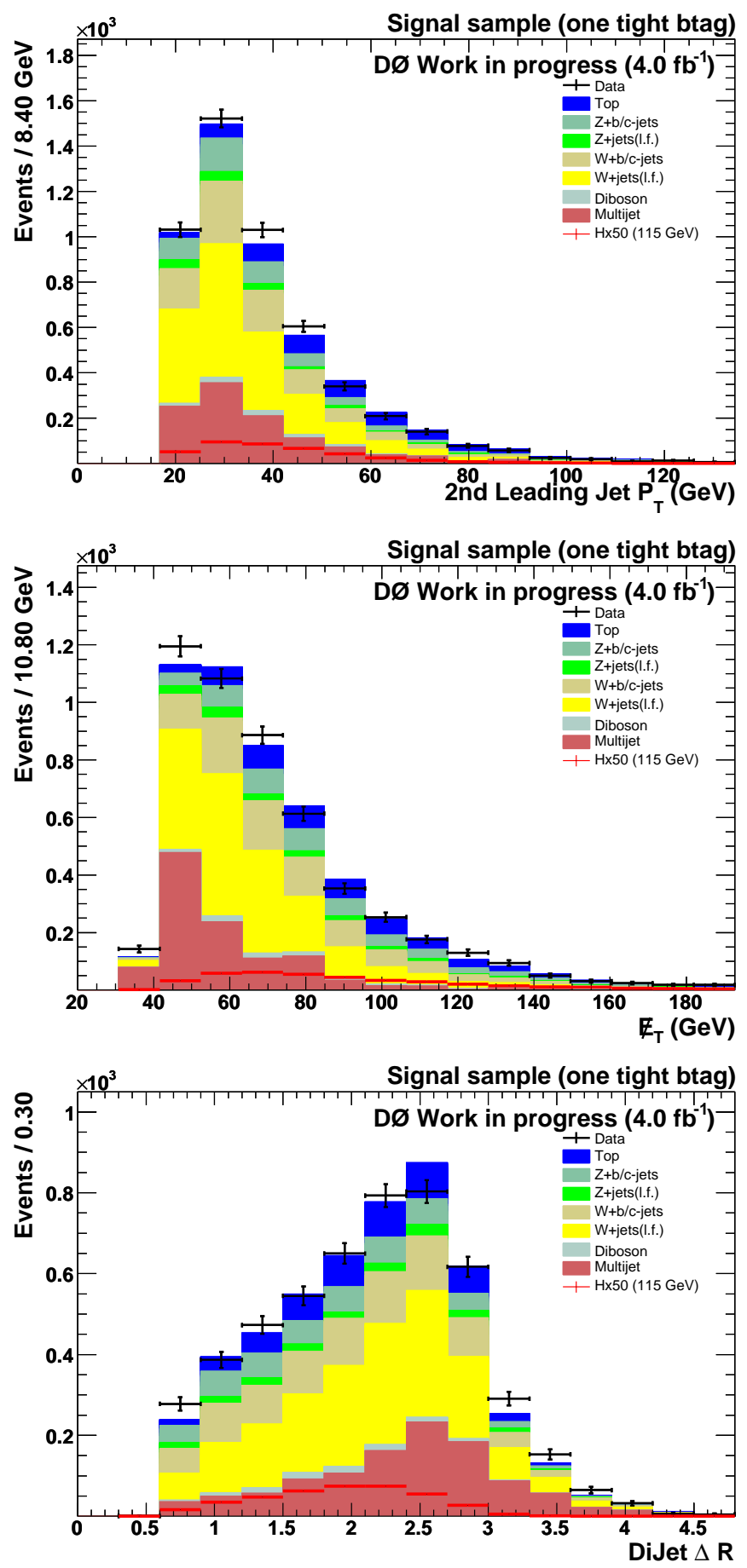

Figure 5.9: Signal sample with one tight $b$-tag 

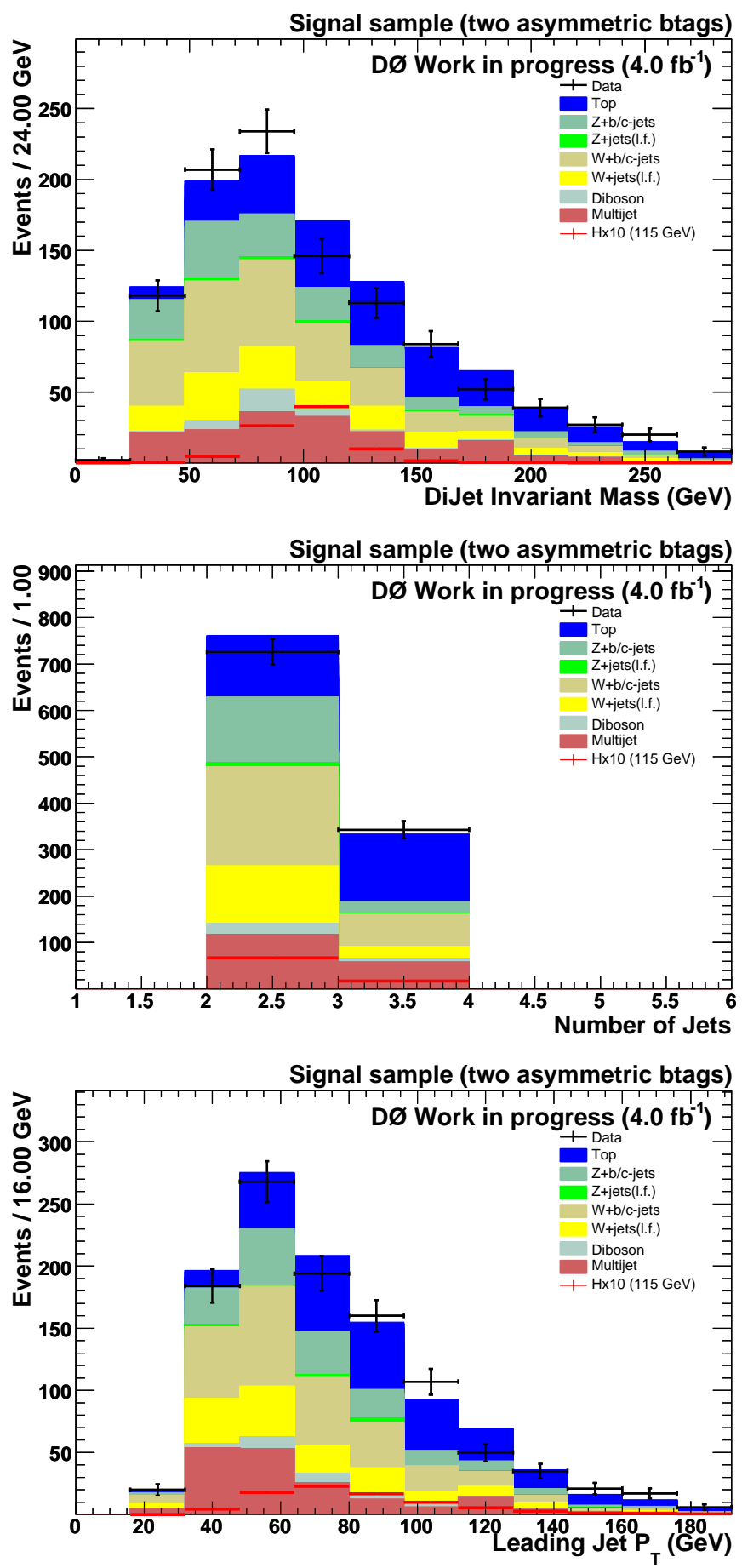

Figure 5.10: Signal sample with one tight and one loose $b$-tag 

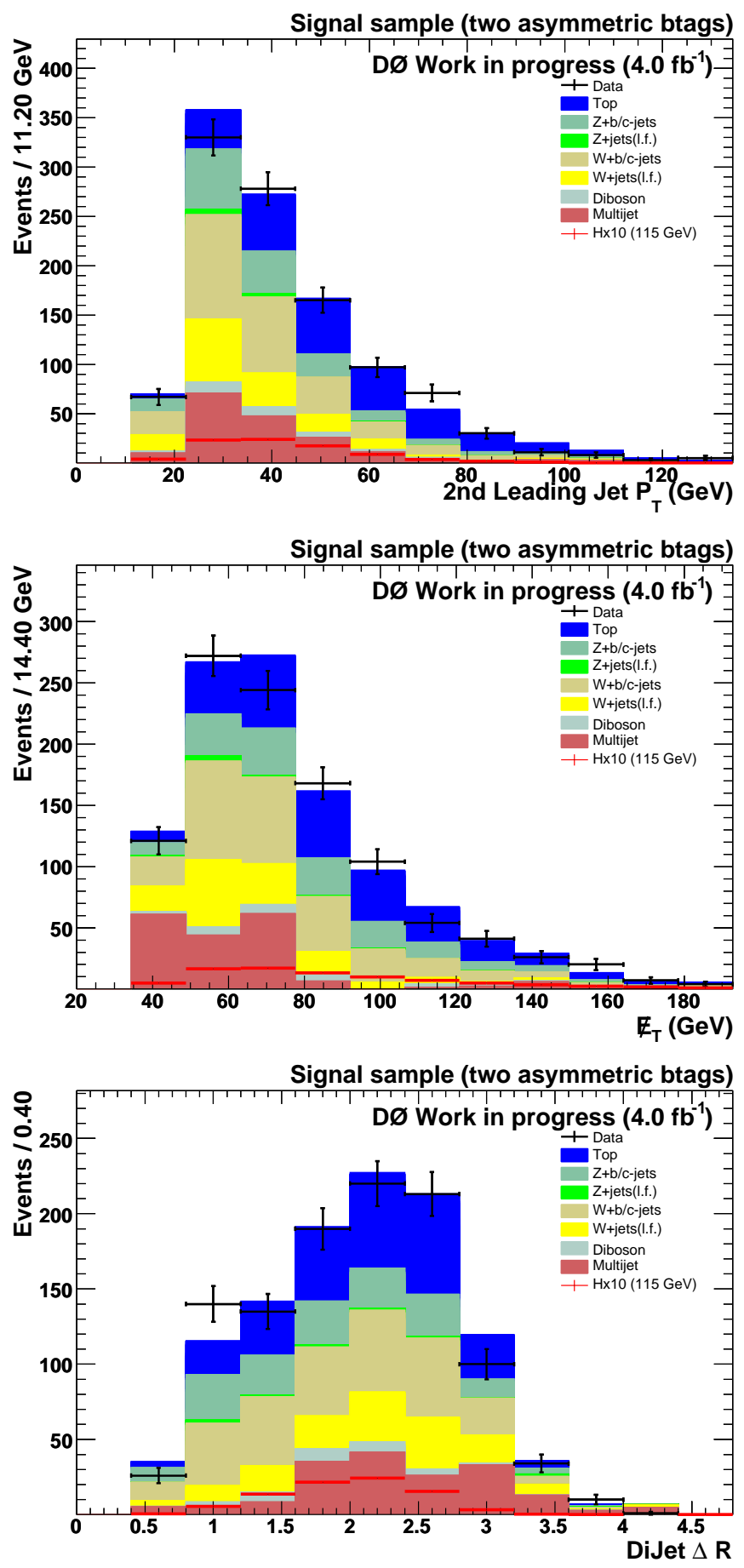

Figure 5.11: Signal sample with one tight and one loose $b$-tag 


\begin{tabular}{rccc} 
Sample & Pre-tag & $1 b$-tag & 2 b-tags \\
\hline$Z H$ & $13.41 \pm 0.05$ & $3.83 \pm 0.01$ & $4.10 \pm 0.01$ \\
$W H$ & $13.62 \pm 0.07$ & $3.96 \pm 0.02$ & $4.08 \pm 0.02$ \\
\hline$W+$ jets & 59402 & 2654 & 433.0 \\
$Z+$ jets & 17866 & 654 & 177.0 \\
Top & 1607 & 517 & 278.0 \\
Diboson & 1837 & 99 & 31.1 \\
Multijet & 15238 & 1111 & 175.9 \\
\hline Total & & $5035 \pm 7$ & $1095 \pm 2$ \\
Observed & 95950 & 5099 & 1069 \\
\hline
\end{tabular}

Table 5.3: Expected numbers of signal and background events, and numbers of events observed before $b$-tagging, with one exclusive tight $b$-tag, and with asymmetric double $b$-tag. The numbers for the signals are given for a Higgs boson mass of $115 \mathrm{GeV}$; "Top" includes pair and single top production; "Diboson" stands for the sum of $W W, Z Z, W Z$ processes. The errors quoted are statistical only and exclude the error on the multijet background. The total background expectation is normalised to data at the pretag level.

\subsection{Systematic Uncertainties}

Systematic uncertainties originate from various sources. As with the p17 result (Sec. 4.10), experimental uncertainties on the numbers of predicted signal and SM background events arise from the trigger simulation, from the jet energy calibration, resolution, and reconstruction efficiency, and from $b$ tagging. The corresponding parametrisations applied to the simulation are varied within their uncertainties, and the impact on both the normalisations and the shapes of the final discriminants are assessed. The correlations between these systematic uncertainties in the signal and background processes are taken into account to derive the final results, as well as for the uncertainty on the integrated luminosity of the data sample.

The cross sections for the various SM processes suffer from theoretical uncertainties. For the $W / Z$ +jets process, the overall normalisation can be 
constrained by the data in the $W \rightarrow \mu \nu+$ jets control sample before $b$-tagging. For the other background processes, theoretical uncertainties are taken from Refs. $[72,73,74]$, or calculated with MCFM. They range from $6 \%$ to $10 \%$. The uncertainties on the signal acceptance due to the PDF choice are now estimated using the forty CTEQ 6.1M PDF error sets [67, 68], and propagated to the final discriminants.

\subsection{Outlook}

The dataset for the search for the Standard Model Higgs boson has been extended to include a total of $4.0 \mathrm{fb}^{-1}$ of $p \bar{p}$ collisions at $1.96 \mathrm{TeV}$ recorded during Run IIa and the beginning of Run IIb at the Tevatron. The topology analysed consists of a pair of acoplanar $b$ jets and large $\not_{T}$, as expected from the process $p \bar{p} \rightarrow Z H \rightarrow \nu \bar{\nu} b \bar{b}$. The search is also sensitive to $W H$ associated production, where the $W$ boson decays leptonically and the charged lepton is undetected.

These improvements, along with those under development, combined with the additional data, will significantly enhance the sensitivity of the search for the low mass SM Higgs boson in this channel as well as for the combined D $\varnothing$ and Tevatron searches. A new public result is expected this summer. 


\section{Chapter 6}

\section{Conclusion}

Significant work was carried out on the highest level trigger, Level-3 and the search for the SM low mass Higgs boson in the $Z H \rightarrow \nu \bar{\nu} b \bar{b}$ channel at D $\varnothing$.

The Level-3 $b$-tagging algorithm uses a probability distribution, parametrised from lifetime information in data, to produce an overall probability that the event originated from heavy or light quark production. By parametrising the lifetime probability in data, a $b$-tagging efficiency greater than $90 \%$ was achieved for a factor of two rejection over a wide $\left(\sim 1-3 \times 10^{32} \mathrm{~cm}^{-2} \mathrm{~s}^{-1}\right)$ range of instantaneous luminosities. This parameterisation was used to take data from the start of RunIIb of the Tevatron and is expected to be used throughout the lifetime of the $\mathrm{D} \varnothing$ experiment. Triggers utilising the L3 IP $b$-tagging tool, with a $b$-tag probability cut at 0.4 , are used in the search for the neutral Higgs boson in multi-b-jet events [82]. As the final state signal selection requires the presence of more than 2 high $p_{T} b$-jets, the online trigger $b$-tagging efficiency is close to $100 \%$, while the accepted background is reduced by a factor of two. Similar triggers can be used in the search for 
$Z H \rightarrow \nu \bar{\nu} b \bar{b}$, when the thresholds of topological triggers have to be raised to cope with high instantaneous luminosities.

We have performed a search for the standard model Higgs produced in association with either a $Z$ or $W$ boson (denoted as $V H$ ), in the final state topology requiring missing transverse momentum and two $b$-tagged jets in $0.93 \mathrm{fb}^{-1}$ of data. There are two types of backgrounds: physical processes modeled by Monte Carlo (MC) and instrumental background predicted from data. Artificial neural networks (NN) were used for jet $b$-tagging and to classify events based on topological information from kinematic variables. The use of the NN output as the discriminating variable gives a $20 \%$ improvement on the expected limit with respect to the use of the dijet invariant mass. Systematic uncertainties affect the expected number of signal and background events as well as the shape of the distribution of the NN output. The inclusion of the systematic uncertainties causes a $\sim 25 \%$ degradation of the expected limit.

In the absence of a significant excess in data above the background expectation, we set limits on $\sigma(p \bar{p} \rightarrow V H) \times B(H \rightarrow b \bar{b})$ at the $95 \%$ confidence level of $2.6 \mathrm{pb}-2.3 \mathrm{pb}$ for Higgs boson masses in the range $105-135 \mathrm{GeV}$ shown in Table 4.7. At the time of publication [1], this was the most stringent limit using the missing energy and acoplanar $b$-jet topology at a hadron collider. The result was combined with the results of searches in complementary decay modes by $\mathrm{D} \varnothing[51,52]$ and together with CDF [83] by the Tevatron New Phenomena \& Higgs Working Group.

Refinements in analysis techniques and experimental methods can help improve the sensitivity on top of the statistical improvement from additional 
data. The treatment of the multijet background was refined and a control region rich in instrumental and multijet content was implemented to ascertain the validity of our background modeling procedure. Through the improved background modelling it was possible to relax the cut on $\mathbb{E}_{T}$, resulting in $\sim 30 \%$ greater signal acceptance in the $2 b$-tag channel. All together the $S / \sqrt{B}$ was increased by a factor 2 in this channel. Furthermore, an additional increase in sensitivity has been achieved by introducing an orthogonal single $b$-tag channel in the search. A new result is to be published with these improvements and a significantly larger dataset. 


\section{References}

[1] The D0 Collaboration: V.M. Abazov et al. Search for the Standard Model Higgs boson in the Missing Energy and Acoplanar b-Jet Topology at $\sqrt{s}=1.96 \mathrm{TeV}$. Phys. Rev. Lett., (101):251802, 2008.

[2] The D0 Collaboration. A Search for the Standard Model Higgs Boson in the Channel $Z H \rightarrow \nu \bar{\nu} b \bar{b}$ at $\sqrt{s}=1.96 \mathrm{TeV}$. D0 Note 5353-CONF, April 2007.

[3] The D0 Collaboration. A Search for the Standard Model Higgs Boson in the Channel $Z H \rightarrow \nu \bar{\nu} b \bar{b}$ at $\sqrt{s}=1.96 \mathrm{TeV}$. D0 Note 5506-CONF, November 2007.

[4] F. Halzen and A. Martin. Quarks \& Leptons. John Wiley \& Sons, 1984.

[5] D. Griffiths. Introduction to Elementary Particles. John Wiley \& Sons, 1987.

[6] Y. Nir. The Physics of Heavy Flavors. Lectures given at the '2007 CERN-FERMILAB Hadron Collider Physics Summer School', June 2007.

[7] G. Bernardi et al. Higgs Bosons: Theory and Searches. Particle Data Group, November 2007.

[8] K. Riesselmann. Limitations of a Standard Model Higgs Boson. arXiv:hep-ph/9711456v1, September 1997.

[9] LEPEW Working Group http://lepewwg.web.cern.ch/LEPEWWG/. 
[10] L3 ALEPH, DELPHI and OPAL Collaborations: G. Abbiendi et al. Search for the Standard Model Higgs Boson at LEP. Phys. Lett. B, (565):61-75, 2003.

[11] The TEVNPH Working Group. Combined CDF and DØ Upper Limits on Standard Model Higgs-Boson Production with up to $4.2 \mathrm{fb}^{-1}$ of Data. FERMILAB-PUB-09-060-E, March 2009.

[12] TeV4LHC Working Group http://maltoni.home.cern.ch/maltoni/TeV4LHC/.

[13] A. Djouadi et al. HDECAY: a Program for Higgs Boson Decays in the Standard Model and its Supersymmetric Extension. Comput. Phys. Commun., (108):56-74, 1998.

[14] The CDF Collaboration: T. Aaltonen et al. Search for the Higgs boson in events with missing transverse energy and $b$ quark jets produced in proton-antiproton collisions at $\sqrt{s}=1.96 \mathrm{TeV}$. Phys. Rev. Lett., (100):211801, 2008.

[15] The D0 Collaboration: V.M. Abazov et al. Search for the Standard Model Higgs Boson in the $Z H \rightarrow \nu \bar{\nu} b \bar{b}$ Channel. Phys. Rev. Lett., (97):161803, 2006.

[16] Fermi National Accelerator Laboratory (Fermilab) http://www.fnal.gov.

[17] Fermilab Beams Division. Collider Run II Luminosity http://www-bdnew.fnal.gov/operations/lum/lum.html.

[18] The D0 Collaboration: V.M. Abazov et al. The Upgraded D0 Detector. Nucl. Instr. Meth., A(565):463-537, 2006.

[19] The D0 Collaboration: V.M. Abazov et al. The Muon System of the Run II D0 Detector. Nucl. Instr. Meth., A(552):372-398, 2005.

[20] T. Andeen et al. The D0 Experiment's Integrated Luminosity for Tevatron Run IIa. Technical Report 2365, Fermilab, 2007. 
[21] C. Amsler et al. Particle data group. Phys. Lett. B, 667(1), 2008.

[22] S. L. Robinson. Neutral Supersymmetric Higgs Boson Searches at D0. PhD thesis, Imperial College London, 2008.

[23] T. Scanlon. b-Tagging and the Search for Neutral Supersymmetric Higgs Bosons at D0. PhD thesis, Imperial College London, 2006. FERMILABTHESIS-2006-43.

[24] R. Illingworth. Raw Data Format and Unpacking for the SMT. D0 Note 3829, February 2001.

[25] D. Bauer and R. Illingworth. Level 3 Trigger: Clustering in the SMT. D0 Note 3822, January 2001.

[26] T. Scanlon. Improvements to the Level 3 SMT Unpacking Tool and Tracking in p16.04.00. D0 Note 5372, August 2007.

[27] R. Beuselinck. The RunIIb Level 3 Tracking Tool. D0 Note 5452, July 2007.

[28] C. Barnes et al. Vertexing for the Level-3 Trigger. D0 Note 4271, July 2005.

[29] V. Buscher. Calorimeter Clustering Tool for D0 Level 3 Trigger at RunII http://www-d0.fnal.gov/computing/algorithms/level3/jets/13fcalcluster.ps.

[30] T. Christoudias and P. Jonsson. Certification of the Level-3 Impact Parameter b-tagger. D0 Note 5513.

[31] DØ Trigger Simulator (TrigSim) http://www-d0.fnal.gov/computing/trigsim/trigsim.html.

[32] T. Sjostrand et al. Pythia 6.3 physics and manual. 2003.

[33] A. Zabi et al. A Trigger For Jets and Missing $E_{T}$. D0 Note 4315.

[34] M. L. Mangano et al. ALPGEN, a generator for hard multiparton processes in hadronic collisions. JHEP, 07:001, 2003. 
[35] J. Campbell et al. MCFM - Monte Carlo for FeMtobarn processes http://mcfm.fnal.gov.

[36] M. Begel et al. Determination of Weighting Factors for ALPGEN Monte Carlo Signal and Background Samples. D0 Note 5016, April 2006.

[37] R. Brun and F. Carminati. Program Library Long Writeup. CERN, 1993.

[38] A. Haas et al. D0 Level 1 and Level 2 Calorimeter Trigger Performance in Multi-Jet Events. D0 Note 4073.

[39] F. Couderc et al. D0 Search for Neutral Higgs Bosons at High $\tan \beta$ in Multijet Events Using p17 Data. D0 Note 5202.

[40] S. Mansoora. Search for Pair Production of Scalar Top Quarks in Jets and Missing Transverse Energy Channel with the Do Detector. PhD thesis, Kansas State University, 2008.

[41] The D0 Collaboration. Search for Third Generation Leptoquarks and Scalar Bottom Quarks in $p \bar{p}$ collisions at $\sqrt{s}=1.96 \mathrm{TeV}$. D0 Note 5931-CONF.

[42] The D0 Collaboration. Search for the standard model Higgs boson in the $Z H \rightarrow \nu \bar{\nu} b \bar{b}$ channel in $2.1 \mathrm{fb}^{-1}$ of $p \bar{p}$ collisions at $\sqrt{s}=1.96 \mathrm{TeV}$. D0 Note 5586-CONF, February 2008.

[43] The D0 Collaboration: V.M. Abazov et al. Measurement of the inclusive jet cross-section in $p \bar{p}$ collisions at $s^{1 / 2}=1.96 \mathrm{TeV}$. Phys. Rev. Lett., 101:062001, 2008.

[44] U. Bassler et al. Towards a Coherent Treatment of Calorimetric Energies. D0 Note 4124, March 2002.

[45] N. Makovec and J-F. Grivaz. Shifting, Smearing and Removing Simulated Jets. D0 Note 4914, November 2005. 
[46] J. Hays et al. Electron Likelihood Efficiency in p17. D0 Note 5114, September 2006.

[47] P. Calfayan et al. Muon Identification Certification for p17 data. D0 Note 5157, february 2007.

[48] A. Haas. D0 Search for $Z H \rightarrow \mu^{+} \mu^{-} b \bar{b}$ using p17 Data with NN Optimization. D0 Note, July 2007.

[49] S. Fu. A search for $Z H \rightarrow e^{+} e^{-} b \bar{b}$ using p17 Data with NN Optimization. D0 Note, June 2007.

[50] M. Anastasoaie et al. Search for $W H$ Production with $1 \mathrm{fb}^{-1}$ of RunIIa Data. D0 Note 5356, March 2007.

[51] The D0 Collaboration. Combined Upper Limits of SM Higgs Production from the D0 Experiment. D0 Note 5487, August 2007.

[52] The D0 Collaboration. Combined Upper Limits of SM Higgs Production from the D0 Experiment. D0 Note 5504, December 2007.

[53] M. Tomoto. Search for the Standard Model Higgs Boson in the $Z H \rightarrow$ $\nu \bar{\nu} b \bar{b}$ Channel. D0 Note 4744, April 2005.

[54] T. Scanlon. A Neural Network b-tagging Tool. D0 Note 4889, August 2005.

[55] M. Anastasoaie et al. Performance of the NN b-tagging Tool on p17 Data. D0 Note 5213, January 2007.

[56] ROOT TMultiLayerPerceptron http://root. cern.ch/root/html/TMultiLayerPerceptron.html.

[57] K. Hornik et al. Multilayer Feedforward Networks are Universal Approximators. Neural Networks, 2:359-366, 1989.

[58] D. W. Ruck et al. The Multilayer Perceptron as an Approximation to a Bayes Optimal Discriminant Function. IEEE Transactions on Neural Networks, 1(4):296-298, 1990. 
[59] C. Delaere. TMultiLayerPerceptron: Designing and using Multi-Layer Perceptrons with ROOT.

[60] R. Fletcher. Practical Methods of Optimization. Wiley, 2nd edition, 1987.

[61] T. Andeen et al. FERMILAB-TM-2365. Technical report, Fermilab, 2007.

[62] G. Bernardi et al. Cross Section Errors Used in the Higgs Searches. D0 Note 5043, April 2006.

[63] T. Nunnemann. MCFM and Alpgen Studies on $W b \bar{b}$. D $\varnothing$ Simulation Meeting, September 2006.

[64] T. Junk. Confidence Level Computation for Combining Searches with Small Statistics. Nucl. Instrum. Meth., A434:435-443, 1999.

[65] W. Fisher. FERMILAB-TM-2386-E. Technical report, Fermilab, 2007.

[66] M. Abolins et al. Design and Implementation of the New D0 Level-1 Calorimeter Trigger. Nucl. Instrum. Methods in Phys. Res., $\mathrm{A}(584 / 1): 75,2007$.

[67] J. Pumplin et al. New Generation of Parton Distributions with Uncertainties from Global QCD Analysis. JHEP, 0207(012), 2002.

[68] D. Stump et al. Inclusive Jet Production, Parton Distributions, and the Search for New Physics. JHEP, 0310(046), 2003.

[69] The D0 Collaboration: V.M. Abazov et al. Measurement of the Shape of the Boson-Transverse Momentum Distribution in $p \bar{p} \rightarrow Z / \gamma^{*} \rightarrow e^{+} e^{-}+$ $X$ Events Produced at $\sqrt{s}=1.96 \mathrm{TeV}$.

[70] K. Melnikov and F. Petriello. Electroweak Gauge Boson Production at Hadron Colliders through $\mathcal{O}\left(\alpha_{s}^{2}\right)$. Phys. Rev. D, 74(114017), 2006.

[71] CompHEP collaboration. CompHEP - Automatic Computations from Lagrangians to Events. Nucl. Instr. Meth., A534:250, 2004. 
[72] M. Cacciari et al. The $t \bar{t}$ cross-section at $1.8-\mathrm{TeV}$ and 1.96-TeV: A study of the systematics due to parton densities and scale dependence.

[73] N. Kidonakis and R. Vogt. Next-to-next-to-leading order soft-gluon corrections in top quark hadroproduction.

[74] N. Kidonakis. Single top quark production at the Fermilab Tevatron: Threshold resummation and finite-order soft gluon corrections.

[75] S. Catani et al. Soft-gluon resummation for Higgs boson production at hadron colliders. JHEP, 307, 2003.

[76] E. Busato et al. Jet Algorithms in the DØ RunII Software. D0 Note 4457, August 2004.

[77] A. Harel. Jet ID Optimization. D0 Note 4919.

[78] J. Kvita and K. Peters. RunII $\phi$-Intercalibration of the Fine Hadronic Calorimeter. D0 Note 5005.

[79] B. Knuteson et al. The Missing Transverse Energy Resolution of an Event. D0 Note 3629, June 2000.

[80] A. Schwartzman. Missing $E_{T}$ Significance Algorithm in RunII Data. D0 Note 4253, September 2003.

[81] A. Schwartzman. $\#_{T}$ Significance Algorithm: User's Guide. D0 Note 4253, July 2003.

[82] The D0 Collaboration. Search for neutral Higgs boson in multi-b-jet events in $p \bar{p}$ collisions at $\sqrt{s}=1.96 \mathrm{TeV}$. D0 Note 5726-CONF, July 2008.

[83] The TEVNPH Working Group. Combined CDF and D U Upper Limits on Standard Model Higgs-Boson Production. D0 Note 5536, December 2007. 